\title{
The Institute of Paper Chemistry
}

Appleton, Wisconsin

\section{Doctor's Dissertation}

\section{Anodic Reactions of Simple Phenolic Compounds}

Frederick J. Vermillion, Jr.

June, 1963 
ANODIC REACTIONS OF SIMPLE PHENOLIC COMPOUNDS

A thesis submitted by

Frederick J. Vermillion, Jr.

B.S. 1957, University of Maine

M.S. 1958, University of Maine

M.S. 1960, Lawrence College

in partial fulfillment of the requirements

of The Institute of Paper Chemistry

for the degree of Doctor of Philosophy

from Lawrence College,

Appleton, Wisconsin

June, 1963 
Previous Electrochemical Studies of Phenols

Acetonitrile as a Solvent for Electrochemical Studies

Equipment

Voltammetry

Electrolysis

Millicoulometry

Chemicals

Acetonitrile

Supporting Electrolyte 22

Additives for Voltammetric Studies

Phenols and Other Electroactive Compounds

Electrode Pretreatment for Voltammetry

Voltammetric Procedure

Electrolysis of 2,6-Di-tert-butyl-p-cresol

Electrolysis of the Vanillinate Anion

VOLTAMMETRY: THEORY, AND ANALYSIS OF DATA

PHENOXONIUM ION MECHANISM IN ACETONITRILE

Detailed Studies on 2,6-Di-tert-butyl-p-cresol

Effect of Concentration

44

Effect of Hydrogen Ion Concentration

Buffered Solutions

Effect of Water and Methanol 
Discussion 67

$\begin{array}{ll}\text { Other Hindered Phenols } & 70\end{array}$

Correlation of Half-Wave Potentials $\quad 72$

Mechanism of the Electrode Reaction $\quad 79$

FREE RADICAL MECHANISM OF PHENOXIDE ANIONS IN ACETONITRILE 81

$\begin{array}{ll}\text { Hindered Phenols } & 81\end{array}$

$\begin{array}{ll}\text { Vanillinate Anion } & 83\end{array}$

Voltammetry

Electrolysis 93

Correlation of Half-Wave Potentials $\quad 95$

$\begin{array}{ll}\text { Mechanism of the Electrode Reaction } & 97\end{array}$

AQUEOUS BUFFERED SOLUTIONS 99

2,6-Di-tert-butyl-p-cresol $\quad 102$

$\begin{array}{ll}\text { Vanillin } & 106\end{array}$

$\begin{array}{ll}\text { SUMMARY } & 110\end{array}$

$\begin{array}{ll}\text { ACKINOWLEDGMEIVTS } & 113\end{array}$

$\begin{array}{lr}\text { LITERATURE CITED } & 114\end{array}$

$\begin{array}{lr}\text { APPENDIX } & 118\end{array}$

Further Experiments With Phenols in Acetonitrile $\quad 118$

2,6-Dimethoxyphenol 118

$\begin{array}{lll}\text { Is oeugenol } & 119\end{array}$

$\begin{array}{ll}\text { Effect of Pyridine } & 120\end{array}$

The Use of Hammett Correlations With Half-Wave Potentials 125

Totally Irreversible Electrode Reaction - 125

Reversible Reaction $\quad 126$

Reversible Reaction of Phenols, Buffered Media 126 
Because of the common occurrence and extreme reactivity of the phenolic hydroxyl group it has been the object of special study in the field of wood chemistry. Phenolic groups are found in extractives, lignin precursors, protolignin, and lignin degradation products. The oxidation of phenols is particularly important in the biosynthesis of lignin, in studies on isolated lignins, and in commercial pulping and bleaching operations.

Electrochemical oxidation has been virtually unexplored by previous workers in the field of lignin chemistry. Brauns $(\underline{1}, \underline{2})$ has reviewed the few studies which have been conducted at uncontrolled potential. O'Connor (3) has recently completed work on the voltammetry and controlled potential oxidation of 3,4-dimethoxypropenylbenzene.

Electrochemical methods afford advantages over more conventional chemical techniques in studying the reaction mechanisms of organic compounds. Voltammetry and related methods give simple direct evidence for the reactive intermediate in an electrochemical oxidation or reduction. Thus, the effects of solvent, pH, etc., can be studied apart from the complicating effects of specific chemical oxidants. The potential at which an electrochemical reaction occurs is a direct quantitative measure of molecular reactivity. It should, therefore, be possible to develop correlations from electrochemical studies which are also valid for conventional chemical methods. Eventually such correlations should lead to a better basic understanding of the reactions which are important in wood chemistry, and this in turn will provide the means for more efficient chemical processing of wood.

The present study was undertaken with the objective of elucidating the anodic reaction mechanisms of simple phenolic compounds using the techniques of voltammetry, 
coulometry, and controlled potential electrolysis. The majority of the experimental work has been conducted in acetonitrile solutions because of the wide potential range available for anodic study. Limited voltammetric studies in aqueous buffered solutions have shown that the conclusions can be extended to other media. The results in acetonitrile have been obtained using platinum electrodes, while those in aqueous systems have made use of both platinum and graphite electrodes. 


\section{LITERATURE REVIEW}

\section{PREVIOUS ELECTROCHEMICAL STUDIES OF PHENOIS}

In electrochemical oxidations of phenolic compounds, at uncontrolled potential, small yields of dimeric products, polyhydroxylated compounds, and quinones have been obtained (4). These results were generally interpreted in terms of a free radical mechanism. Electrolyses were usually complicated by the formation of tarlike side products. Anodic substitution of phenolic compounds in ortho and para positions with thiocyanate has been reported in acidic solutions, in yields from 50 to $85 \%(\underline{4}, \underline{5})$.

Voltammetric studies of phenolic compounds have been complicated by the formation of polymeric films on the electrode surface. These films cause peaked waves and decreased diffusion currents at higher levels of concentration, and inability to retrace the voltammogram without cleaning the electrode even at a very low level. of concentration. They may be removed from platinum electrodes by burning in the flame of a Bunsen burner ( $\underline{6}, \underline{7}$ ) or by treatment with strong chromic acid cleaning solution (ㅇ, 9 ). Reproducible results are possible only after such pretreatment.

Voltammetric results on platinum electrodes are usually affected by the oxidation state of the electrode surface. With the phenols, three authors ( $1-\underline{9}$ ) have indicated that more satisfactory results were obtained on an oxidized surface and Ginzberg ( $I$ ) believes that the surface oxides of platinum actually participate in the electrode reaction.

The carbon electrode has been used for a number of voltammetric studies of phenolic compounds because its surface can be easily renewed, and it is free from the effects of oxide films. Apparently, it gives approximately the same half-wave 
potentials as the platinum electrode ( $\underline{7}, \underline{10})$. The boron carbide electrode has been investigated briefly with $\alpha$, and $\beta$-naphthol, and likewise gives approximately the same half-wave potentials as are obtained with platinum (그). The problem of polymeric films still exists with either carbon or boron carbide electrodes (프).

Hedenberg and Freiser (6). studied the mechanism of oxidation of phenol; and the monosubstituted tertiary butyl phenols in aqueous solutions in the $\mathrm{pH}$ range from 5 to 11 , using a rotating platinum anode. They were able to obtain normal waves only at concentration levels of $8 \times 10^{-5} \underline{M}$ or lower. On the basis of logarithmic plots of $\underline{i} /\left(\underline{i}_{\underline{D}}-\underline{i}\right)$ vs. $\underline{E}$ these authors interpreted their results as indicating a one-electron reversible reaction with a phenoxy free radical as the product of the primary electrode process.* Two factors led them to believe that the electroactive species was the phenoxide anion rather than the nonionized phenolic'molecule. First, only one wave was observed, and the wave height was proportional to the total concentration of phenol. Second, the half-wave potential showed little $\mathrm{pH}$ dependence when the $\mathrm{pH}$ was greater than $\underline{\mathrm{pK}}_{\mathrm{D}}$ Ginzberg (7) postulated essentially the same mechanism as Hedenberg and Freiser except that he believed that the oxide film on the platinum electrode participated in the electrode reaction.

Gaylor, et al., (13, 14) studied the voltammetry of various phenols and hydroquinone in an isopropanol water mixture at the wax-impregnated graphite electrode over the $\mathrm{pH}$ range from 1.2 to 10.0 . By a comparison of the diffusion current constants of the phenols with the diffusion current constant of hydroquinone they reached the admittedly tentative conclusion that the main electrode reaction of the phenols involved a two-electron transfer. At pH 1.2 a doubling 
of the diffusion current of the phenolic compounds was noted, and this was presumed to be a four-electron process. These conclusions were based on the assumption that hydroquinone was oxidized in a two-electron reaction. The main basis for such an assumption was the observation that quinhydrone gave a single smooth wave at the graphite electrode. However, in more recent work Elving and Krivis (15), and Morris and Schempf (16) have independently reported that quinhydrone gives two waves on the graphite electrode. The latter authors interpret the anodic wave of hydroquinone on a' graphite electrode as a one-electron reversible process from logarithmic plots of $\underline{i} /\left(\underline{i}_{\underline{D}}-\underline{i}\right)$ vs. potential. If this is the case, a one-electron reaction would be indicated for phenolics from the data of Gaylor, et al. (13, 14) except at a very low level of $\mathrm{pH}$ where a two-electron reaction would be indicated. This would then agree with the conclusions of Hedenberg and Freiser (ㅁ) . However, the results of Nash, Skauen, and Purdy (17) reveal variations between different phenolic compounds which would seem to preclude the possibility of a single, simple mechanism.

Several investigations have been concerned with the relative half-wave potentials of different phenols because it has been found that the half-wave potential can be correlated with antioxidant efficiency (17-19). The work of Penketh (18) and Suatoni, Snyder, and Clark (20) has demonstrated that the effect of ortho, meta, and para substituents on the half-wave potential of phenols is generally additive. The latter authors were able to calculate half-wave potentials for disubstituted phenols within 0.01 volt from the values of half-wave potentials for monosubstituted phenols, except in the cases of the 2,6-disubstituted compounds and dichloro compounds. They also demonstrated a correlation within 0.08 volt for $E_{1 / 2}$ vs. the Hammett $\sigma$ function (I) for the meta- and para-substituted phenols. 
In chronopotentiometric studies, Elving and Krivis (르) have investigated the behavior of the isomeric dihydroxybenzenes at the wax-impregnated graphite electrode; Adams, McClure, and Morris (ㅇ) have investigated the substituted naphthols at a platinum electrode; and Voories (9) has briefly investigated $\alpha$ - and $\beta$-naphthol at a platinum anode. The latter author reports that the apparent $\underline{n}$ value for $\beta$ naphthol changed from 1 to 2 on going from a borate buffer ( $\mathrm{pH}$ 9.2) to a phosphate buffer ( $\mathrm{pH}$ 1.2.2).

Voltammetric data on 2,6-di-tert-butyl-p-cresol, a compound of particular interest in the present study, has been obtained by Penketh (18) who reports that this phenol and 6-tert-butyl-4-methylphenol were the only two which he observed to give distinct and reproducible waves on both platinum and graphite electrodes. He points out though that the slope of this wave was very gradual and that the diffusion current was ill-defined. Barendrecht (23), using graphite electrodes, observed a similar result in acidic solutions, but was able to obtain a welldefined, evidently reversible wave in a basic solution.

The only report on the anodic voltammetry of vanillin, another compound of special interest in the present investigation, was contained in the paper by Nash, Skauen, and Purdy (17). A half-wave potential of 0.508 volti, and an $\underline{n}$ value of 1.03 from logarithmic analysis was reported on the wax-impregnated graphite electrode at a pH of 7.0. Pearl ( 24 ) has obtained a small yield of vanillil and larger quantities of vanillic acid from an electrolysis of vanillin at uncontrolled potential on a mercury anode in basic solution.

There are no published papers on the voltammetry of phenols in acetonitrile, but. Iund (25) in unpublished work has observed deformed waves for certain phenols in this medium. He reports that upon addition of pyridine the single wave is split 
into two waves. Geske (26) evidently is conducting a study of phenoxide anions in acetonitrile, particularly the picrate anion (그).

\section{ACETONITRILE AS A SOLVENT FOR ELECTROCHEMICAL STUDIES}

Acetonitrile has been a popular solvent for voltammetric investigations in recent years mainly because of the wide range of potential between its anodic and cathodic decomposition limits. The papers of Geske (26, 28-30) and of Iund (31, 32) have been specifically concerned with anodic voltammetry of organic compounds, and adequately review the previous literature. The papers of Kolthoff, et al. (33-36) are especially recommended because they give a clear description of the properties peculiar to acetonitrile which are responsible for the observed voltammetric results with this solvent. These may be stated briefly as follows:

1. The relatively high dielectric constant $(\epsilon=38)$ promotes the dissociation of strong electrolytes such as the metallic perchlorates.

2. Cations have a very low solvation energy in acetonitrile, i.e., acetonitrile is a weak Lewis base.

3. Anions have extremely low solvation energies in acetonitrile, i.e., acetonitrile is an even weaker Lewis acid than it is a base.

4. Acetonitrile is a relatively good solvent for many organic species.

The first of these properties is important because it is desirable to conduct voltanmetric investigations in the presence of a large concentration of indifferent supporting electrolyte so that effects of electrical migration currents will be minimized (37). The fourth property has obvious importance for the electrochemical study of organic compounds.

The second and third properties together with the first are responsible for the behavior of acids and bases in acetonitrile. Thus, it has been found that, of the common acids, perchloric acid is the only one which exhibits typical strong 
acid behavior in this solvent (36). The other acids which are considered to be strong acids in water have $\underline{\mathrm{pK}_{\mathrm{D}}}$ values ranging from 5.5 to 8.9 in acetonitrile. Furthermore, except in very dilute solution they dissociate according to the equation $2 \mathrm{HA} \rightleftarrows \mathrm{H}^{+}+\mathrm{AHA}^{-}$instead of $\mathrm{HA} \rightleftarrows \mathrm{H}^{+}+\mathrm{A}^{-}$; in other words, the anion is solvated by a molecule of undissociated acid.

Acetic acid is so weak in acetonitrile that its dissociation can for practical purposes be neglected. The phenols then must be regarded as practically nonionized in acetonitrile except in the presence of added base. This conclusion is very significant in view of the results obtained in the present study. 


\section{EXPERIMENTAL}

\section{EQUIPMENT}

\section{VOLTAMMETRY}

A Sargent Model'XII polarograph was used in this investigation. After a few experiments it was found that the photographic recording feature of this instrument was completely unsuitable for the type of experimental work contemplated, and the instrument was converted to chart recording as suggested by Licht, et al. (38). A block diagram of the circuit is presented in Fig. 1. The Sargent recording potentiometer, on a suitable $\mu$ a. range was connected into the circuit in series with the cell. The galvanometer shunt ratio switch of the polarograph was set at 1000 at all times so that the galvanometer would not be damaged. The voltage zero shifting circuit within the dotted lines could be used to displace the zero point of the voltammogram by placing a known e.m.f. across the segment ab of the resistor, $R$, in series with the cell. By suitable positioning of the switch, $S_{2}$, displacement of the zero point in either the positive or negative direction could be achieved. The battery, B, was of the lead storage type, so selected because it had a negligibly low internal resistance. By positioning switch $S_{1}$ to the $c$ position and $S_{3}$ to the off position, the voltage zero shifting element of the diagram is eliminated from the circuit. The full span potential of the polarograph could be conveniently measured with the student potentiometer by positioning of switch, $\mathrm{S}_{4}$, to the a side. When switch, $\mathrm{S}_{4}$, is positioned to the e side the zero shift voltage could be measured. The switch, $S_{4}$, was kept in the off position when running a voltammogram. The cell leads were disconnected when voltages were being measured with the student potentiometer. 


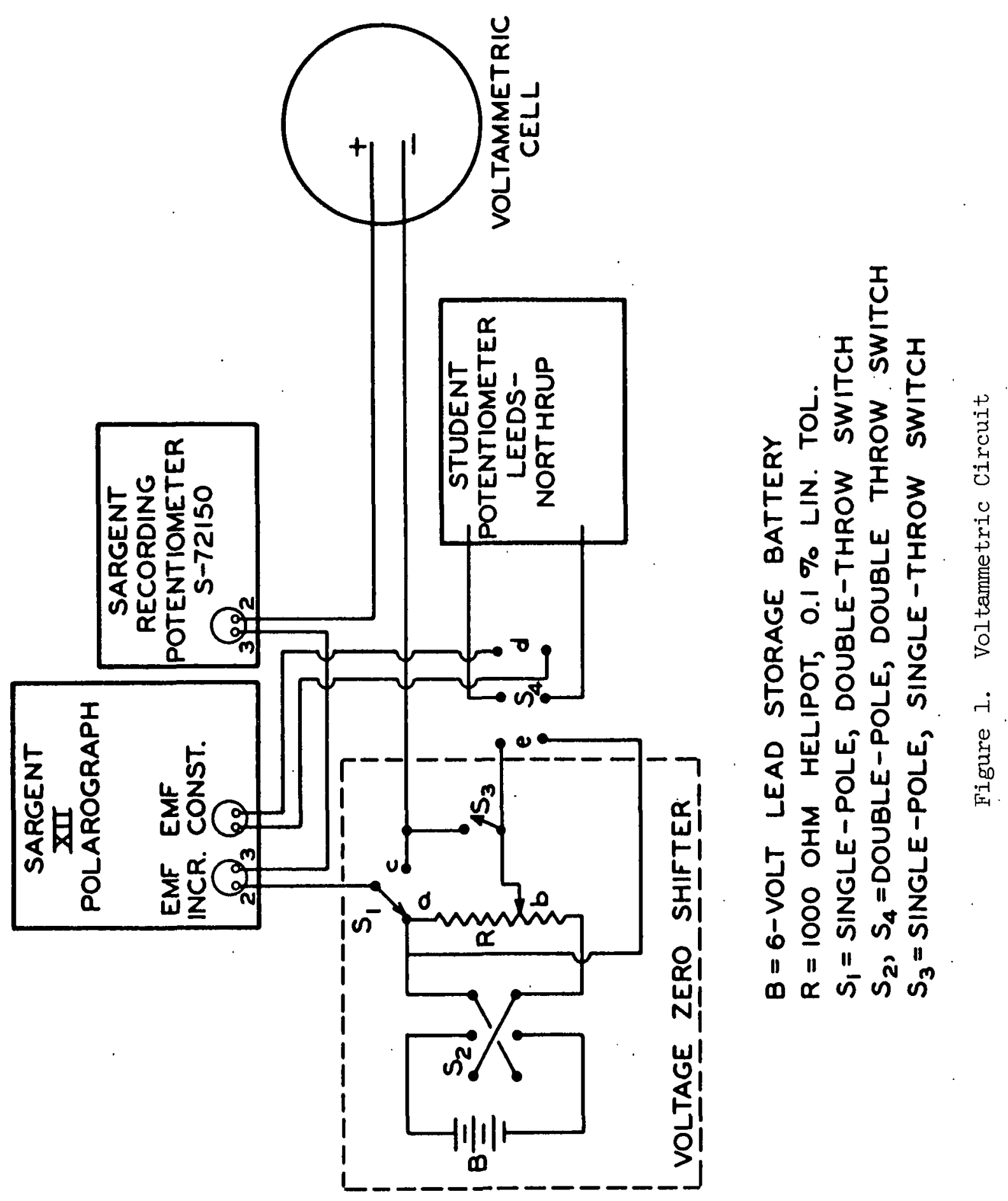


In this circuit two resistances are placed in series with the cell, and these must be added to the measured cell resistance in order to calculate the proper $\underline{i R}$ arop for correction of recorded voltammograms. The first of these is a 1000-ohm fixed resistance within the Sargent recorder, and the second occurs in the voltage zero shifting circuit. This second resistance depends on the setting of the helipot, $\underline{R}$, and may be calculated by the familiar formula for parallel resistances, $\underline{R}_{e f}=$ $I / \underline{R}_{1}+I /\left(1000-\underline{R}_{1}\right)$ where $\underline{R}_{\text {eff }}$ is the effective resistance placed in series with the cell, and $\underline{R}_{1}$ is the resistance setting of the $1000 \Omega$ helipot.

In the wiring of the voltammetric circuit care was taken to use grounded, shielded cable for all connections. All auxiliary electrical equipment and the water bath control and heating elements were individually grounded. The Sargent synchronous rotator which was used for rotating the working electrodes was equipped with a grounded shielded cable. Despite these precautions some stray electrical disturbances which resulted in excessive chatter of the recorder pen were occasionally noted and this made voltammograms on the lower concentration levels of the electroactive species $\left(<5 \times 10^{-5} \underline{\mathrm{M}}\right.$ ) difficult to interpret. This effect seemed to be more pronounced in the winter months when the relative humidity was lowest.

It is recommended that for future work consideration be given to an improved voltammetric circuit. Equipment should be chosen with two objectives in mind. First, it would be very desirable to be able to work at lower levels of concentration of the electroactive species. Second, it would be a decided advantage to have equipment which would give voltammetric curves which are free from îR distortion. A discussion of suitable polarographic circuits is given in the recent review by Hume (39).

In the first experiments, a Leeds \& Northrup conductivity bridge was used for resistance measurements. It was later found that the measured resistance depended 
upon the scale setting of the bridge, probably because of the low frequency a.c. source (60 c.p.s.). In order to obtain a more reliable value, a General Radio Type 1603-A impedance bridge was used in later experiments with a Hewitt Packard Model 200-B audio oscillator set at 3750 c.p.s. as a signal generator, and a Tektronix Type 502 oscilloscope as a signal detector. The block diagram for this circuit is shown in Fig. 2. It was found that increasing the frequency of the source above 3750 c.p.s. had no effect upon the measured value of resistance with this equipment. The sensitivity of this equipment was high enough so that the voltage output from the audio oscillator could be maintained at a value of less than 0.1 volt. Increasing the voltage output had no effect on the measured resisance.

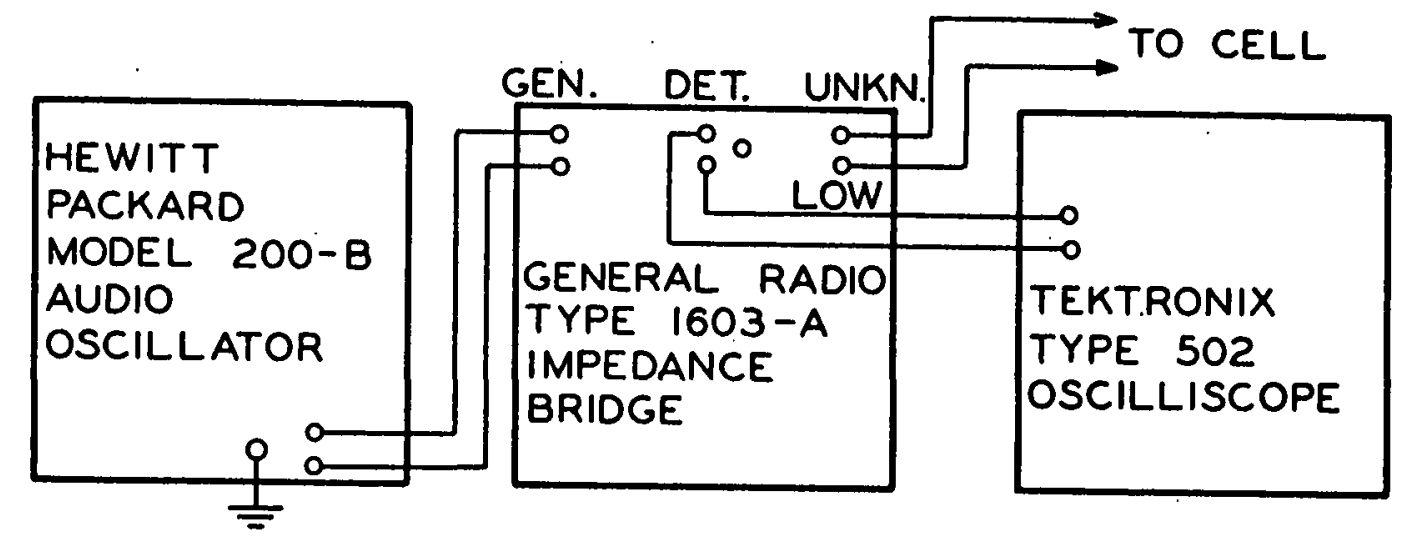

Figure 2. Resistance Measuring Circuit

(The leads to the cell were shielded cable with the shield grounded to the "LOW" side of the bridge circuit.)

Some of the data reported in a later section of this thesis were obtained before the improved resistance bridge was in use. The maximum error which could have been introduced into the half-wave potential for a diffusion current constant of $40 \mu \mathrm{a} . / \mathrm{mM}$ is estimated for concentration levels of $1,0.5$, and $0.1 \mathrm{mM}$ to be -0.05 , -0.025 , and -0.005 volt, respectively. This error will not affect the conclusions drawn from the data, and the questionable values are so indicated in the presentation of data. 
The voltammetric cell which was used in this investigation was patterned after the design of Kolthoff and Coetzee (32). This cell permits the use of the aqueous saturated calomel electrode (s.c.e.) with a nonaqueous solution in voltammetric studies. The agar bridge from the reference electrode is immersed in a cylinder containing a small amount of the test solution which was isolated from the sample in the cell with a grade fine frit. The level of the liquid in the cylinder above the frit is maintained below the level in the cell so that contamination of the test solution from water and chloride ion is avoided. A detailed diagram of the cell assembly has been given by J. J. O'Connor (ㄹ)

The calomel half cells used in this investigation were prepared as suggested by Meites (40). When not in use, the tip of the agar bridge was kept immersed in a saturated potassium chloride solution. This procedure kept the agar bridge in good condition for extended periods of time, alleviating the problem of increased cell resistance due to hardening of the agar after prolonged contact with acetonitrile.

Prepurified nitrogen (Matheson) was used for deaeration of test solutions. It was passed through two drying tubes, the first containing magnesium perchlorate, and the second containing phosphorus pentoxide, and then through two presaturation tubes containing the test solution solvent. All connections in the nitrogen line after the presaturating tubes were made of glass. The nitrogen could be directed by means of a two-way stopcock either into a dispersion tube immersed in the test solution for deoxygenation or over the surface of the test solution while recording voltammograms.

The nitrogen presaturating tubes were immersed in a water bath maintained at $25.0 \pm 0.2^{\circ} \mathrm{C}$. The voltammetric cell and the s.c.e. were provided with water jackets through which water from this bath was continuously circulated. 
The rotating electrodes used in this study were driven with a Sargent synchronous rotator rated at 600 r.p.m. Checks of rotational speed with a tachometer indicated an actual speed of 618 r.p.m. The cell assembly and rotator were mounted on a specially constructed heavy duty ring stand to minimize vibrations.

In the design of rotating platinum electrodes for voltammetry it has been noted by Geske (28) that the best results are achieved with tiny electrodes which give very small diffusion currents. Large diffusion currents should be avoided because of the excessive $\underline{i}$ drop and the appreciable decrease in the concentration of the electroactive species during the course of one voltammogram. The electrodes used in this study were constructed from platinum wire sealed coaxially into the end of a soft glass tube so that only a very small piece of the platinum was left exposed. This exposed tip had been previously annealed in an oxygen flame so that its surface was very smooth and polished. The surface areas of the electrodes used in this study were estimated by microscopic investigation and are given in Table I. The smaller electrodes (V-3, and V-4) seemed somewhat superior in performance characteristics. Electrical contact to the electrode was achieved through a mercury column in the glass tube. A platinum wire was used to make the electrical connection between the voltammetric circuit and the mercury column.

\section{TABLE I}

SURFACE AREAS OF PLATINUM ELECTRODES

Electrode

No.

7

8

V-3

V-4
Wire Gage

18

18

24

24
Surface Area,

sq. cm.

0.024

0.029

0.0058

0.0031 
A carbon electrode was fabricated so that the carbon paste of Olsen and Adams (10) could be used with a rotating electrode. The design of this electrode is given in Fig. 3. The carbon paste was contained in a $1 / 8$-inch hole bored in one end of a teflon rod. The other end of the teflon rod was bored to fit the 1/4inch brass rod which made electrical contact. with the carbon paste. The brass rod was drilled through its detachable lower section to facilitate the repacking of the carbon paste. The electrode was insulated from the Sargent synchronous rotator by a nylon sleeve. A small cup at the top of the nylon held mercury for external electrical connection. Contact between the mercury and the brass was achieved with a platinum pin through the bottom of the nylon cup.

The carbon paste was formulated with Acheson Grade No. 38 graphite kindly donated by National Carbon Company, and mineral oil (Nujol). The paste was mixed to a desirable consistency and stored in a stoppered jar. After packing the paste into the tip of the electrode the excess was removed, and the surface was smoothed with a spatula.

\section{ELECTROLYSIS}

The potentiostat manufactured by Analytical Instruments Inc. was used for electrolyses. A Sargent Recording Potentiometer (S-72150) on a current range was used to give a record of current-time curves which could be subsequently integrated for coulometric data. The recorder input lugs were connected through shielded cable to J205 on the rear of the potentiostat, and electrolyses were conducted with the "Integrator" switch on. Contrary to the statement of the manufacturer (4I) it was found that a periodic adjustment of the gain control in the amplifier chassis was necessary in order to get a close degree of potential control under the conditions of the electrolyses. The balance control on the front panel was adjusted as prescribed in the instruction manual before the start of the electrolysis. The 


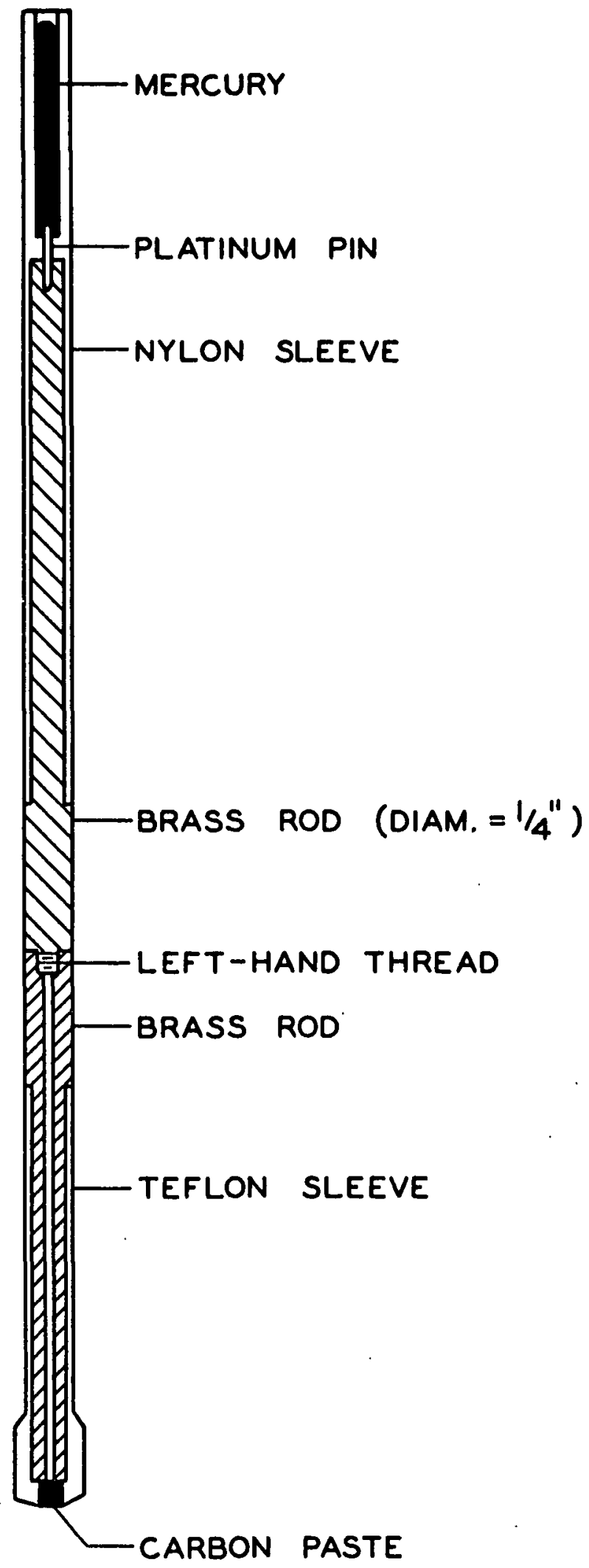

Figure 3. Rotating Carbon Paste Electrode 
gain control was adjusted after the commencement of the electrolysis to the point where the pen of the recorder hunted slightly, at all times. This control had to be readjusted at intervals throughout the electrolysis. By using this procedure the potentiostat gave very satisfactory potential control.

The cell for controlled potential electrolysis consisted of two compartments connected through a side tube with a ball and socket joint so that they could be easily dismantled for cleaning. A grade fine fritted glass was placed in the side tube as close to the anode compartment as possible to prevent bulk flow of solution from one compartment to another. The capacity of the anode compartment was about $600 \mathrm{ml}$. The resistance between the anode and cathode of the assembled cell, using $0.5 \mathrm{M}$ sodium perchlorate as a supporting electrolyte in acetonitrile, was 90 to 100 ohms. The agar bridge from the saturated calomel reference electrode was separated from the bulk of the solution in the anode compartment by a grade fine frit in the same manner as with the polarographic cell. This frit was placed close to the working; electrode so as to minimize any $\underline{i} \underline{R}$ drop in the reference circuit. The working electrode was a rotating cylindrical platinum gauze of 10 sq. cm. area. The auxiliary electrode in the cathode compartment was a quiescent mercury pool. Nitrogen from the same drying and presaturation system as described for the voltammetric assembly was introduced at the bottom of the anode compartment to deaerate the test solution. The anode compartment was immersed in a small water bath with continuous circulation of water from the large bath at $25.0 \pm 0.2^{\circ} \mathrm{C}$. A detailed diagram of the cell assembly has been given by $0^{\prime}$ Connor (므).

For the electrolysis of 2,6-di-tert-butyl-p-cresol, the potentiostat was used to supply a constant current by controlling the voltage drop across a fixed resistor in series with the electrolysis cell ( $96.9 \mathrm{ohms}$ ) as described in the instruction manual of the manufacturer (4I). The Sargent recorder was used for monitoring 
current. During this electrolysis it was necessary to maintain a flow of methanolic tetraethylammonium hydroxide to the cell at a rate proportional to the current flow. This was accomplished in a simple manner with the equipment sketched in Fig. 4. The solution to be added to the cell was stored in the $50-\mathrm{ml}$. buret and flowed through a piece of fine tygon tubing, and then a tiny glass capillary to the anode compartment. The tygon tubing ran through a solenoid valve from a Sargent automatic titrating unit. This solenoid constricted the tygon tube except.when an electrical impulse from the Flexopulse Timer (Eagle Signal Corp.) was received. The timer was adjusted manually at intervals throughout the electrolysis so that the desired rate of addition was maintained. The timing interval between the impulses varied from about thirty-five seconds at the beginning of the electrolysis to twenty seconds toward the end.

This method of controlling the acidity of a nonaqueous solution during an electrolysis is evidently a novel technique in the field of electrochemistry, and should prove of value in future studies. The experimental setup, while satisfactory for the purpose of the present study, could be improved by using an automatic titration device together with a suitable indicator electrode to maintain the "activity" of hydrogen ions constant in the solution. Such an experimental setup would facilitate the carrying out of the electrolysis at controlled potential rather than at controlled current, and would enable more precise control of hydrogen ion "activity" in the solution.

\section{MILIICOULOMETRY}

For millicoulometric determinations the main requirement is that there be an appreciable decrease in the concentration of the electroactive species in a short time interval (42). This means that the cell volume should be small in relation 

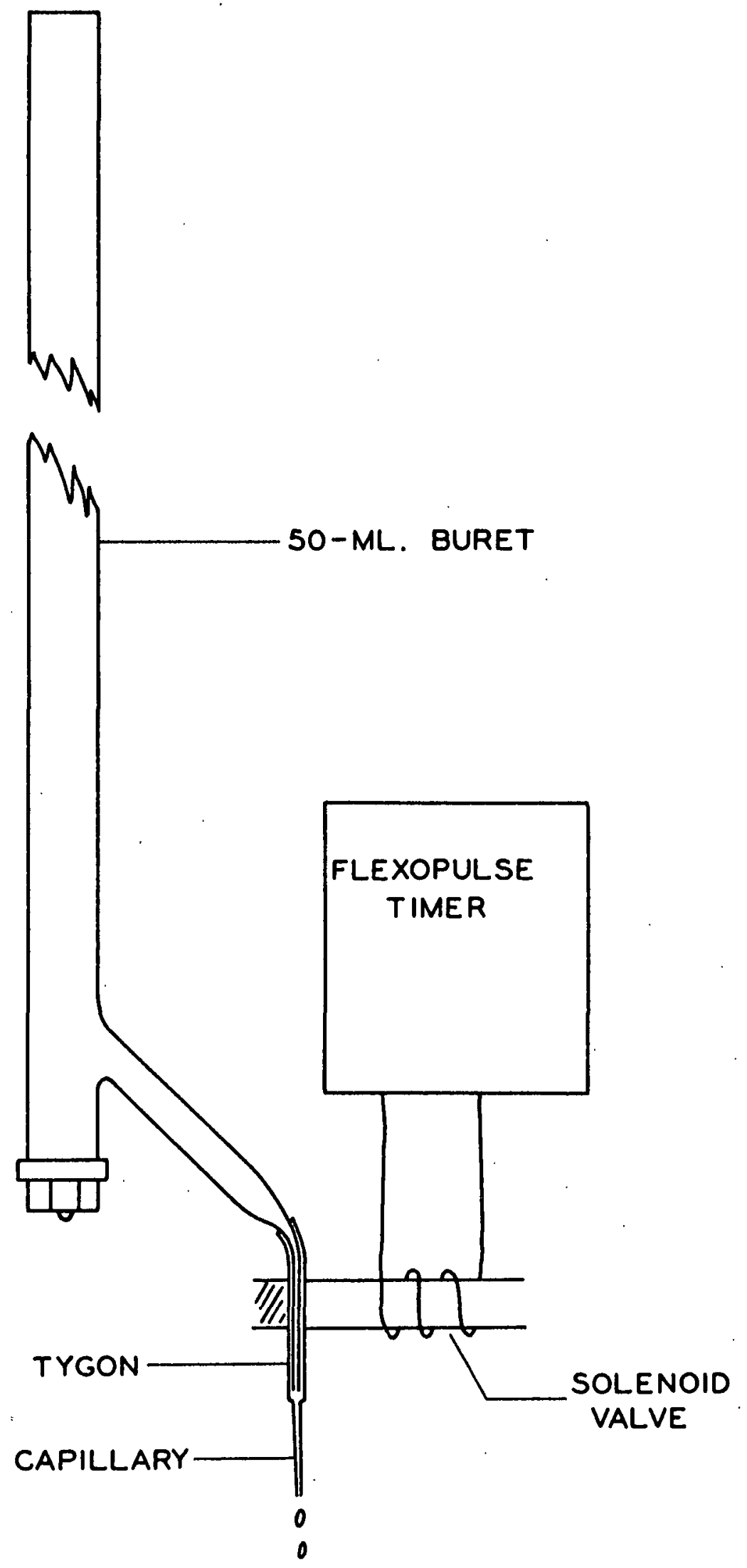

Figure 4. Device for Continuous Addition of Base During Electrolysis 
to the electrode surface area. The cell assembly used in this investigation is diagrammed in Fig. 5.* The volume of liquid used in the working electrode compartment of this cell could range from 0.5 to $3 \mathrm{ml}$. The working electrode, one of the rotating platinum electrodes used for voltammetry, was separated from the reference electrode and the auxiliary electrode by a fine grade frit. The auxiliary electrode was a piece of platinum foil of about one square inch in area (large enough to prevent concentration polarization). Provision was made for deaeration of the test solution through a tiny tube of teflon and glass construction. Bulk flow of solution through the frit was minimized by adjusting the solution to the same level in both arms of the cell. The cefl was enclosed in a water jacket for temperature control.

In order to use the potentiostat with this cell a shunting resistor was placed across the power terminals of the potentiostat as shown in Fig. 6. This maintained a current loading on the instrument which was large enough so that effective potential control was achieved even at very low levels of concentration. The Sargent S-72150 recorder was used during millicoulometric studies for recording current time curves which could be subsequently integrated for calculation of the coulometric $\underline{n}$ value.

\section{CHEMICAIS}

\section{ACETONITRILE}

Acetonitrile was purified by the method of Wawzonek and Runner (43). Attempts to purify the technical-grade product of Union Carbide Company by this method were

\footnotetext{
*This cell was poorly designed because the reference electrode was placed at the same point in the cell as the auxiliary electrode. This places a large iR drop in the control circuit when experiments are conducted at high levels of concentration $\left(>10^{-3} \mathrm{M}\right)$. A better design would place the reference electrode outside of the direct path between the working and auxiliary electrodes.
} 


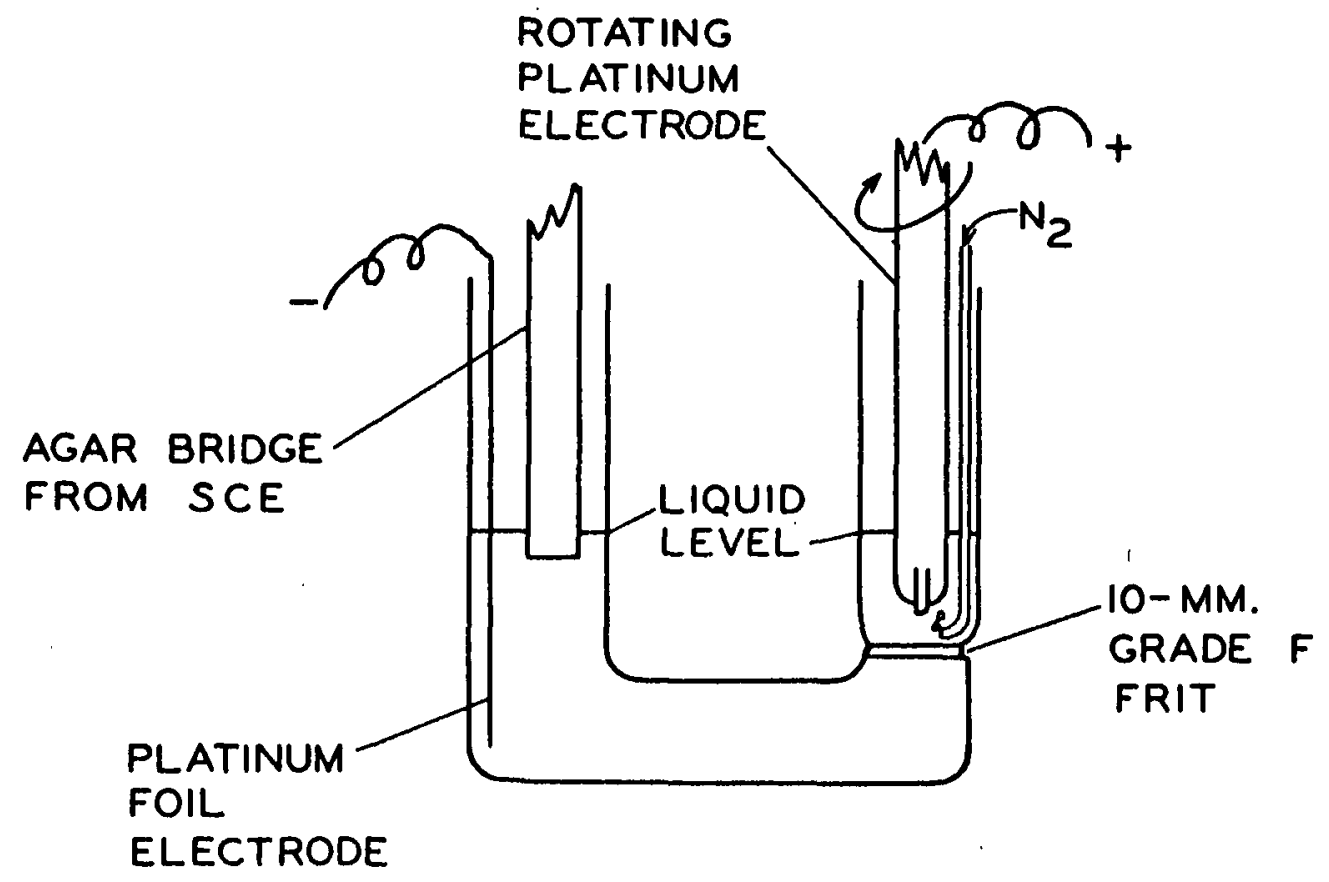

Figure 5. Millicoulometric Cell

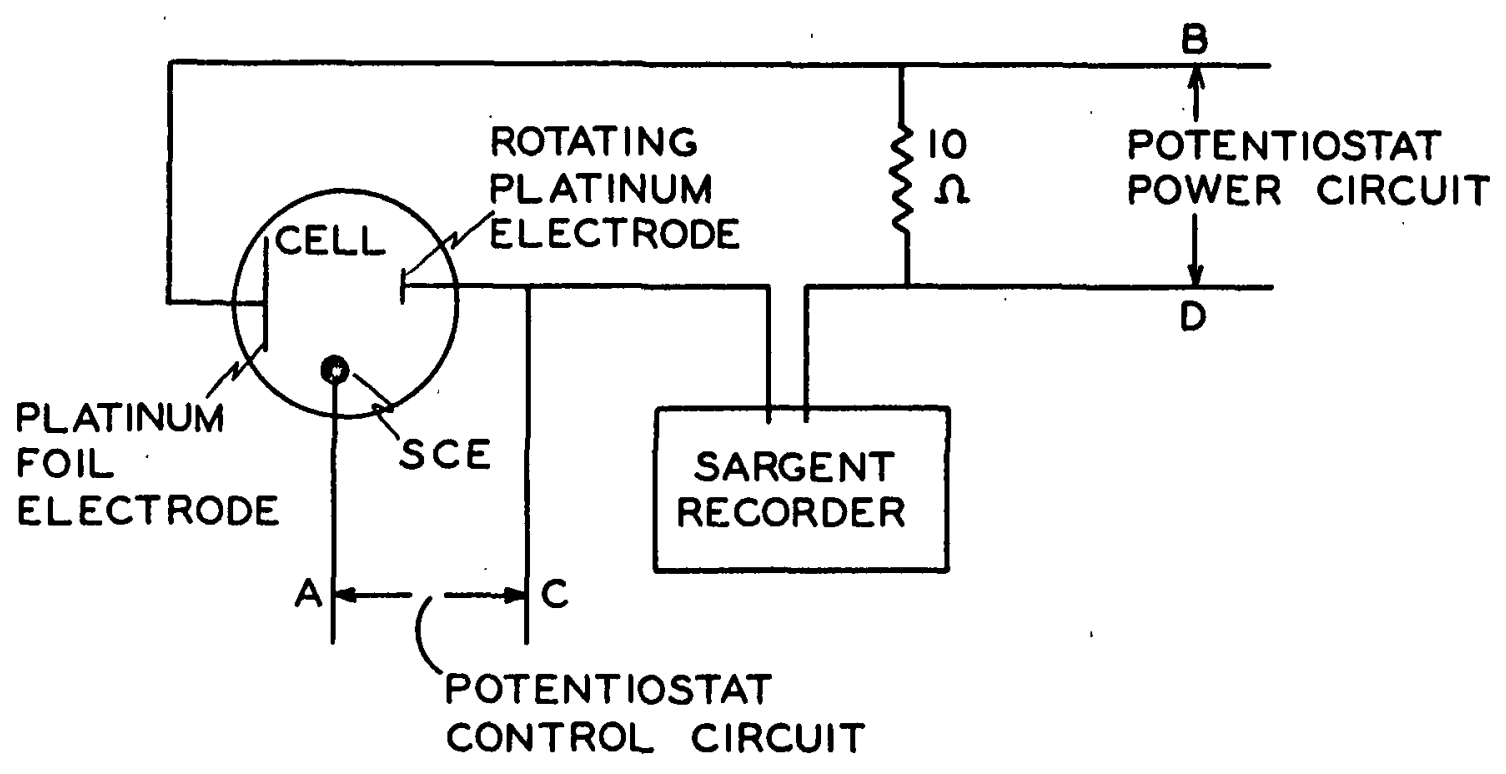

Figure 6. Electrical Circuit for Millicoulometric Cell

(Letters A, B, C, D refer to potentiostat terminals) 
rather unsuccessful, but the practical-grade chemical of Matheson, Coleman, and Bell was completely satisfactory. The acetonitrile in a four-liter bottle was shaken for less than one minute with aqueous saturated potassium hydroxide, stored over anhydrous sodium carbonate for at least twelve hours, and then distilled twice from a small amount of phosphorus pentoxide through a five-foot all glass column packed with glass helices. The column was equipped with a two position, solenoid operated, tilting funnel type distillation head which was operated from a Flexopulse Timer so that precise regulation of the reflux ratio could be achieved. The sides of the column were maintained at the boiling point of the solvent with electrical heating coils. In the first distillation $200 \mathrm{ml}$. of solvent were collected at $95 \%$ reflux and discarded. Then approximately three liters were collected at $25 \%$ reflux and saved for redistillation. The residue of 200 to 300 $\mathrm{ml}$. left in the distillation flask was discarded. In the second distillation 200 $\mathrm{ml}$. collected at $95 \%$ reflux were discarded, approximately $2500 \mathrm{ml}$. were collected for use, and the residue was discarded. The solvent was stored in the collection bottle wrapped in aluminum foil to exclude light. It was displaced from the collection bottle through an all glass siphon with a positive pressure of dry nitrogen. The water content of the acetonitrile thus prepared, after addition of supporting electrolyte, ranged from 1 to $2 \mathrm{mM}$ by Karl Fischer titration.

\section{SUPPORTING ·ELECTROLYTE}

The method of Kolthoff and Coetzee (33) was used to prepare tetraethylammonium perchlorate. It was found that two to three recrystallizations from distilled water were necessary to completely remove the voltammetrically detectable bromide ion from the product. It was dried overnight in a vacuum oven at $80^{\circ} \mathrm{C}$.

Sodium perchlorate (G. F. Smith reagent grade) was dissolved in hot water and filtered to remove the insoluble material. The solution was concentrated on a hot 
plate until precipitation began. It was then cooled until the temperature of the solution reached $70^{\circ} \mathrm{C}$, filtered, and dried in a vacuum oven at $150^{\circ} \mathrm{C}$. overnight.

Most of the voltammetric experiments were conducted with the tetraethylammonium perchlorate as a supporting electrolyte which showed a smaller residual current in solution than the sodium salt, and was more convenient to handle. The electrolyses were conducted using the sodium salt as a supporting electrolyte.

\section{ADDITIVES FOR VOLTAMMETRIC STUDIES}

Pyridine was purified by the method of Burdick, et al. (44). A mixture of spectroquality and reagent-grade pyridine was stored over barium oxide for several days and then distilled from metallic sodium through the column described previously. Starting with about two liters of pyridine, $600 \mathrm{ml}$. were collected at $95 \%$ reflux and discarded, the next $700 \mathrm{ml}$. were collected for use, and the remainder was discarded. The pyridine was stored in the collection bottle which was covered with aluminum foil to keep out light and was equipped with an all glass siphon tube.

Tetraethylammonium hydroxide was used as supplied by Eastman Kodak Company in $10 \%$ aqueous solution. Its titer against a standard $\mathrm{HCl}$ solution was $0.673 \mathrm{M}$.

To prepare a methanolic solution of tetraethylammonium hydroxide, thirty grams of tetraethylammonium bromide (Eastman Kodak) in $100 \mathrm{ml}$. of reagent-grade methanol together with 20 grams of silver oxide were placè on a shaker overnight, filtered through a grade fine frit, decolorized with activated charcoal, filtered again, and diluted to $100 \mathrm{ml}$. with reagent-grade methanol. This solution gave no test for bromide when neutralized with perchloric acid and tested with silver nitrate solution. The solution was titrated against potassium acid phthalate (National Bureau of Standards) in aqueous solution to a phenolphthalein end point and found to be 0.6096 N. 
Perchloric acid was prepared in an anhydrous solution of acetic acid (Baker Analyzed Reagent) by adding sufficient acetic anhydride (Baker Analyzed Reagent). to use up the water in the $70 \%$ acid (G. F. Smith). It was titrated against potassium acid phthalate (National Bureau of Standards) to the methyl violet end point in glacial acetic acid, and found to be 0.519N. Karl Fischer analysis showed a negligible water content.

Tetraethylammonium nitrate was prepared from tetraethylammonium bromide (Eastman Kodak) and silver nitrate (reagent grade). The tetraethylammonium bromide (105 g.) was dissolved in $800 \mathrm{ml}$. of distilled water, and heated almost to the boiling point. Then the silver nitrate was added slowly with mechanical. stirring to achieve good flocculation until no more precipitate formed with further dropwise addition. The solution was cooled, filtered, decolorized with activated charcoal, filtered again through Whatman No. 42 paper, concentrated to $100 \mathrm{ml}$., and allowed to recrystallize in the refrigerator. The product was filtered and dried in vacuo at $75^{\circ} \mathrm{C}$. The water content by Karl Fischer analysis was $0.06 \%$

Tetraethylammonium acetate was prepared by neutralization of tetraethylammonium hydroxide with acetic acid as suggested by Geske (30). The tetraethylammonium hydroxide for this purpose was prepared from tetraethylammonium bromide (Eastman Kodak) and excess silver oxide. It was not possible to remove all the water from the tetraethylammonium acetate even after a thirty-hour evacuation under high vacuum in a freeze dry unit (National Research Corp.). The product was liquid and contained $107 \mathrm{mg}$. of water per $\mathrm{ml}$. This water was removed with acetic anhydride.

The reagent-grade methanol used in these experiments displayed a slight anodic wave at +1.78 volts vs. the s.c.e. in acetonitrile. Other chemicals used in this work were reagent grade, and voltammetrically pure. 
PHENOIS AND OTHER ELECTROACTIVE COMPOUNDS

Isoeugenol (Schimmel and Company) was purified by three distillations under vacuum. The third distillation was conducted under an atmosphere of nitrogen and yielded a colorless product (boiling point: $100^{\circ} \mathrm{C}$. at $0.9 \mathrm{~mm}$. $\mathrm{Hg}$; refractive index: $\left.\underline{n}_{-}^{20}=1.5762\right)$. . Literature values for cis- and trans-isoeugenol are $\underline{\mathrm{n}}_{\underline{\mathrm{D}}}^{20}=1.5726$ and 1.5782 , respectively (45). The purified product was stored in a freezer at about $-20^{\circ} \mathrm{C}$; in a dark glass bottle, but developed a faint yellow color by the time it was used.

The following compounds were recrystallized from water or ethanol-water, dried, and stored over phosphorus pentoxide for voltammetric experiments (the indicated melting points were obtained after recrystallization): 2,4,6-tri-tertbutylphenol (K. and $\mathrm{K}$. Laboratories, m.p. 128.2 - 129.2 ${ }^{\circ} \mathrm{C}$. ); 2,6-di-tert-butylp-cresol (Eastman Kodak, yellow label, m.p. 68.5 - 70.1 ${ }^{\circ} \mathrm{C}$ ); 2,6-di-methoxyphenol (Eastman Kodak, white label, m.p. 53.8 - 56. $0^{\circ} \mathrm{C}$ )); hydroquinone (Matheson, Coleman, and Bell); 2,4,6-trinitrophenol (Baker, U.S.P., m.p. 120 - $122^{\circ}$ C.). For the electrolysis of 2,6-di-tert-butyl-p-cresol the unpurified product (m.p. 68.2.$70.2^{\circ} \mathrm{C}$. ) was used.

The following compounds were used as received: vanillin (Eastman Kodak, white label, m.p. $79.5-82^{\circ} \mathrm{C}$, or Monsanto, U.S.P., m.p. $\left.78-81^{\circ} \mathrm{C}.\right)$; 2,6-ditert-butylphenol (Eastman Kodak, yellow label); dehydrodivanillin (supplied by E. E. Dickey, analytical sample No. 145700). These compounds were voltametrically pure.

The method of cook (46) was used to prepare 3,5,3',5'-tetra-tert-butylistilbene4,4'-quinone (III) by the alkaline ferricyanide oxidation of 2,6-di-tert-butyl-pcresol. After two recrystallizations, the product melted at $305-307^{\circ} \mathrm{C}$. (lit. value 
314-315 C.). A trace contamination, probably from 1,2-bis(3,5-di-tert-butyl-4hydroxyphenyl)-ethane was indicated in the voltammogram of this compound.

The stilbenequinone (III) was reduced with hydrogen on a palladium catalyst to give 1,2-bis (3,5-di-tert-butyl-4-hydroxyphenyl)-ethane (I) (m.p. 168.5-169.5 ${ }^{\circ} \mathrm{C}$. ; lit. value $169-170^{\circ} \mathrm{C}$.) as suggested by Moore and Waters (47). A reduction of the stilbenequinone (III) with zinc in glacial acetic acid as suggested by Cook. (46) produced 3,5,3',5'-tetra-tert-butyl-4, 4'-dihydroxy-stilbene (m.p. 238.5-240' C.; lit. value $\left.240-241^{\circ} \mathrm{C}.\right)$.

3,5-Di-tert-butyl-4-hydroxybenzaldehyde (V) was prepared by the oxidation of 2,6-di-tert-butyl-p-cresol with bromine in tertiary butyl alcohol by the method of Coppinger and Campbell (스). It had a melting point of $185.2-187.8^{\circ} \mathrm{C}$. (1it. value $\left.189^{\circ} \mathrm{C}.\right)$.

3,5-Di-tert-butyl-4-hydroxybenzyl alcohol (IV) was prepared by reduction of the aldehyde (V) with lithium aluminum hydride by the method of Coppinger and Campbell (48) (m.p. 135.8-137. $5^{\circ} \mathrm{C}$; lit. value $137.7-138.1^{\circ} \mathrm{C}$.).

2,6-Di-tert-butyl-1, 4-benzoquinone (VIII) was prepared by oxidation of the aldehyde (V) with lead dioxide in the manner of Cook, et al. (49), (m.p. $65-66.8^{\circ} \mathrm{C}$. , lit. value $65-66^{\circ} \mathrm{C}$.).

3,5-Di-tert-butyl-4-hydroxybenzoic acid (VI) was prepared by a caustic fusion of 3,5-di-tert-butyl-4-hydroxybenzaldehyde in the manner of Yohe, et al. (으). The purified product melted at $205-207^{\circ} \mathrm{C}$. and remelted at $215-217^{\circ} \mathrm{C}$. (lit. value $217-$ $\left.218^{\circ} \mathrm{C}.\right)$. This product had an infrared spectrum identical with that of an authentic sample kindly provided by Dr. G. R. Yohe*.

*Dr. G. R. Yohe, Illinois State Geological Survey, Urbana, Illinois. 
2,6-Di-tert-butyl-4-methyl-4-methoxy-2,5-cyclohexadienone (VII) was prepared by the bromine oxidation of 2,6-di-tert-butyl-p-cresol in methanol, the method of Coppinger and Campbell (48). It had a melting point of $90-91.5^{\circ} \mathrm{C}$. (Iit. value $94^{\circ} \mathrm{C}$.), and contained no impurity which could be detected by gas chromatography or voltammetry.

\section{ELECTRODE PRETREATMENT FOR VOLTAMMETRY}

Voltammetric results at rotating platinum electrodes can be influenced profoundly by the state of oxidation of the platinum surface. A recent review of the literature on platinum surface oxides has been presented by Young (피), so the present discussion will be brief. Kolthoff and Tanaka (르) demonstrated the formation of oxide films on platinum electrodes during anodic voltammetry, and the cathodic dissolution patterns which are characteristic of these films. Anson and Lingane (53) gave chemical evidence for the formation of both platinous and platinic oxides in the anodic treatment of platinum electrodes. Laitinen and Enke (54) have postulated a mechanism based upon chronopotentiometric data for the formation of such films.

Anson (25) has shown that the method of removal of surface oxides from platinum can result in either a lightly platinized reduced surface, or a smooth reduced surface. His results indicate that the presence of a platinized layer may have as great an influence on the observed results as the presence of an oxide film. He also makes the important point that in some previous studies electrode surfaces which were reported as oxidized were in truth reduced either electrochemically or by some chemical reducing agent which was present in the test solution. The general rule seems to be that electrochemical oxidations proceed less reversibly at an oxidized platinum surface than at a reduced surface, and that reactions at a platinized surface are more reversible than at a nonplatinized surface. In some 
reported instances an electrode reaction is completely inhibited, at least before the decomposition of the supporting electrolyte solution by the presence of an oxide film. Lingane ( 26$)$ observed this behavior with oxalic acid in a chronopotentiometric study.

While there is no direct evidence for the formation of oxide films in acetonitrile solutions, Geske (28) has observed cathodic dissolution patterns after anodic treatment of a platinum electrode which he attributed to the formation of an oxide film formed because of the traces of water left in the acetonitrile. The area of the dissolution pattern was increased as larger amounts of water were added to the system.

In the present study, experiments have been conducted on both "oxidized" and "reduced" electrodes. The "oxidized" electrode was achieved by a half-minute treatment in hot chromic acid. This treatment has been found to remove polymeric films formed on the electrode surface in agreement with the results of previous workers $(\underline{8}, \underline{9})$.

A "reduced" electrode was achieved by cathodically pretreating the electrode at a potential of -0.5 volt vs. the s.c.e. for five minutes or until the residual current at this potential dropped to zero. This potential is more negative than the potential at which Geske (28) observed cathodic dissolution patterns for the rotating platinum electrode in acetonitrile. In some cases where a polymer film was indicated on the electrode surface the cathodic pretreatment. was preceded by a half-minute treatment in hot chromic acid, but in the cases of 2,6-di-tert-butyl$\underline{p}$-cresol and its derivatives no such cleaning treatment was needed and the whole series of experiments was conducted with only the cathodic pretreatment. The reduced electrode gave excellent reproducibility for voltammograms rerun in the same test solution. 
It was noted that with the oxidized electrodes a small cathodic current flowed at 0 volt vs. the s.c.e. This current which rapidly decayed to zero as the voltammogram was run was probably caused by partial reduction of the oxidized surface. Total reduction of the surface from this current seems unlikely in view of the dramatic differences observed between an oxidized and a cathodically reduced electrode, and because this potential is more positive than the potential at which Geske observed a cathodic dissolution pattern for a platinum oxide. The small cathodic current at zero volt vs. the s.c.e. was completely absent when a cathodically reduced electrode was used. It was present, however, with an electrode which had not been cathodically pretreated between successive voltammograms. With an electrode on which the oxide film was removed with acidic ferrous ammonium sulfate as recommended by Kolthoff and Tanaka. (르) the small cathodic current at zero volt vs the s.c.e. was greatly reduced or completely absent. However, results on such an electrode were different from those on a cathodically reduced electrode, and in general the cathodic pretreatment seems superior for the compounds of interest in the present investigation. In view of the results of Anson (55) it seems likely that the cathodic pretreatment resulted in a "platinized," reduced surface.

With the carbon electrode, fresh paste was usually packed into the tip before each trial. No special surface pretreatment was necessary.

\section{VOLTAMMETRIC PROCEDURE}

Samples were weighed in a dry box maintained below 20\% (usually 7 to 10\%) relative humidity. The weighed sample of phenol was dissolved and diluted with the supporting electrolyte solution to $10 \mathrm{ml}$. in a volumetric flask. The resulting stock solution was subsequently diluted with the supporting electrolyte solution to prepare the test sample. 
Two methods of dilution were used. The first was conventional in a volumetric flask. The second, and more convenient when several concentrations were to be successively studied, was to add twenty-five milliliters of supporting electrolyte to the voltammetric cell and subsequently add the phenol stock solution, working to successively higher concentrations. Since the stock solutions were relatively concentrated (:.025 to .25M), micropipets could be used for additions and the volume increase of the test solution was relatively small. Because the micropipets were calibrated "to contain," they were rinsed well with test solution after addition of the sample to the cell.

Test solutions were deaerated for at least fifteen minutes before running a voltammogram. The span voltage and cell resistance were measured frequently, the usual procedure being to measure the span voltage before each experiment, and cell resistance after each experiment. Residual current curves were run frequently to check the purity of the system.

For the experiments in 50\% aqueous isopropanol, buffer stock solutions were prepared in water with an ionic strength of one molar. Five milliliters of the buffer, $20 \mathrm{ml}$. of distilled water, and the phenol dissolved in isopropanol were added to a 50-ml. volumetric flask. By diluting to the mark with isopropanol a solution of $0.1 \mathrm{M}$ ionic strength was obtained. The buffers used and the measured apparent $\mathrm{pH}$ after dilution is given in Table II. In order to achieve the proper ionic strength a neutral salt, potassium chloride, was included in some of the solutions along with the main buffer components.

In running voltammograms the direction of polarization was - to + , and the rate of polarization was $2.94 \mathrm{mv}$./sec. except where otherwise noted. 
TABLE II

BUFFER SOLUTIONS

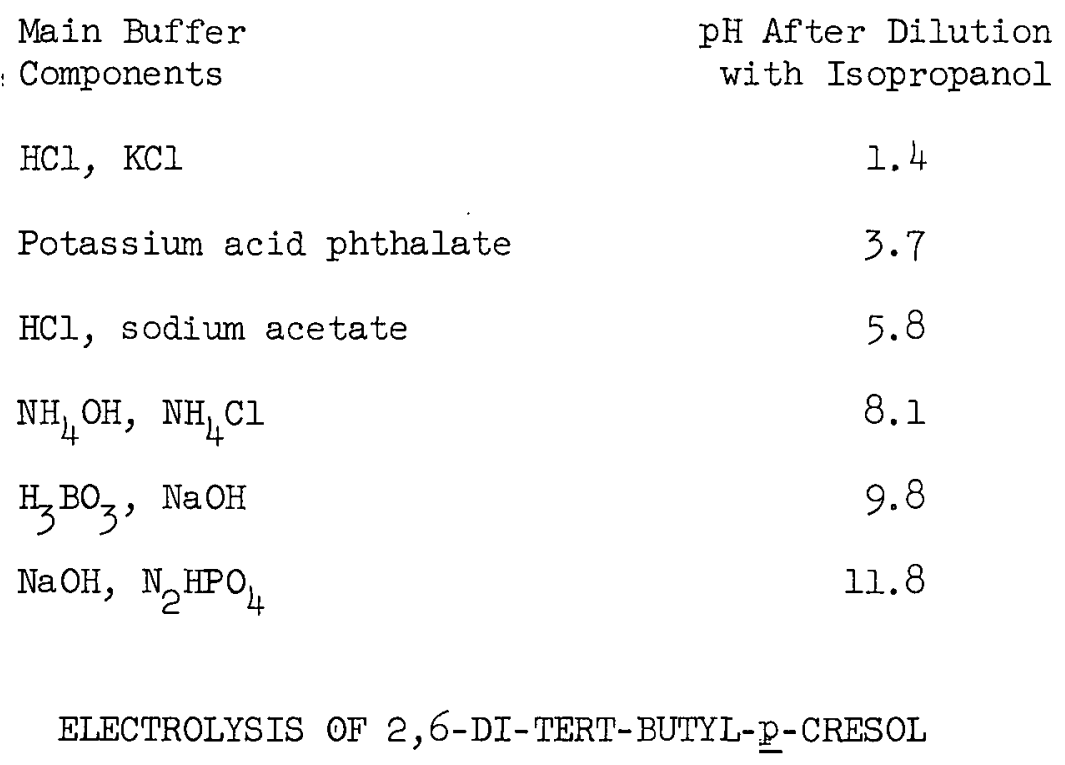

The 2,6-di-tert-butyl-p-cresol (1.100 g.) was dissolved in about $600 \mathrm{ml}$. of $0.5 \mathrm{M}$ sodium perchlorate in the anode compartment of the cell. Current was held constant at $10 \mathrm{ma}$, and methanolic tetraethylammonium hydroxide $(0.6096 \mathrm{~N})$ was added to the anode compartment at a rate of $0.0102 \mathrm{ml}$. per minute, one equivalent of base per Faraday of electricity. A small stream of nitrogen was bubbled continuously through the cell. The anode potential was checked periodically with the student potentiometer.

- The electrolysis was stopped after forty-six hours at which time the electrode potential had risen to 1.55 volts. A $25-\mathrm{ml}$. aliquot of the anolyte was titrated with perchloric acid in-acetic acid-acetonitrile solution (10 ml. of the $0.519 M$ solution diluted to $100 \mathrm{ml}$. with acetonitrile) to the methyl violet end point. From this titration it was determined that $3 \%$ excess base had been added during the electrolysis.

After neutralizing the excess base with perchloric acid in acetic acid, the anolyte was concentrated to dryness using a rotating evaporator with the temperature 
maintained below $28^{\circ} \mathrm{C}$. The residue was extracted thoroughly with ether; the ether fraction was washed with water, dried with anhydrous magnesium sulfate, and concentrated to dryness to yield $1.082 \mathrm{~g}$. of a semicrystalline crude product. Considering that $4.6 \%$ of the original anolyte had been used for preliminary experiments, this represented a yield of $103 \%$ based on the starting $2,6-d i-t-b u t y l-p-c r e s o l$.

A small sample of the crude product was sublimed at reduced pressure (aspirator, $\left.50-60^{\circ} \mathrm{C}.\right)$, and the sublimate was recrystallized from methanol-water to give pale yellow needles, m.p. $89-90^{\circ} \mathrm{C}$. This product had the split carbonyl doublet in the infrared spectrum at $6 \mu$, and the ultraviolet maximum at $234 \mathrm{~m} \mu\left(\epsilon_{\max }=\right.$ 11,200) which is characteristic of the cyclohexadienone configuration (27). The molecular weight was determined by the Rast method to be $265 \pm 5 \%$ (three determinations). An elementary analysis gave C: $77.2 \% ; \mathrm{H}: 10.9 \%$. This product was shown to be identical with synthesized 2,6-di-tert-butyl-4-methyl-4-methoxy-2,5cyclohexadienone by mixed melting point and infrared spectra.

The following analysis for the crude product of oxidation was obtained by gas chromatography on a Carbowax column at temperatures of 188 and $151^{\circ} \mathrm{C}$.

1. 2,6-Di-t-butyl-4-methyl-4-methoxycyclohexadienone: $65.3 \%$.

2. Minor single constituent (single peak by gas chromatography): $10.4 \%$.

3. Five minor constituents (observable by gas chromatography): $8.0 \%$.

4. Nonvolatile (not observable by gas chromatography): $16.3 \%$.

The $10.4 \%$ constituent gave a gas chromatographic peak on the Carbowax column at $150^{\circ} \mathrm{C}$, which was well separated from the other components of the mixture, and therefore was suited to a chromatographic separation. Before attempting the separation the gas chromatograph was set at $205^{\circ} \mathrm{C}$. overnight with a small flow of helium gas to purge impurities from the system, after which the temperature was 
reduced to $150^{\circ} \mathrm{C}$. Ten milligrams of the crude product was dissolved in $100 \lambda$ of reagent-grade benzene and 20 to $40 \lambda$ portions of this solution were injected into the chromatograph. The sample collector was connected to the chromatograph when the peak for the desired component appeared on the recorder chart. The tails at the beginning and the end of the peak were not collected in the interest of purity. Between injections the column was backflushed with helium for a time greater than the elution time of the collected component. The collected sample condensed immediately in the entry tube to the collection device. The product, bright yellow needles, was rinsed into an agate mortar and pestle with a small quantity of dry ether, and after evaporation of the ether a potassium bromide pellet was prepared for an infrared spectrum. The resulting spectrum was identical with that of 2,6-di-tert-butyl-1, 4-benzoquinone.

No further products were isolated from the crude mixture. However, it was noted that the nonsublimable portion of the product contained a carbonyl band in the infrared spectrum at $5.78 \mu$. This was shifted just enough from the carbonyl band of 3,5-di-tert-butyl-4-hydroxybenzoic acid at $5.83 \mu$ to represent an ester of that acid $(\underline{58})$.

\section{ELECTROLYSIS OF THE VANILLINATE ANION}

An initial electrolysis of the vanillinate anion was conducted in the millicoulometric cell on a l-ml. sample which was $1.32 \mathrm{mM}$ in vanillin and in aqueous tetraethylammonium hydroxide. The supporting electrolyte-solvent system was $0.5 \mathrm{M}$ tetraethylammonium perchlorate in acetonitrile. The electrolysis was conducted at +0.39 volt vs. s.c.e. for 2.5 hours with a consumption of 0.0964 coulomb of electricity. At the end of the electrolysis the anolyte was removed from the cell and acidified with glacial acetic acid. After standing for several days some fine white needle-shaped crystals separated from the solution. 
A second electrolysis was conducted in the millicoulometric cell on a l-ml. sample which was $50.4 \mathrm{mM}$ in vanillin and tetraethylammonium hydroxide, again with $0.5 \mathrm{M}$ tetraethylammonium perchlorate as a supporting electrolyte in acetonitrile. At the end of 4.38 hours the current level had fallen to one-tenth of its initial value and the electrolysis was stopped. The anolyte was removed from the cell and acidified with glacial acetic acid. The crystals from the first electrolysis experiment were combined with this to hasten crystal growth. After cooling in a refrigerator, the precipitated product was centrifuged in a $3-\mathrm{ml}$. test tube and the yellow supernatant liquid was decanted. The initial precipitate was washed with two $0.5-\mathrm{ml}$. portions of acetonitrile, redissolved in acetonitrile by..addition of a few drops of tetraethylammonium hydroxide, reprecipitated with glacial acetic acid, filtered, washed with distilled water, and dried. The product had an infrared spectrum identical with that of dehydrodivanillin, and melted at $308-310^{\circ} \mathrm{C}$, decomp. [reported value for authentic specimen $313-317^{\circ} \mathrm{C}$. (59)].

In a large-scale electrolysis $0.7617 \mathrm{~g}$. of vanillin was dissolved in $500 \mathrm{ml}$. of an $0.5 \mathrm{M}$ solution of sodium perchlorate in acetonitrile. After this solution was added to the anode compartment, enough acetonitrile was added to bring the anolyte level above the frit leading to the cathode compartment. For this electrolysis the rotating platinum electrode from the voltammetric circuit was set directly into the anode compartment. so that voltammograms could be run as the electrolysis proceeded. The rotating platinum electrode was cleaned in hot chromic acid, and then cathodically reduced for each curve. An initial voltammogram showed that the solution was voltammetrically pure in the region where the vanillinate anion would react. Aqueous tetraethylammonium hydroxide ( $7: 5 \mathrm{ml}$. of ; $0.673 \underline{N}$ ) was added to the anode compartment in a quantity equivalent to the vanillin present. 
The control potential during the electrolysis was to. 42 volt vs. the s.c.e. At intervals during the electrolysis additional tetraethylammonium hydroxide (TEAH) was added in a quantity which was equivalent to one-half the coulombs of electricity consumed since the last addition. The amounts of base added are given in Table III. A small stream of nitrogen was bubbled into the anode compartment throughout the electrolysis.

TABLE III

\section{ELECTROLYSIS OF VANILLINATE ANION}

\begin{tabular}{|c|c|c|}
\hline $\begin{array}{l}\text { Electrolysis } \\
\text { Time, min. }\end{array}$ & $\begin{array}{c}\text { Electricity Consumed, } \\
\text { coulombs }\end{array}$ & $\begin{array}{l}\text { Base Added, } \\
\mathrm{ml} \text {. of } 0.673 \mathrm{TEAH}\end{array}$ \\
\hline 178 & 152.3 & 1.1 \\
\hline 364 & 289 & 1.0 \\
\hline 523 & 402 & 0.8 \\
\hline 639 & 551 & -- \\
\hline & & $2.9 \mathrm{ml}$. \\
\hline
\end{tabular}

The anolyte was acidified with $5 \mathrm{ml}$. of glacial acetic acid, and, after standing in a refrigerator for four hours, some product precipitated. Upon acidification with a few drops of perchloric acid and further cooling overnight in a refrigerator more product was recovered. It was filtered and air dried (total crude yield 0.4981 g., 65.4\%). About half of this crude product was slurried in $400 \mathrm{ml}$. of ethanol and water, and tetraethylammonium hydroxide was added to effect solution. After filtering off a small amount of insoluble matter, the product was reprecipitated with $0.05 \mathrm{~N} \mathrm{HCl}$. It was shown to be dehydrodivanillin by its infrared spectrum.

The remaining anolyte was concentrated to dryness at $20-30^{\circ} \mathrm{C}$. on a rotating evaporator. Then $100 \mathrm{ml}$. of water was added to dissolve the supporting electrolyte. 
This solution was extracted thoroughly with ether. A small part of the product ( $0.07 \mathrm{g.}, 9.2 \%$, insoluble in either ether or water, and light brown in color) was discarded. The ether solution was dried with anhydrous magnesium sulfate. Paper chromatograms of the ether extract revealed a relatively heavy vanillin spot and no other identifiable product. There was some material present, however, which showed as a pink fluorescent spot $\left(\underline{R}_{\underline{f}}=0.71\right.$ in butanol, pyridine, water, 10:3:3; $\underline{R}_{\underline{f}}=0.72$ in butanol saturated with $2 \%$ aqueous ammonia). This substance showed no color reaction with either diazotized para-nitroaniline or with 2,4-dinitrophenylhydrazine hydrochloride. When the ether extract was concentrated to dryness a black tar resulted. 
VOLTAMMETRY: THEORY, AND ANALYSIS OF DATA

The basic definitions, and the equations for voltammetrically reversible and irreversible waves are included in this section, as well as an explanation of the method of data analysis which was used in the present investigation. Before proceeding further a note on sign conventions is in order. Potentials correspond to the so-called European convention for reporting oxidation reduction potentials (60): Cathodic currents are positive, and anodic currents are negative in accord with normal voltammetric practice. Following the suggestion of Larson, Iwamoto,

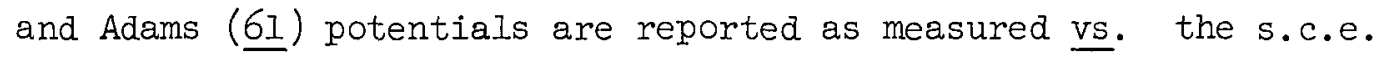

The equations given in this section are based on the concept of a distinct diffusion layer around the rotating electrode and linear concentration gradients within this layer. Such a treatment is notably lacking in rigor, but in practice It is found that the equations for potential, (4) and (6), conform to the theory, and that the diffusion current is proportional to the concentration and the number of electrons involved in the electrode reaction as predicted by Equation (2). The diffusion layer thickness, $\delta$, however, is in reality a function of diffusivity as well as hydrodynamic variables $(\underline{62}, \underline{63})$ so that the diffusion current is proportional to the 0.66 power rather than to the first power of diffusivity as would be predicted by Equation (2). Furthermore, unless a perfectly cylinarical electrode were used, it is not to be expected that current would be truly proportional to the electrode surface area.

A general equation for the electro-oxidation of an organic compound with hydrogen ion evolved in the electrode process may be written

$$
\mathrm{R} \underset{\mathrm{k}, \mathrm{k}, \mathrm{b}}{\stackrel{\mathrm{k}, \mathrm{h}}{\rightleftarrows}} \mathrm{O}+\mathrm{ne}^{-}+\mathrm{H}^{+}
$$


where $\underline{R}$ represents the reduced and $\underline{0}$ the oxidized species, $\underline{\mathrm{n}}$ is the number of electrons involved in the reaction, and $\underline{k_{\underline{b}}, \underline{h}}$ and $\underline{\underline{k}}_{\underline{f}, \underline{h}}$ represent the heterogeneous rate constants for the oxidation and reduction, respectively. These are formal rate constants; i.e., they are to be used in kinetic equations with concentrations rather than activities. The terminology is according to Delahay (느).

When the heterogeneous rate constants are sufficiently large, the observed results are close to the predicted results assuming that an equilibrium exists between the oxidized and reduced species at the electrode surface at all times. Under those conditions the Nernst equation can be applied and the system is said to be "voltammetrically reversible." This does not imply reversibility in the thermodynamic sense. In this case the following approximate equations, developed in the manner described by Delahay (느), for stirred solutions apply:

$$
\begin{gathered}
i_{D}=-n F A D_{R} C_{R}^{O} / \delta_{R} \\
E_{l / 2}=E_{0}-\frac{R T}{n F} \ln \frac{D_{0} \delta_{R} f_{R} D_{H^{+}}}{D_{R} \delta_{O} f_{0} \delta_{H^{+}}}-\frac{R T}{n F} p H \\
E=E_{l / 2}+\frac{R T}{n F} \ln \left[i /\left(i_{D}-i\right)\right]
\end{gathered}
$$

where

$$
\begin{aligned}
& \underline{\underline{i}}_{\underline{D}}=\text { diffusion current } \\
& \underline{\underline{B}}=\text { number of electrons involved in the electrode process } \\
& \underline{F} \quad=\text { Faraday constant } \\
& \underline{A} \quad=\text { electrode surface area } \\
& \underline{\mathrm{D}}_{\mathrm{R}}=\text { diffusivity of the reduced species } \\
& \underline{\mathrm{C}}_{\underline{\mathrm{R}}}^{\mathrm{O}}=\text { concentration of reduced species in the bulk of the solution } \\
& \underline{\delta}_{\underline{R}}=\text { diffusion layer thickness, reduced species }
\end{aligned}
$$




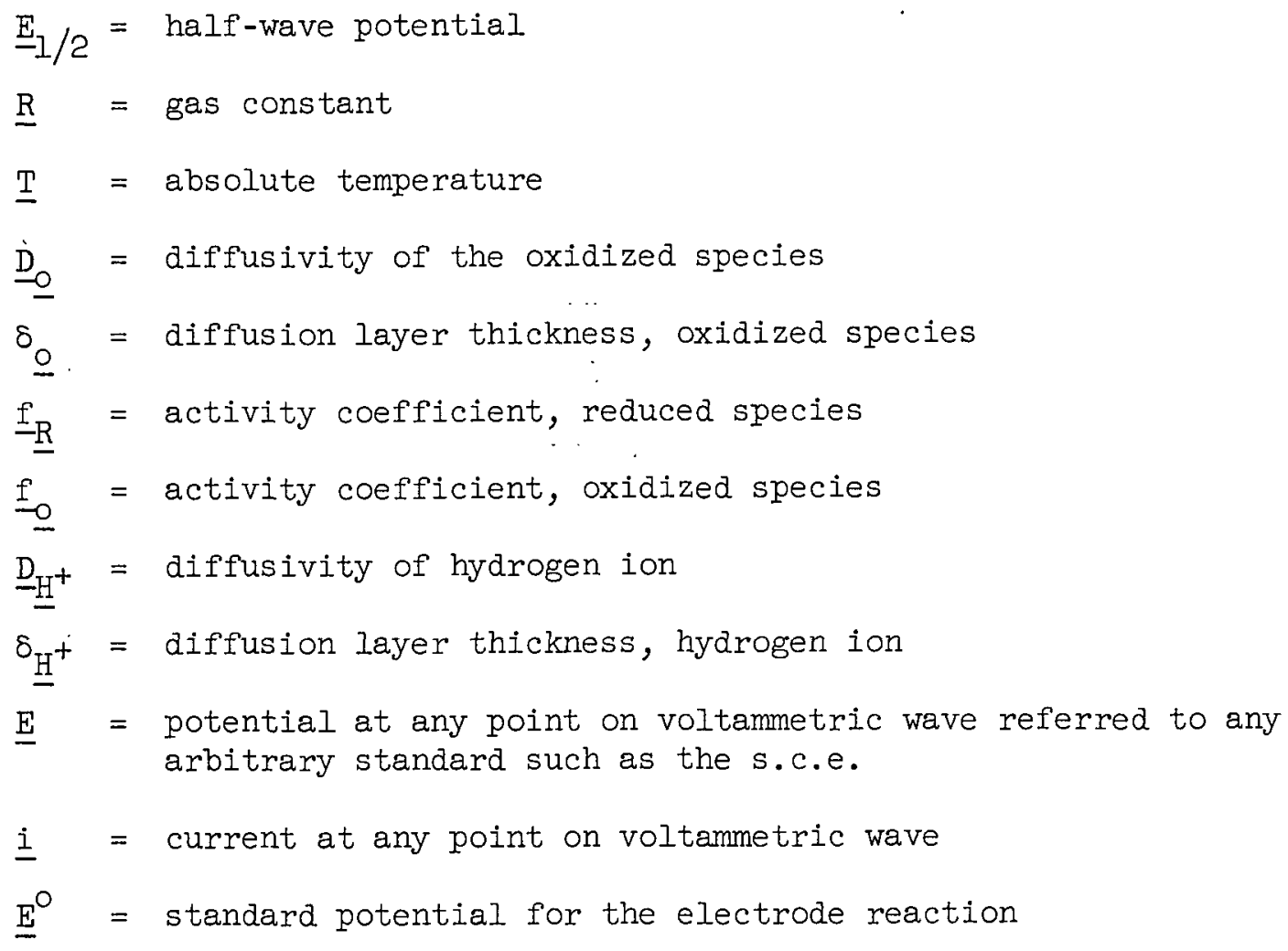

When the rate of the reverse reaction is sufficiently slow so that it can be neglected, the electrode reaction is said to be "totally irreversible." Equation (2) for the current is still applicable, but Equations (3) and (4) are replaced by (5) and (6) as follows:

$$
\begin{aligned}
E_{I / 2}= & E_{c}^{O}-\frac{R T}{\beta n_{\beta} F} \ln \left(k_{s, h} \delta_{R} / D_{R}\right) \\
E & =E_{I / 2}+\frac{R T}{\beta n_{\beta} F} \ln \left[i /\left(i_{D}-i\right)\right]
\end{aligned}
$$

where

$$
\begin{aligned}
& \underline{E}_{c}^{\circ}=\begin{array}{l}
\text { potential at which chemical equilibrium exists at the electrode } \\
\text { surface }
\end{array} \\
& \beta=\text { transfer coefficient* }
\end{aligned}
$$

*The symbol $\beta$ has been used for the oxidation reaction to distinguish this from the transfer coefficient for the reduction reaction $(1-\alpha)$; i.e., $\beta$ is not necessarily the same as $(1-\alpha)$ (42). 
$\underline{n}_{-\beta}=$ electrons transferred in the rate-controlling step

$\underline{\mathrm{k}}_{\underline{\mathrm{s}}, \underline{\mathrm{h}}}=\underline{\underline{k}}_{\underline{\mathrm{b}}, \underline{\mathrm{h}}}^{\mathrm{o}} \exp \left[\begin{array}{ll}\frac{\beta \underline{\underline{n}}}{-\beta} \underline{F} & \underline{\mathrm{R}} \\ \underline{\underline{T}}\end{array}\right]$

$\underline{\mathrm{k}_{\mathrm{b}} \mathrm{O}} \underline{\mathrm{h}}=$. rate constant for the oxidation reaction when $\underline{E}=0$.

An interesting point to note is that the half-wave potential for a totally irreversible electrode reaction [Equation (5)] is not a function of $\mathrm{pH}$ even though hydrogen ions are evolved in the electrode process.

According to Delahay ( $\underline{42}$ ) the range of values for $\underline{k}_{\underline{s}}, \underline{h}$ where the electrode reaction cannot be treated as either reversible or totally irreversible is relatively narrow. This is fortunate because in the intermediate range between the extremes of reversible and irreversible behavior the theory is considerably more complex.

When the voltammetric wave is "normally" shaped the treatment of data is straightforward. After correction for the residual current and $\underline{\text { iR }}$ drop the wave will look something like Curve 1 of Fig. 7 for the reversible case, or Curve 2 for the irreversible case. In either of these circumstances the diffusion current is readily chosen as the height of the diffusion plateau, and the half-wave potential is the potential at which the current level is $\underline{i} \underline{D} / 2$.

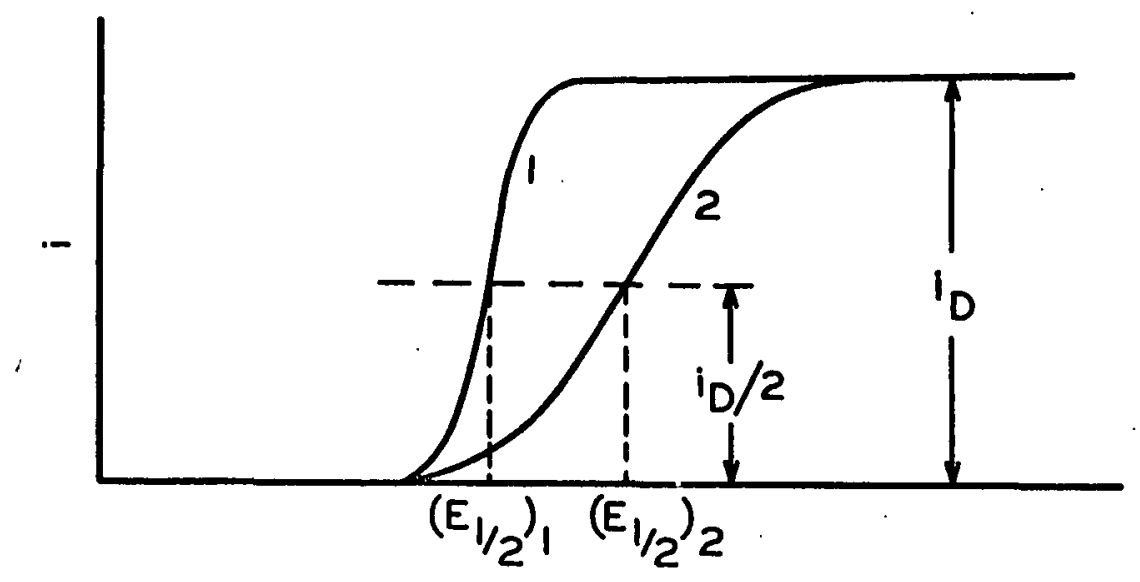

Figure 7. Normally Shaped Voltammograms 
With many organic compounds, however, interpretation is much more difficult because, as shown in Fig. 8, the current reaches no distinct plateau but instead continues to rise. In this case the selection of a diffusion current is an arbitrary matter, and therefore both $\underline{\underline{i}}_{\underline{D}}$ and $\underline{E}_{1 / 2}$ depend upon the whims of the investigator.

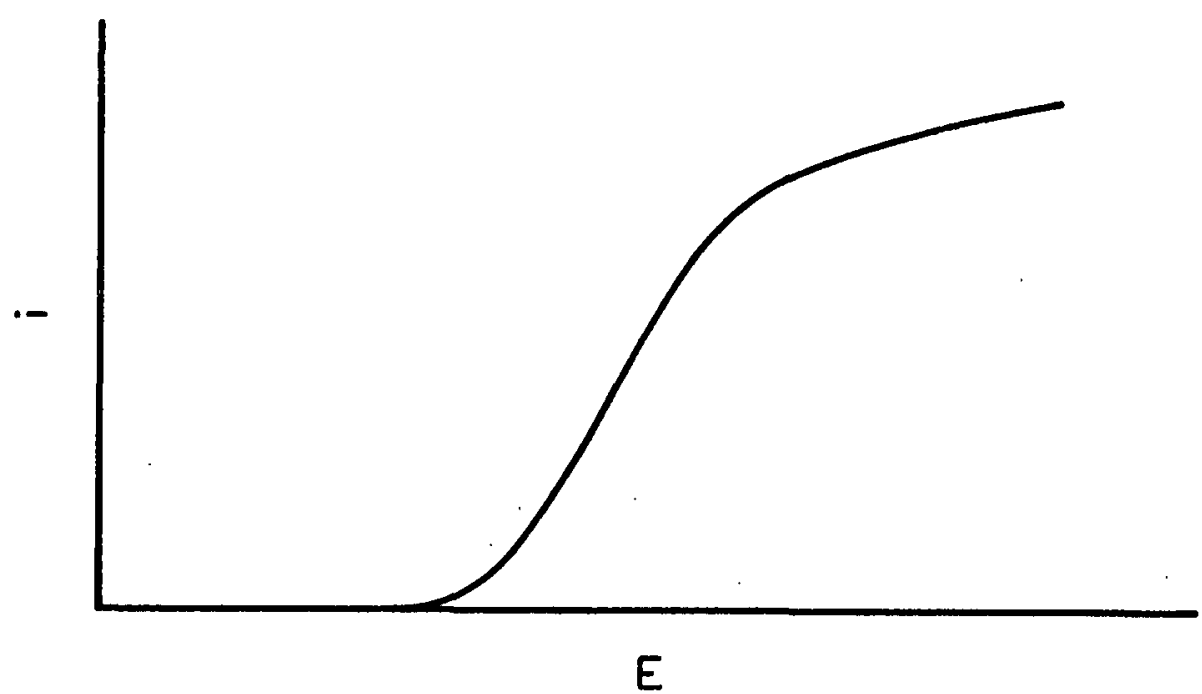

Figure 8. Abnormally Shaped Voltammogram

A possibility for the uniform treatment of such curves comes from the fact that for either the "reversible" case, Equation (4), or the "totally irreversible" case, Equation (6), a plot of the function $\log \underline{\underline{i}} /\left(\underline{i}_{\underline{D}}-\underline{i}\right)$ vs. E should yield a straight line. By the use of a trial and error calculation $\underline{i}_{\underline{D}}$ may be chosen as the current which gives the best least squares fit to a straight line in a plot of $\underline{i} /\left(\underline{i}_{\underline{D}}-\underline{i}\right)$ vs. $\underline{E}_{0}$ This is the basis used in the development of a computer program (IBM 1620) to process voltammetric data. The program was useful even in the case of the "normally" shaped waves because it corrects the data curves for residual current and $\underline{i} \underline{R}$ drop and provides values of $\underline{\mathrm{n}}$ or $\beta_{-\beta}$, respectively, in Equations (4) and (6).*

*In tabulations of data given in later sections of this thesis the slope of the plot of $i /\left(\underline{i}_{-}-\underline{i}\right)$ vs. E after multiplication by 0.0591 is referred to as $\beta \underline{n}_{\beta}$ regardless of whether a reversible or an irreversible wave is being considered. 
It is realized by the author that the trial and error basis for selecting $\underline{i}_{\underline{D}}$ is not strictly applicable in the case midway between "reversible" and "totally irreversible" behavior. In this case, as well as in the case of kinetic or catalytic waves, the computed value of $\underline{n}$ has no theoretical significance. However, since the waves of interest in this study did fit an equation of the form of Equation (4) or (6) quite well the calculated value of $\beta_{n_{\beta}}$ has practical use since it at least empirically describes the wave form. 


\section{PHENOXONIUM ION ${ }^{1}$ MECHANISM. IN ACETONITRILE}

Initial voltammetric studies with a number of phenols gave diffusion currents which, by comparison with hydroquinone ${ }^{2}$, indicated approximately a two-electron transfer. Since the phenolic hydroxyl should be practically undissociated in acetonitrile, it was reasonable to presume that the nonionized phenol was reacting, and it was hypothesized that the primary electrode process involved the formation of a mesomeric phenoxonium ion according to Equation (7). The calculated values of $\beta_{-\beta}$ were in the range from 0.48 to 0.72 depending on the condition of the

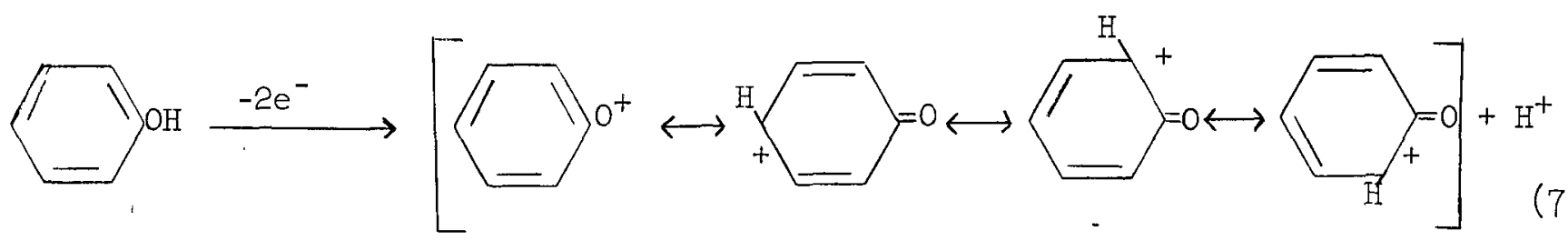

electrode surface and the concentration for both the phenols and hydroquinone. This indicated an irreversible electrode process. The effect of an oxide film on the surface of the platinum electrode was pronounced, an oxidized surface giving a half-wave potential as much as 0.1 volt lower than a reduced surface. There was also a decrease in the apparent reversibility of the reaction on an oxidized surface, but.no significant effect on the diffusion current. With isoeugenol severe filming was encountered as noted in the Appendix, but with the hindered phenols and other phenols which had two or more of the reactive ortho and para positions blocked, no filming was encountered.

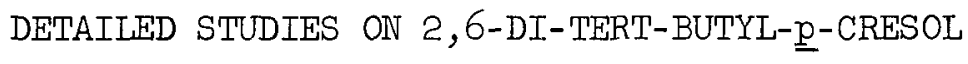

The hindered phenol, 2,6-di-tert-butyl-p-cresol (2,6-DTBC), was selected for intensive study because the reactions of this compound with chemical oxidizing

\footnotetext{
The term, "phenoxonium ion," evidently has not been used previously in the chemical literature. In this thesis it refers to a mesomeric onium ion of the type shown in Equation (7).

2 The results of Lund $(31,32)$ and Geske (29, 30) have indicated indirectly that the anodic reaction of hydroquinone in acetonitrile is a two-electron process.
} 
agents are quite well known. All of the voltammetric studies in this section were conducted on Electrode No. V-3 with a reduced surface. The'supporting electrolyte was in all cases $0.5 \mathrm{M}$ tetraethylammonium perchlorate.

\section{EFFECT OF CONCENTRATION}

Results of a number of voltammetric experiments at different concentrations of 2,6-DTBC are given in Table IV. Three points of interest will be noted in these data. First, the diffusion current constant for the main wave increases with concentration. Second, there is a second wave which is absent at very low levels of concentration, but occurs at intermediate levels of concentration and becomes more pronounced at high levels of concentrations. Third, $E_{1 / 2}$ for the main wave seems to increase and then to decrease as the concentration is increased.

The values of $\underline{i}_{\underline{D}} / \underline{C}$ for the main wave are plotted against concentration in Fig. 9. It will be noted that $\underline{\underline{i}}_{\underline{\mathrm{D}}} / \underline{\mathrm{C}}$ increases numerically with increasing concentration and then levels off at concentrations greater than ImM. It is expected that if the data could be extended to still lower levels of concentration the curve of Fig. 9 would again level off, probably without reaching a significantly lower value. The ratio of ${ }_{{ }^{i}} \underline{D} / \underline{C}$ at the highest level of concentration to $\underline{i}_{\underline{D}} / \underline{C}$ at the lowest level of concentration is 1.48 from the smoothed curve of Fig. 9. This would suggest a three-electron and a two-electron limiting over-all reaction at the extremes of concentration.

The second wave of $2,6-$ DTBC is shown well in Fig. 10 where $\underline{i} / \underline{C}$ is plotted against potential at representative levels of concentration. It will be noted that this second wave does not occur at concentration levels below $0.5 \mathrm{mM}$, and that its height becomes greater with increasing concentrations. The second wave was not always observed in the concentration range from 0.5 to $1.0 \mathrm{mM}$. This was probably caused by impurities in the acetonitrile. On the assumption that the value of 


\section{TABLE IV}

EFFECT OF CONCENTRATION ON THE VOLTAMMETRY

OF 2,6-DI-TERT-BUTYL-p-CRESOL

(Electrode V-3, reduced surface)

Wave I

Conen., $\underline{\mathrm{mM}}$

0.0210

0.0312

0.0416

0.0518

0.0528

0.106

0.211

0.528

0.528

0.901

0.901

1.06

1.58

3.64

6.68

9.66
$E_{I / 2}$ $-\underline{i} \underline{D} / \underline{C}$

$\mu \mathrm{a} . / \mathrm{mM}$ volts vs. s.c.e.

9.5

$9 \cdot 9$

10.6

11.4

10.4

12.1

13.7

13.3

12.6

13.5

13.4

14.6

13.1

14.6

14.5

13.7
1.21

1.21

1.19

1.20

1.23

1.25

1.27

1.25

1.22

1.24

1.27

1.26

1.25

1.24

1.22

1.21
Wave II

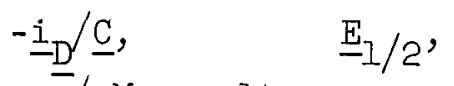

$\mu a_{\circ} / \underline{m}$ volts vs. s.c.e.

$\beta n_{\beta}$

$-$
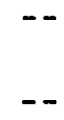

0.701

0.614

0.592

0.620

1.6

1.63

0.505

0.678

2.7

1.58

0.445

$0.556 \quad 2.4$

1.61

0.525

$0.730 \quad 2.5$

1.59

0.404

$0.545 \quad 3.0$

1.63

0.605

0.660

3.7

1.62

0.491

0.705

6.3

1.60

0.431

0.826

8.3

1.53

0.511

0.861

9.4

1. 54

0.467 


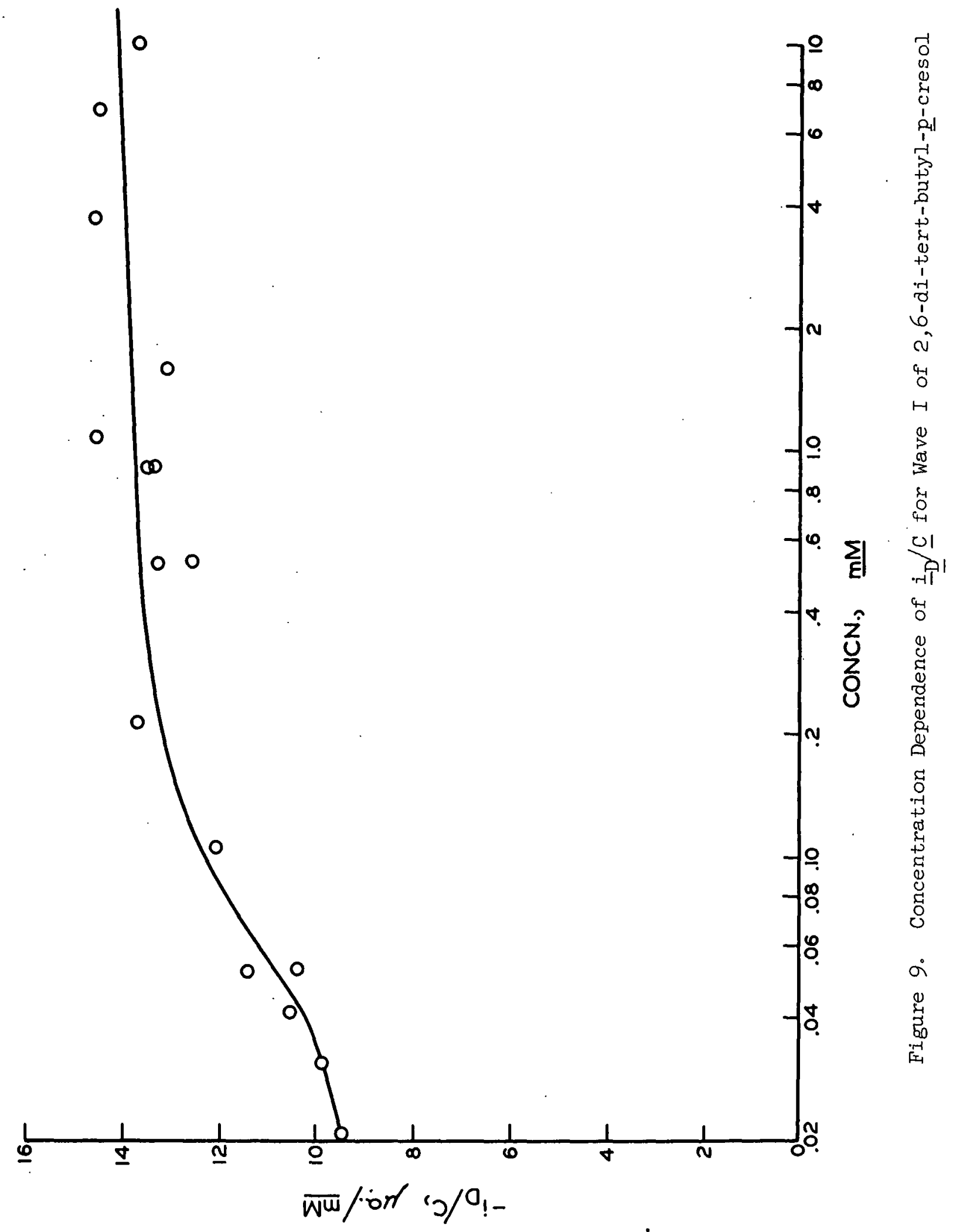




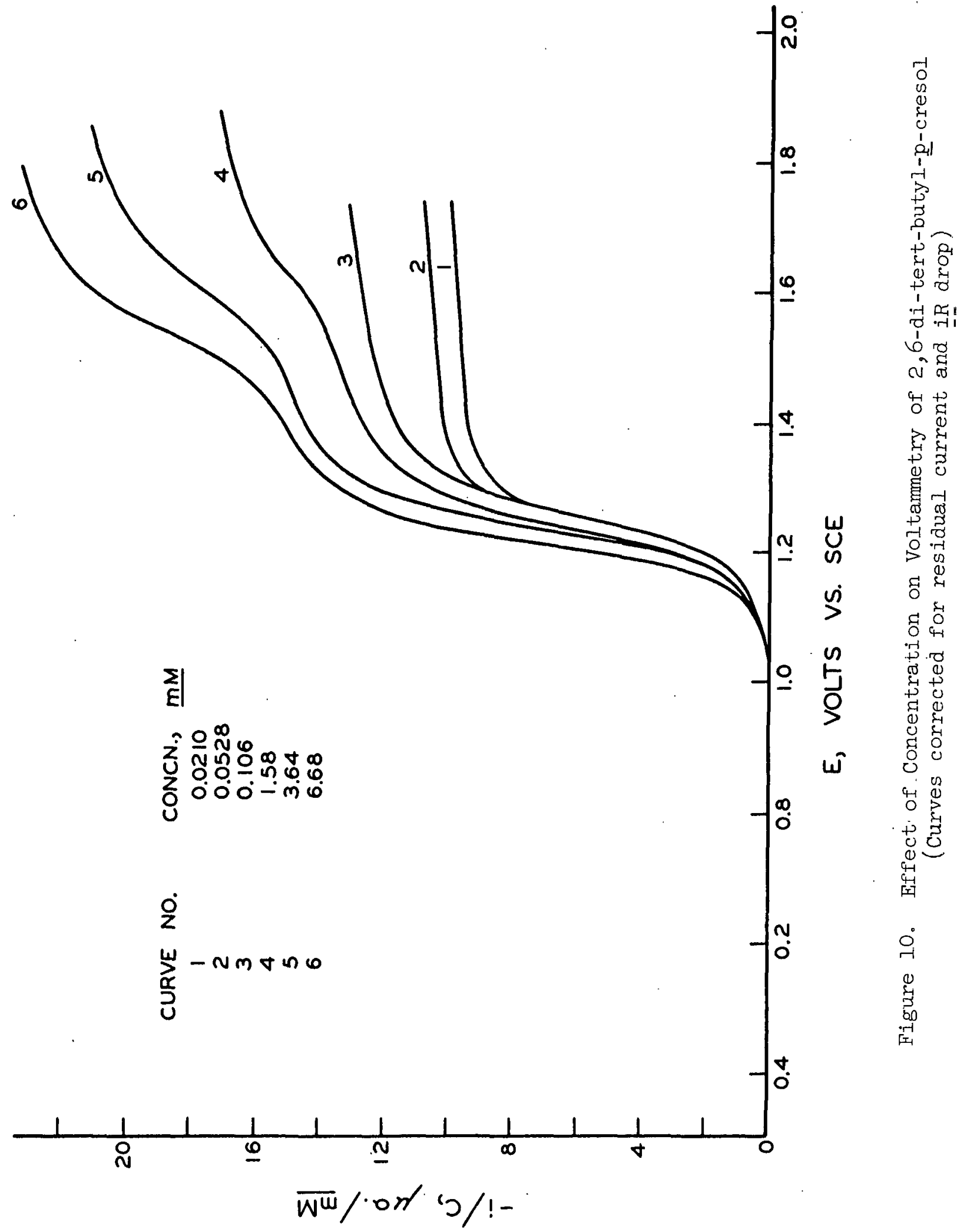


${ }_{\underline{i}} / \underline{C}$ for the first wave represents a three-electron transfer at the higher levels of concentration, this second wave approaches the value for a two-electron transfer at the highest level of concentration.

The increasing value of $-\underline{i} \underline{D}$ 을 for the main wave of 2,6-DTBC and the appearance of the second wave at high levels of concentration, but not at low levels of concentration can be explained by the reoxidation of a product of reaction after some kinetic process which follows the primary electron transfer. Since the second wave should be characteristic of an oxidation product of 2,6-DTBC, known products from chemical oxidations of 2,6-DTBC were investigated with the results given in Table V. These results. indicate that of the compounds investigated only the dimers, I, II, and III, give waves at approximately the same potential as the second wave of 2,6DTBC at the higher levels of concentration. At lower levels of concentration the water content of the acetonitrile was high enough so that the alcohol IV, and aldehyde $V$, might have also been formed to a limited extent and this may account for the shift of the second wave of 2,6-DTBC to a somewhat higher potential at lower concentrations.

The reaction sequence* given by Equations (8)-(12) could account for the formation of the stilbenequinone, III, from the anodic reaction of 2,6-DTBC. In accord with the experimental evidence this reaction scheme would give an $\underline{\mathrm{n}}$ value of two at low levels of concentration where Reaction (ll) would not occur quickly enough for the electrode Reaction (12) to influence the current level.

In further agreement with the observed results the limiting $\underline{\mathrm{n}}$ value at high concentrations where Reactions (11) and (12) would occur rapidly at the electrode

\footnotetext{
*This reaction sequence was chosen because of the work of Fujisaki (64-67) who has given evidence for the methylenequinone, IX, as an intermediate in this type of reaction.
} 


\section{TABLE V}

VOLTAMMETRY OF PRODUCTS FROM CHEMICAL OXIDATIONS OF 2,6-DTBC

(Electrode $\mathrm{V}-3$, reduced surface)

$$
\begin{aligned}
& \text { Compound } \\
& (+=t-B u)
\end{aligned}
$$

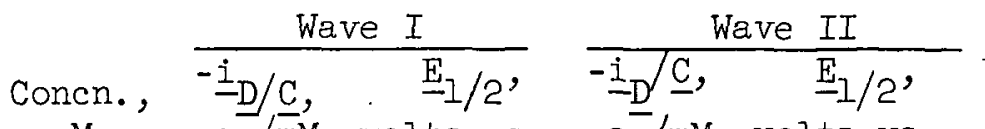

$$
\begin{aligned}
& \underline{\mathrm{mM}} \mu \mathrm{a} . / \overline{\mathrm{mM}} \text { volts vs. } \mu \mathrm{a}^{-} / \underline{\mathrm{mM}} \text { volts vs } \\
& \text { s.c.e. }
\end{aligned}
$$

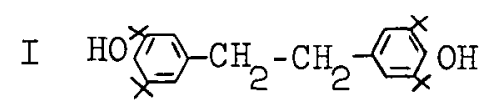

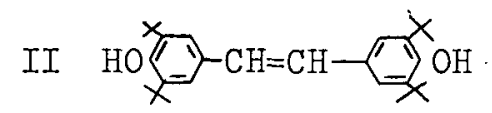

III

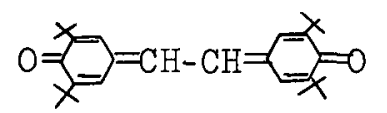

IV

$$
\mathrm{HO} \mathrm{CH}_{2} \mathrm{OH}
$$

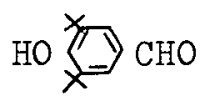

VI $\quad \mathrm{HO} \bigcup_{-} \mathrm{COOH}$

VII<smiles>COc1ccc([18OH])cc1</smiles>

VIII
1.23

0.77

19.7

9.1

0.77

a

$$
--
$$

0.174

1.00

14.3
13.2

1.10

0.586

$-$

-

$-$

No wave observed

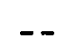

1.25

1.24
No wave observed
1.23
5.8
1.51

9.1

1.53

\section{a}

1.53

3.2

1.72

No wave observed

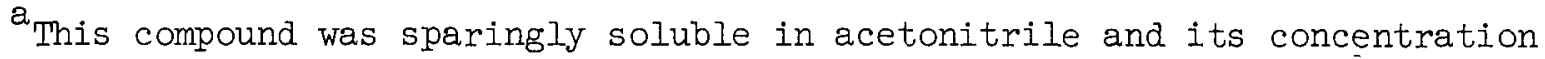
was not known with certainty. However, there is little doubt that this was a two-electron wave; i.e., $\underline{i}_{\underline{D}} / \underline{\mathrm{C}} \sim-9 \mu \mathrm{a} . / \mathrm{mM}$.

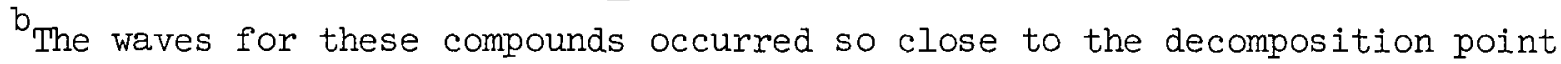
of the solvent supporting electrolyte system that a precise evaluation of $\underline{i}_{\underline{D}} / \underline{C}$ and $\underline{E}_{I / 2}$ was impossible. 
surface would be three electrons per molecule of 2,6-DTBC. For the proposed reaction sequence to be valid, Reaction (11) would have to occur at the same or a lower potential than the main wave for 2,6-DTBC. Results with the dimers, I, and II, indicate that this would be the case.

$$
(+=\mathrm{t} \text {-butyl })
$$

The one point of disagreement with the proposed reaction scheme is that from the results with the dimers, I, II, and III the second wave of 2,6-DTBC should represent a one-electron transfer. However, a two-electron transfer was indicated at the highest level of concentration in Table IV. This may be the result of the excess hydrogen ion generated at the electrode surface which is known to promote the oxidation of the stilbenequinone, III. A discussion of the effect of excess hydrogen ion on the stilbenequinone is given on page 52 .

Altogether there seems to be good evidence for the proposed dimerization scheme. Since no products of this reaction have been isolated and identified, other possibilities cannot be ruled out, although it is difficult to conceive of 
other reaction sequences which will yield a net $\underline{n}$ value of three electrons per molecule in the high range of concentration.

In Table IV, the half-wave potential for the main wave of 2,6-DTBC apparently increases from a value of 1.20 volts at a concentration level of $0.02 \mathrm{mM}$ to a value of 1.27 volts at a concentration level of $0.21 \mathrm{lmM}$. Referring to Fig. 10 (Curves 1, 2, and 3) it will be seen that these waves are really superimposed on one another, and that the increase in $E_{I / 2}$ with concentration is simply an effect of the higher numerical value of $\underline{\underline{i}} \underline{\underline{C}}$.

In the high range of concentration from 0.9 to $9.66 \mathrm{mM}$ in Table IV the halfwave potential apparently decreases from 1.27 to I.2I vol.ts. For a reversible process where hydrogen ion is liberated at the electrode in an unbuffered solution it can be shown that there will be a shift of $\mathrm{E}_{1 / 2}$ to higher values with increasing concentration (37), the opposite of the effect observed here. Since a shift to higher values did not occur, there is evidence that the electrode process is in fact totally irreversible. The observed shift to slightly lower potentials may be explained by the consideration that the rearrangement of the phenoxonium ion to the benzyl carbonium ion in Equations (9) and (10) is promoted by the presence of hydrogen ion. Such an effect has been noted by Cook in the rearrangement of 2,6di-tert-butyl-4-methyl-4-bromo-2,5-cyclohexadienone to 3,5-di-tert-butyl-4-hydroxybenzylbromide (46). Since hydrogen ion is generated in Reactions (8), (11), and (12), it is to be expected that at high levels of concentration there will be an overconcentration of acid in the vicinity of the electrode, and Reactions (9) and (10) will be facilitated. The observed effect on the voltammetric wave of 2,6-DTBC would be to give a higher level of current at the beginning of the wave, but the same limiting current on the diffusion plateau, i.e., an apparent decrease in $E_{1 / 2}$ as observed experimentally. 
EFFECT OF HYDROGEN ION CONCENTRATION

To further investigate the effects of hydrogen ion on the voltammetric behavior of 2,6-DTBC, experiments were conducted in which anhydrous perchloric acid in glacial acetic acid was added to the test solution. Perchloric acid was used because it is the only one of the common strong acids which is completely dissociated in acetonitrile (36). Results of these experiments are shown in Fig. 11 and 12. The main effect on the voltammetric wave with acid concentrations up to $1.36 \mathrm{M}$ is to shift the wave to a less positive potential. This is similar to the effect noted with increasing concentrations of 2,6-DTBC, and is attributed to the same cause, i.e., the effect of acid on the rearrangement of the phenoxonium ion to the benzyl carbonium ion.

When the acid concentration is increased to $11.4 \mathrm{mM}$ in Fig. 11 the wave shape is altered entirely. The same effect is noted at a higher concentration of 2,6DTBC (Fig. 12). This indicates that the reaction path for the oxidation of 2,6DTBC is changed completely in the presence of excess hydrogen ion.

The voltammetric behavior of the dimeric stilbenequinone (III) is dramatically changed in the presence of excess perchloric acid. When the acid is added to the solution a bright red color develops immediately. In this condition new waves are apparent at 0.77 and 1.30 volts. A reasonable explanation for this behavior is that hydrogen ion adds to the stilbenequinone as shown in Equations (13) and (14). The resulting adduct, $\mathrm{X}$, has essentially the same double bonded grouping as the stilbene, II, and this could explain the wave at 0.77 volt. The wave at 1.30 volts could be caused by the new phenolic hydroxyl formed on one end of the molecule in the adduct.

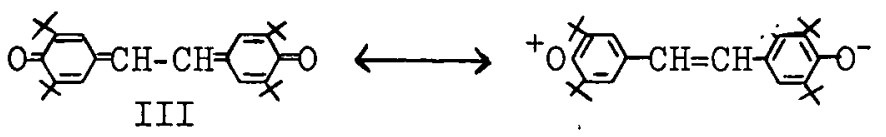




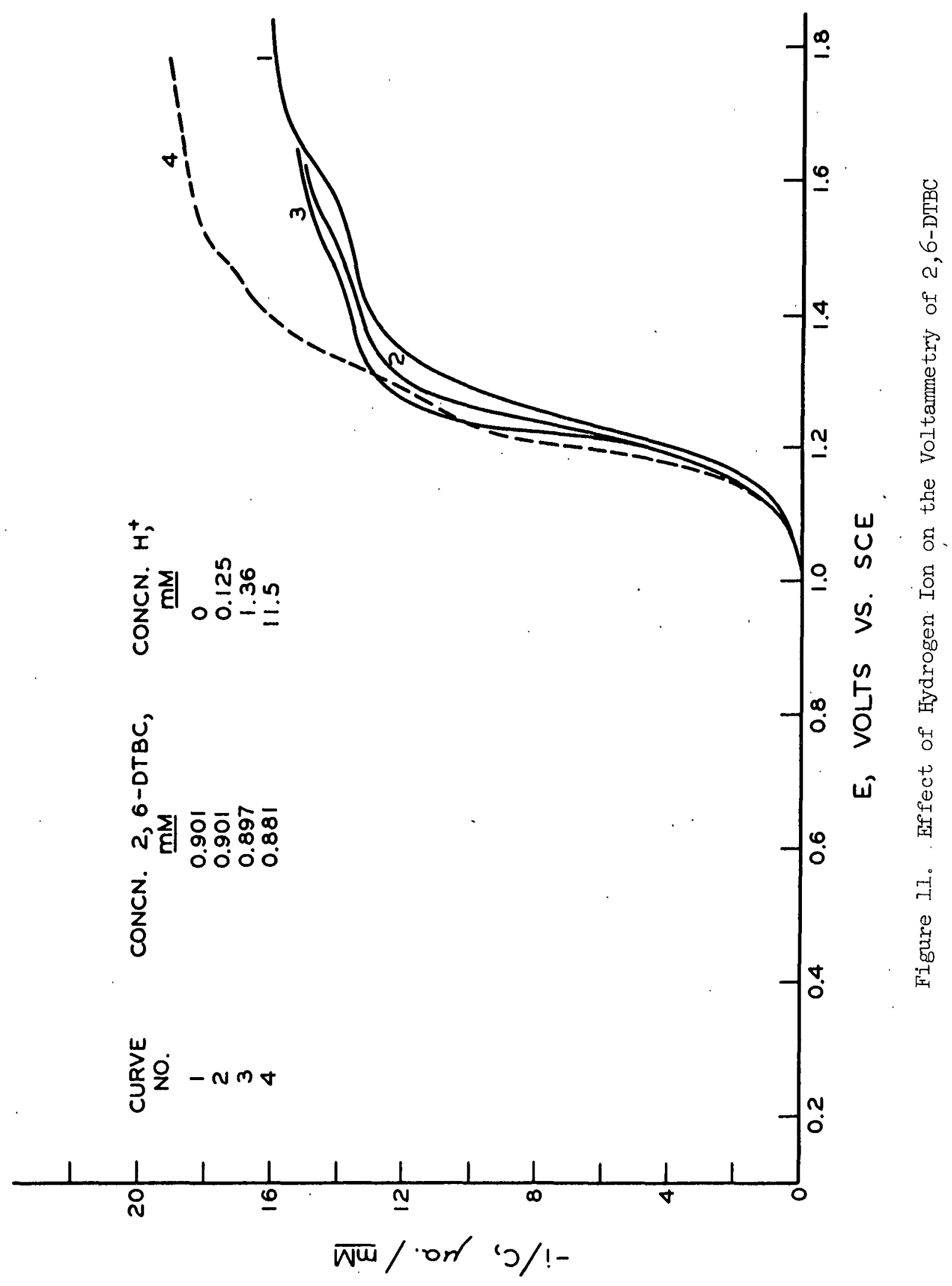




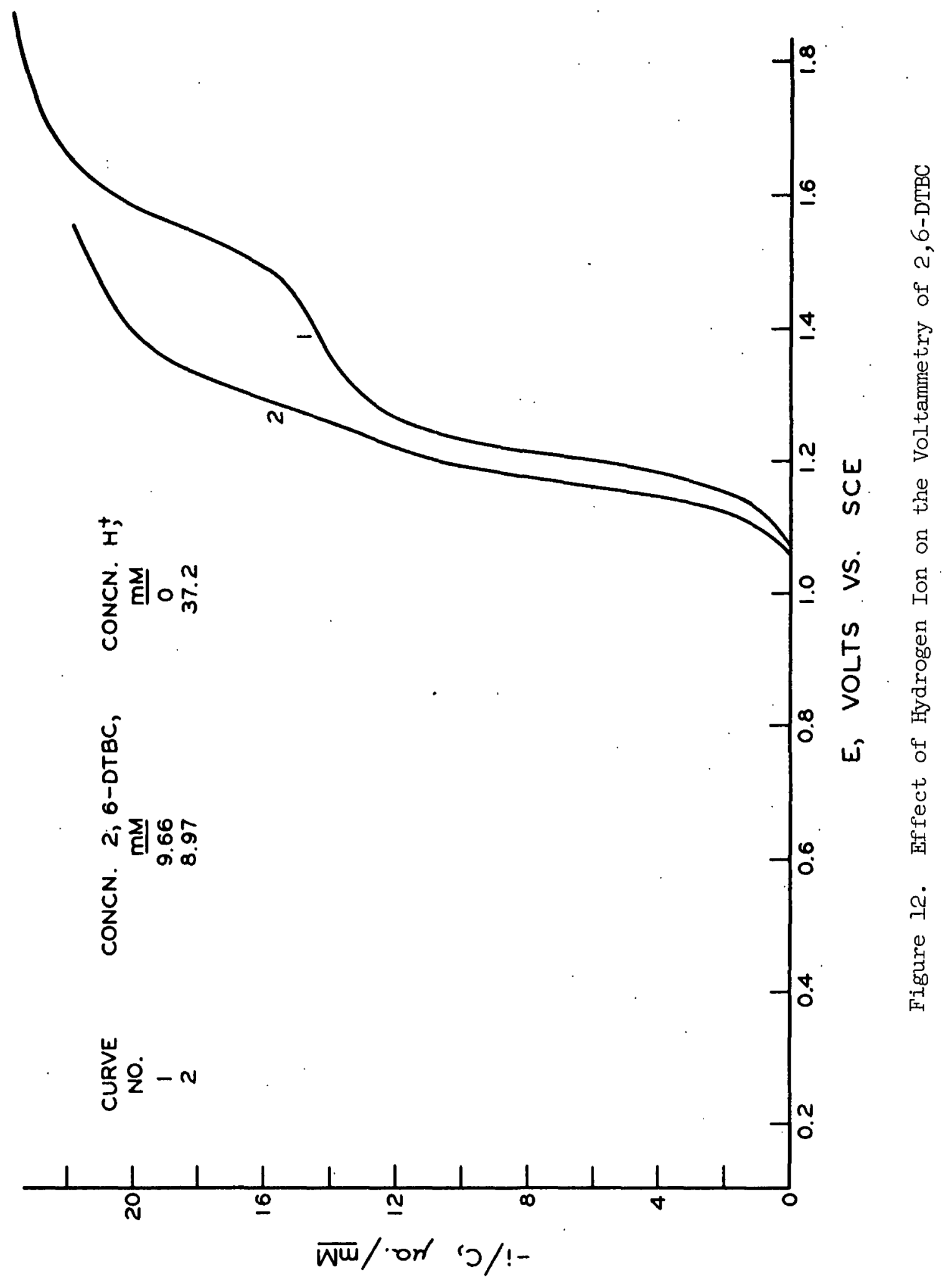



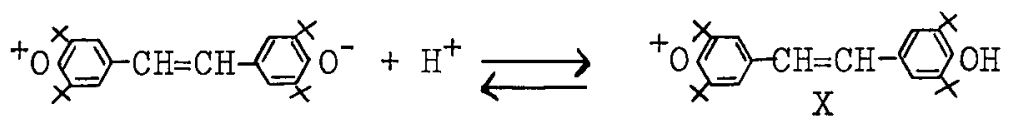

The dihydrostilbene (I) at a concentration level of $0.814 \mathrm{mM}$ shows a numerical increase of $12 \%$ in $\underline{i}_{\underline{D}} / \underline{C}$ for the first wave and a slight numerical decrease in ${ }_{\underline{1}}$ D $\underline{C}$ for the second wave in the presence of excess perchloric acid. This effect, though small, would be expected from the behavior of the stilbenequinone (III) which is the anticipated product from the electrode reaction of the dihydrostilbene.

The stilbene (II) displayed reduced wave heights in the presence of excess perchloric acid. This behavior might be explained by addition of hydrogen ion to the double bond to form an electrochemically inactive species.

The results of these experiments have demonstrated that unless the hydrogen ion is consumed as it is liberated in the electrode reaction and the following processes, destructive oxidation of the products of reaction may be expected in an electrochemical oxidation of 2,6-DTBC.

\section{BUFFERED SOLUTIONS}

The undesirable effect of acid on the voltammetric behavior of 2,6-DTBC and its expected oxidation products made it desirable to develop a suitable "buffer" system for use in acetonitrile. Three buffers were evaluated, pyridine, tetraethylammonium nitrate-nitric acid, and tetraethylammonium acetate-acetic acid. The pyridine (0.IM) produced the unique peaked waves typical of all of the phenols studied in this system, and discussed at greater length in the Appendix.

The report by Kolthoff, et al. (36) that nitric acid behaves as a weak acid in acetonitrile made the tetraethylammonium nitrate-nitric acid system seem like an attractive possibility for buffering of an acetonitrile solution. The addition 
of tetraethylammonium nitrate $(2 \mathrm{mM})$ in a solution which was $1.05 \mathrm{mM}$ in 2,6-DTBC reduced $\underline{i}_{\underline{D}} / \underline{C}$ to a value of $-10.4 \mu \mathrm{a} . / \mathrm{mM}$, close to the value for a two-electron reaction. This may have been caused by addition of the nitrate ion to the four position of the phenoxonium ion to form a cyclohexadienone structure. The small second wave of 2,6-DTBC was eliminated, and the half-wave potential of the main wave was shifted to 1.08 volts. When hydrogen ion (as perchloric acid in acetic acid) was added to this solution at the level of $\mathrm{ImM}$ the half-wave potential was lowered further to 0.97 volt and the wave height was decreased $11 \%$. Further addition of hydrogen ion gave a rapid reduction in wave height until at a concentration level of $24 \underline{\mathrm{mM}}$ the wave of $2,6-\mathrm{DTBC}$ was completely eliminated. Since most of the hydrogen ion added to this solution would combine with the nitrate ion as nitric acid, it is probable that a nitration of 2,6-DTBC occurred to give the 4-nitrocyclohexadienone. This is a well-known reaction in other polar solvent systems (49, 66). Because of this side reaction the tetraethylammonium nitratenitric acid system was unsuitable as a buffering agent.

The use of a tetraethylammonium acetate-acetic acid buffer was investigated in a solution which was approximately $0.1 \mathrm{M}$ in acetate ion and $0.05 \mathrm{M}$ in acetic acid. In this solution 2,6-DTBC (1.05M) exhibited a long drawn out prewave which, as will be discussed later, is typical behavior in the presence of a strong base. Since acetic acid in acetonitrile is an extremely weak acid, it follows that its conjugate base, the acetate anion, will be relatively strong, and therefore, the observed behavior is to be expected. The main wave of 2,6-DTBC, which occurred at 1.35 volts under these conditions, was merged with the decomposition wave for the acetate anion. Current time curves at constant potential on the shoulder of this wave revealed abnormal current decay behavior. Thus, each of the three buffers investigated was unsatisfactory. 
EFFECT OF WATER AND METHANOL

The effects of methanol and water on the voltammetric behavior of 2,6-DTBC in acetonitrile are of particular interest because both of these reagents were present during the electrolysis which is discussed in the next section. The addition of 3.7 to $3.8 \%$ water or methanol to a test solution containing 2,6-DTBC (0.5 to $10 \mathrm{mM}$ ) completely removed the second wave, indicating a change in the over-all reaction mechanism. The half-wave potential was shifted to a less positive value (1.01 to 1.05 volts). Values of $\underline{i}_{\underline{D}} / \underline{C}$ ranged from -11.7 to -13.0 (average -12.4 ) $\mu a . / \mathrm{mM}$, approximately a $12 \%$ decrease below the average in this concentration range in the absence of water or methanol.

A pronounced peaking was noted in the wave for 2,6-DTBC at a concentration level of $10 \mathrm{mM}$ in the presence of $3.8 \%$ water, but this peaking was not observed at lower levels of concentration $(0.5$ to $1.5 \mathrm{~mm})$. The peaks were evidently not the result of a polymeric film because the curve could be retraced after simply treating the electrode cathodically at -0.5 volt. No peaks were observed for 2,6-DTBC even at a concentration level of $10 \mathrm{mM}$ with $3.8 \%$ methanol in the test solution.

Assuming that the lowest numerical value of $\underline{i}_{\underline{D}} / \underline{C}$ from Fig。 $9(-9.6 \mu \mathrm{a} . / \underline{\mathrm{mM}})$ represents a two-electron transfer, an $\underline{n}$ value of 2.58 would be indicated for the electrode reaction of $2,6-D T B C$ in the presence of $3.8 \%$ water or methanol. Since a nonintegral $\underline{n}$ value for a single electrode reaction is an impossibility, this calculated value must represent a summation of two or more different electrode processes. A logical explanation is that the primary electrode process gives the mesomeric phenoxonium ion, XI, according to Equation (15), and that part of this reacts with the water or methanol by Equation (15a) to give the cyclohexadienone, XII, which is electrochemically inactive. The other part of the phenoxonium ion, XI, rearranges according to Equation (16) to give the benzyl carbonium ion, XIII, 
and adds water or methanol to form the alcohol or ether, XIV, by Equation (16a). The product XIV can undergo electrochemical oxidation by Equation (16b) at the same or a lower potential than the main wave for 2,6-DTBC to form the phenoxonium ion, XV. This product can in turn give a cyclohexadienone, XVI, by Equation (16c) or rearrange and add water or methanol to give an acetal or an aldehyde by Equations (16a et seq.).
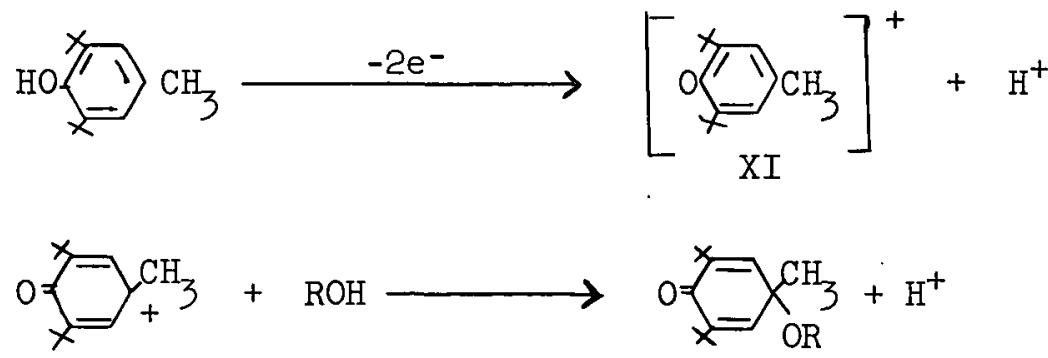

$\mathrm{XI}$

XII

$=\underset{x=\mathrm{CH}_{3} \rightleftarrows \mathrm{HO}}{\longrightarrow} \underset{\mathrm{XIII}}{\mathrm{C}}$

${ }_{x=\mathrm{CH}_{2}}^{+}+\mathrm{ROH} \longrightarrow{ }_{\mathrm{XIV}}^{\mathrm{H}} \mathrm{CH}_{2} \mathrm{OR}+\mathrm{H}^{+}$

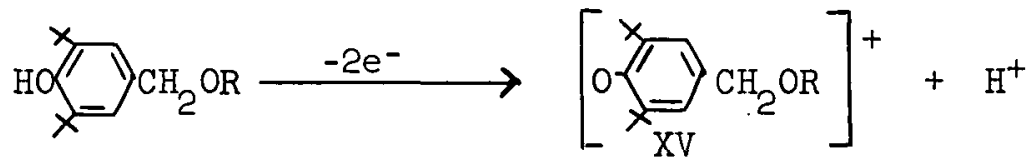

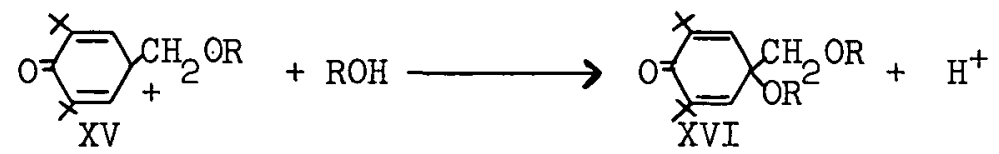

$x_{x=}^{x} \mathrm{CH}_{+2 \mathrm{OR}} \rightleftarrows \mathrm{HO}_{\mathrm{CHOR}}^{+}$

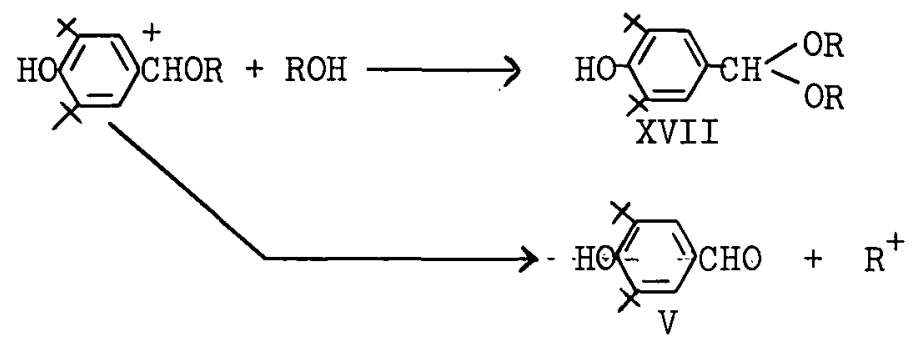


Two voltammetric observations agree with the reaction sequence of Equations (15) and (16): First, the cyclohexadienone, $\mathrm{XII}, \mathrm{R}=\mathrm{CH}_{3}$, is electrochemically inactive within the anodic range of acetonitrile with tetraethylammonium perchlorate as a supporting electrolyte. Although the hydroxy analog has not been investigated in this study, it is to be expected that it would show the same electrochemical behavior. Second, the alcohol, XIV, R=H, gives a wave at about the same potential as 2,6-DTBC (see Table V). It is expected that the methyl ether of this alcohol would give essentially the same half-wave potential because this small change in structure would have little effect on the electron density of the molecule.

In the reaction scheme postulated by Equations (16) it will be noted that the first step is the rearrangement of the phenoxonium ion to the benzyl carbonium ion. Recalling the discussion on page 5l, this shift should be catalyzed by the presence of hydrogen ion. Therefore, as the concentration of 2,6-DTBC is increased and more hydrogen ion is generated, it should be expected that this shift would be facilitated and as a result that Equations (16) would account for more of the reaction

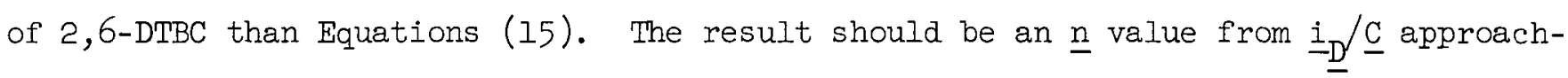
ing 4 at high levels of concentration. Over the concentration range studied, however, (0.5 to $10 \underline{\mathrm{mM}}$ ) no significant trend of $\underline{\underline{i}}_{\underline{D}} / \underline{C}$ was apparent with either water or methanol. The explanation for this behavior may lie in the ability of water or methanol to solvate the liberated hydrogen ion, thus decreasing its activity and therefore its relative influence on the rearrangement. This view is supported by the report of Kolthoff, et al. (36) that water is a "fairly strong base" in acetonitrile. It therefore appears that water or methanol acts to a degree as a "buffering agent" in the vicinity of the electrode, as well as participating in the electrode reaction. 
From the apparent $\underline{n}$ value of 2.58 for the electrode reaction of $2,6-D T B C$ in the presence of $3.8 \%$ water or methanol it is possible to calculate the percentage of products to be expected from Equations (15) and (16), 'respectively, assuming that these are the only reactions which occur at the electrode. Thus, if $x$ represents the fraction of $2,6-D T B C$ reacting according to the two-electron process of Equation (15), and $1-\underline{x}$ the fraction reacting by the four-electron process of Equation (16), the net $\underline{n}$ value will be given as $\underline{n}=2 \underline{x}+4(1-\underline{x})=2.58$. Thus, $\underline{x}=0.71$. In other words, it would be predicted that if a controlled potential electrolysis in the presence of excess water or methanol were carried out on the diffusion plateau, that $71 \%$ of the $2,6-\mathrm{DTBC}$ would react to form the cyclohexadienone, XII, and that in the other $29 \%$ the para methyl group would undergo successive oxidations to give an acetal or an aldehyde.

\section{ELECTROLYSIS}

Although the results in the previous section indicated that water or methanol in the solution acted as a partial buffer in the vicinity of the electrode so that no appreciable trend of $\underline{\underline{i}}_{\underline{D}} / \underline{C}$ with concentration was observed, the cyclohexadienone (VII) at a concentration level of $0.159 \mathrm{mM}$ was known to give a voltammetric wave $\left(\underline{E}_{1 / 2}=1.35 \mathrm{v} ., \underline{i}_{\underline{D}} / \underline{C}=-8.8 \mu \mathrm{\mu} . / \underline{\mathrm{mM}}\right)$ in the presence of excess hydrogen ion $(8.1 \mathrm{mM})$. The origin of this wave was unknown, but its presence indicated that the primary product of electrolysis would be destroyed if the electrolysis were conducted in a solution where the liberated hydrogen ion was not neutralized. On the other hand, the presence of excess strong base could not be tolerated since this would cause ionization of the phenol, and consequently a free radical reaction as discussed in a later section. Since no satisfactory buffer system had been devised, an alternative approach was indicated. 
For each electron removed from a neutral organic molecule in an anodic reaction there is left in solution one positively charged ion, either a hydrogen ion or a carbonium ion. The carbonium ion is in most cases very unstable, and may be expected to combine rapidly with other molecules in the solution to give a neutral molecule and a hydrogen ion in reactions of the sort which have been described in the previous pages. Ultimately, therefore, there should be a net generation of one hydrogen ion in the solution for every electron which is transferred at the electrode; i.e., one equivalent of hydrogen ion per Faraday of electricity. This principle, once conceived, offered a practical way out of the buffering dilemma. While the electrolysis was being conducted a base could be added continuously at a rate which was exactly equivalent to the current flowing.

An electrolysis by this method was conducted on a $10^{-2}$ M solution of 2,6-DTBC in acetonitrile with $0.5 \mathrm{M}$ sodium perchlorate as a supporting electrolyte. To minimize any overconcentration of hydrogen ion in the vicinity of the electrode the electrolysis was conducted at a constant low current density of $1 \mathrm{ma} . / \mathrm{sq} . \mathrm{cm}$., corresponding approximately to the current density. at a concentration of $0.5 \mathrm{mM}$ in the voltammetric studies.

The base added was tetraethylammonium hydroxide dissolved in methanol. The solution originally contained no methanol, but the rate of addition was such that by the time electrolysis was carried to $1 \%$ completion the methanol concentration was $16 \mathrm{mM}$, and at the end of the electrolysis the methanol concentration should have been $1600 \mathrm{mM}$. Originally, the solution was probably only 1 to $2 \mathrm{mM}$ in water, but as the electrolysis progressed more water was presumably formed at the rate of one equivalent per Faraday, and therefore its concentration at the end of the electrolysis would have been in the order of $40 \mathrm{mM}$. 
The plot of anode potential vs. time for this electrolysis in Fig. 13 shows a small decrease in potential during the first 600 minutes which was expected from the observed shift in half-wave potential for 2,6-DTBC to a less positive value in a solution containing water or methanol. For the next 700 minutes the potential remained relatively steady and then for 700 minutes it rose gradually as is expected in an electrolysis at constant current. Then for a period of 500 minutes the anode potential varied very erratically and some extremely low potentials ( $<0.4$ volt) were observed. This behavior is significant, indicating the formation of electrolysis products which had a very low half-wave potential. The anode potential rose rather sharply during the last part of the electrolysis to a final value of 1.55 volts. At the low current density the original 2,6-DTBC should have been more than 95\% consumed for the potential to reach this level.

The coulometric $\underline{n}$ value calculated for this electrolysis was 3.45 . By titration the anolyte was shown to contain excess base equivalent to $3 \%$ of the total hydroxide ion added during the electrolysis. A crude product was separated from the anolyte in 103\% yield based on the starting 2,6-DTBC.

2,6-Di-tert-butyl-4-methyl-4-methoxycyclohexadienone, VII, was separated from the crude product and positively identified. By quantitative gas chromatography this compound was known to represent $65.3 \%$ of the crude product, a remarkably good agreement with the $71 \%$ predicted from the voltammetric studies.

No evidence was found for the aldehyde or acetal predicted as secondary products from this electrolysis. However, another product, indicated as $10.4 \%$ of the crude product by quantitative gas chromatography, was separated in a large enough quantity for an infrared spectrum by collection from the gas chromatograph. Its spectrum was identical with that of 2,6-di-tert-butyl-1,4-benzoquinone. 


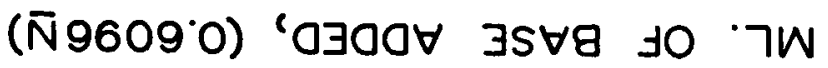

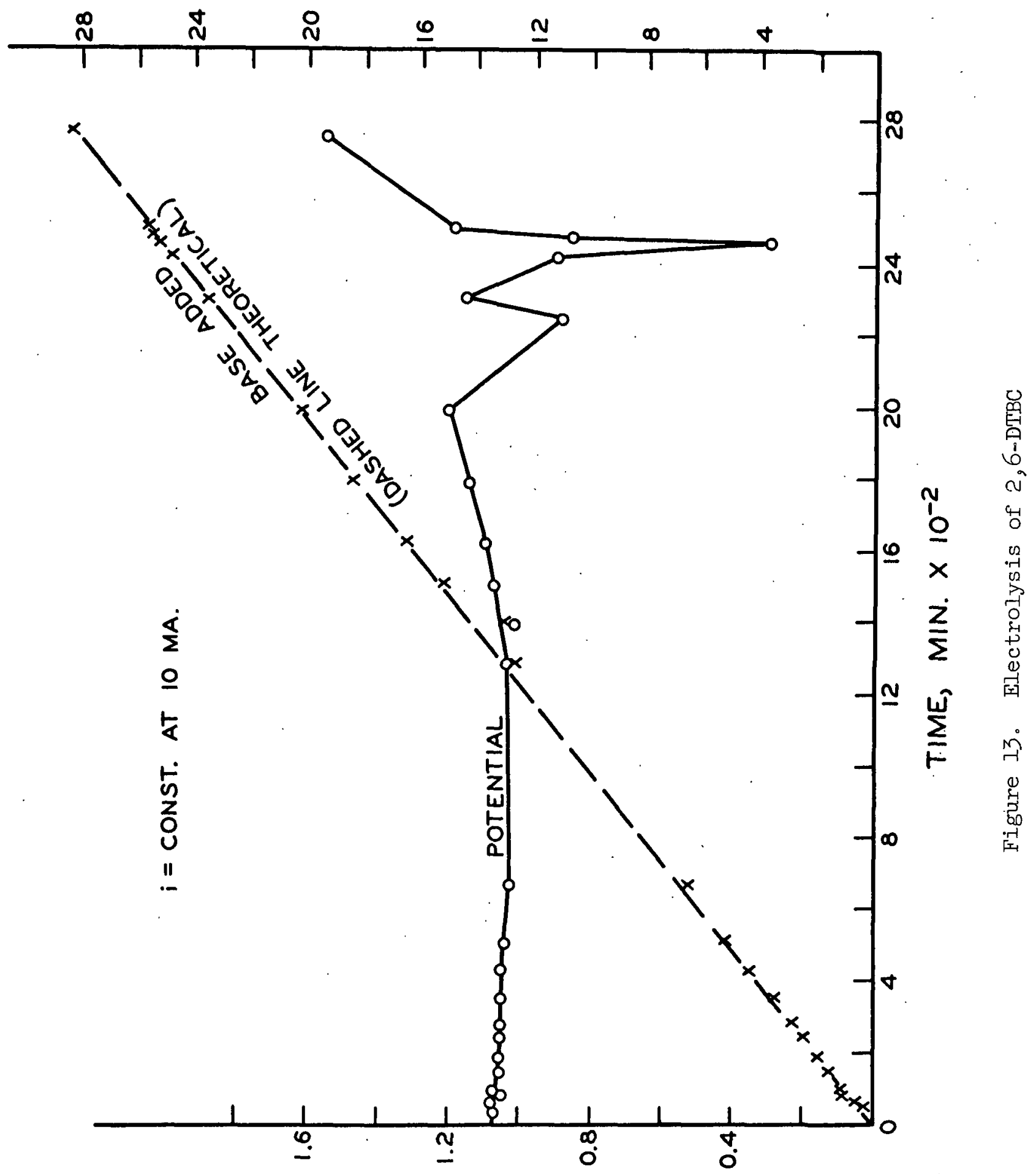

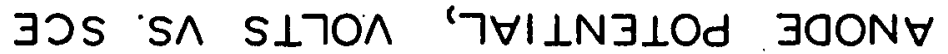


No other products were separated from the reaction mixture. Altogether $83.7 \%$ of the crude product was volatile enough to pass through a Carbowax column on the gas chromatograph at temperatures of 150 to $190^{\circ} \mathrm{C}$. An infrared spectrum carbonyl band at $5.78 \mu$ in the product which would not sublime at 50 to $60^{\circ} \mathrm{C}$. under vacuum (aspirator pressure) could possibly have been caused by an ester of 3,5-di-t-butyl4-hydroxybenzoic acid, which gives a carbonyl band at $5.83 \mu$.

The question arises as to the origin of the 2,6-di-tert-butyl-1,4-benzoquinone. Cook, et al. (49) believe that in the chemical oxidation of 2,6-DTBC this product is formed via the "oxidatively induced decarboxylation of 3,5-di-tert-butyl-4hydroxybenzoic acid" presumably via the corresponding free radical. It would seem reasonable that the electrochemical oxidation should follow a similar path.

The formation of the aldehyde, $V$, is readily explained by the reactions of Equations (16) or by hydrolysis of the acetal, XVII, but oxidation of the aldehyde to the acid, VI, by a two-electron process would not have been possible because this reaction occurs at a potential considerably higher than existed at the anode during the electrolysis (see Table V, p. 49). However, it is known that an excess of base was present in the anode compartment during the latter stages of the electrolysis. This would lead to ionization of some phenols in the reaction mixture. As discussed in more detail in a later section, the phenoxide anion reacts via a one-electron free radical mechanism at a much lower potential than the nonionized phenol. Furthermore, because acetonitrile is a "differentiating" solvent, the aldehyde, $\mathrm{V}$, should be ionized preferentially before the unreacted 2,6-DTBC* A possible reaction sequence for the aldehyde, V, to yield the acid, VI, or an ester,

\footnotetext{
*Acetonitrile is a differentiating solvent because phenols with slightly different acidities in water have greatly different acidities in acetonitrile. This is demonstrated by the voltammetric studies on vanillin and dehydrodivanillin in the presence of hydroxyl ion, p. 87.
} 
XVIII, by a one-electron primary reaction, but an over-all transfer of two electrons is given by Equations (17). The occurrence of XVIII in the reaction mixture would explain the unidentified carbonyl band at $5.78 \mu$ in the infrared spectrum of the crude product of electrolysis.
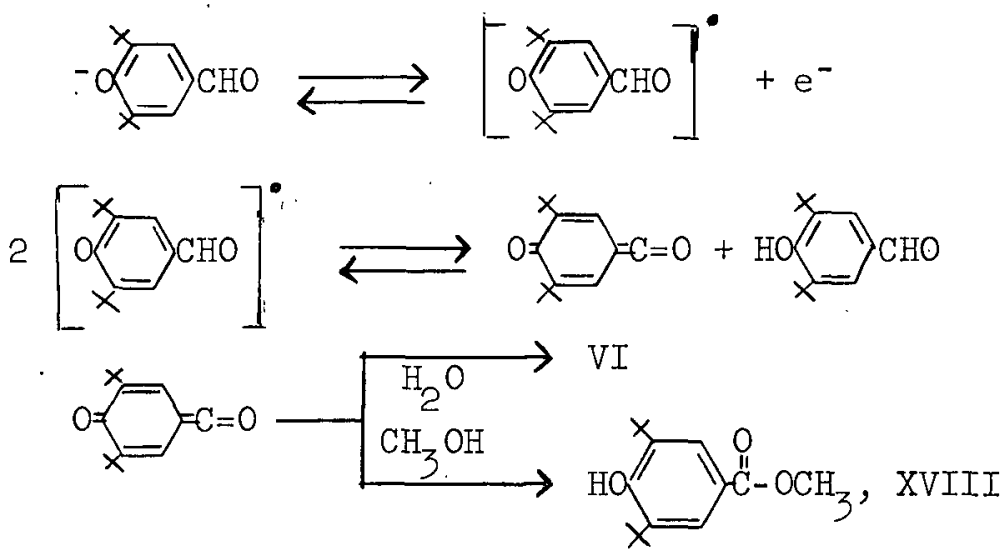

The acid, VI, in an ionized form could react at the electrode in the manner of "Equation (18). This reaction is evidently the first step in the Kolbe synthesis (4). Geske has observed further that the acetate ion reacts in a one-electron reaction at 1.58 to 1.63 volts in acetonitrile (26) at concentrations from 0.3 to 4. OmM. In the present study a brief investigation of the acid, VI, $(0.586 \mathrm{mM})$ in the presence of an equivalent amount of tetraethylammonium hydroxide and $3.8 \%$ methanol showed a one-electron reaction $(\underline{\underline{i}} \underline{\underline{C}} \underline{C}=-4.8 \mu \mathrm{a} . / \underline{\mathrm{mM}})$. The wave was very drawn out, extending over the range from 0 to 0.91 volt, a behavior which is readily explained by partial ionization of the phenolic group. Upon addition of a second equivalent of tetraethylammonium hydroxide the one-electron wave for the phenoxide anion $(\underline{\underline{D}} / \underline{C}=-4.6 \mu \mathrm{a} . / \underline{\mathrm{mM}}$ ) appeared at -0.14 volt. These experiments establish the single electron transfer of Equation (18). Whether the phenoxide group or the carboxylate group is attacked first would make no difference since these are resonance forms of the same free radical. A decarboxylation reaction to yield a new free radical can occur by Equation (19). 


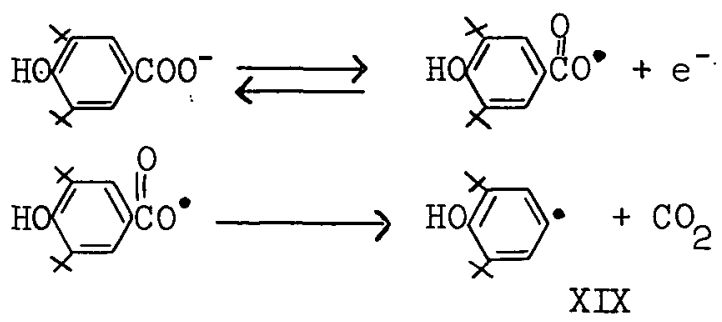

The manner of reaction of the radical, XIX, is uncertain. It would probably rearrange to form the phenoxy free radical which Cook (49) suggests forms 2,6-ditert-butyl-1,4-benzoquinone by reacting with oxygen. In the present case, however, it is presumed that the solution was oxygen free, since a stream of nitrogen was bubbling through the cell throughout the electrolysis. Therefore, it would seem that water or methanol from the reaction mixture must have combined with the radical, XIX, in an over-all reaction which involved the removal of another electron in the manner of Equation (20). The hydroquinone, $\mathrm{XX}$, could then react in a twoelectron reaction to give the quinone, VIII, by a two-electron transfer, Equation (21).

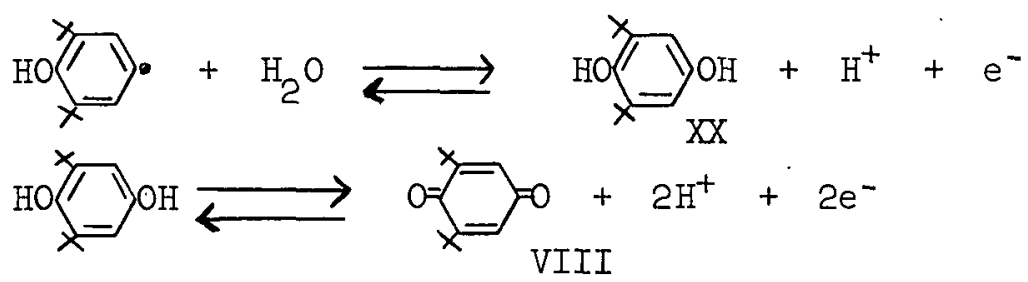

The over-all reaction represented by Equations (16) through (21) is a tenelectron process. This does not represent a proven mechanism, but merely an attempt to explain logically the occurrence of the quinone, VIII, in the crude product of electrolysis. It is, however, quite certain that free radical mechanisms were involved because of the extremely low potentials observed during the later part of the electrolysis. Furthermore, the proposed mechanism could account for the observed coulometric $\underline{n}$ value. Thus, if the cyclohexadienone, VII, is the result of a two-electron transfer, the quinone, VIII, the result of a ten-electron transfer, 
and the unidentified portion (24.3\%) of the crude product is assumed* to have represented an average five-electron transfer, the calculated coulometric $\underline{n}$ value for the electrolysis is 3.55 in close agreement with the observed value of 3.45 .

The most significant result of this electrolysis is the relatively high yield of the cyclohexadienone, VII. Since, in chemical oxidations of 2,6-DTBC, this type of product results from the use of ionic reagents such as bromine or nitric acid in polar media (48, 68, 69), the occurrence as an electrolysis product is confirming evidence for the phenoxonium ion mechanism in the electrode reaction of the nonionized phenol.

\section{DISCUSSION}

The electrochemical reactions of 2,6-DTBC in acetonitrile which have been indicated voltammetrically are summarized in Fig. 14. Each of the electrode reactions in the figure should occur at the same or a lower potential than the primary reaction of $2,6-\mathrm{DTBC}$ so it is quite certain that if the indicated initial products are formed in an electrolysis at constant potential, the secondary electrode reactions will also occur. The primary electrode reaction is the loss of a hydrogen ion and two electrons to form a mesomeric phenoxonium ion.

\footnotetext{
*There are three intuitive reasons for assuming an average five-electron transfer in the unidentified portion of the crude product. First, at the low current density used, the original 2,6-DTBC should have been nearly all consumed, i.e., at least a two-electron transfer. Second, the unidentified carbonyl band in the infrared spectrum of the crude product at $5.78 \mu$ indicated, the possibility that an ester of 3,5di-tert-butyl-4-hydroxybenzoic acid was present. This would be the result of an over-all six-electron transfer. Third, the identified portion of the crude product $(75 \%)$ did not indicate that extreme degradation reactions, representing more than a ten-electron transfer, had occurred. These factors, together with the correlations on $\mathrm{p} .72$ et seq., when viewed with the objective of determining what reactions could have occurred, indicate that the average electron transfer should have been in the range from two to ten.
} 


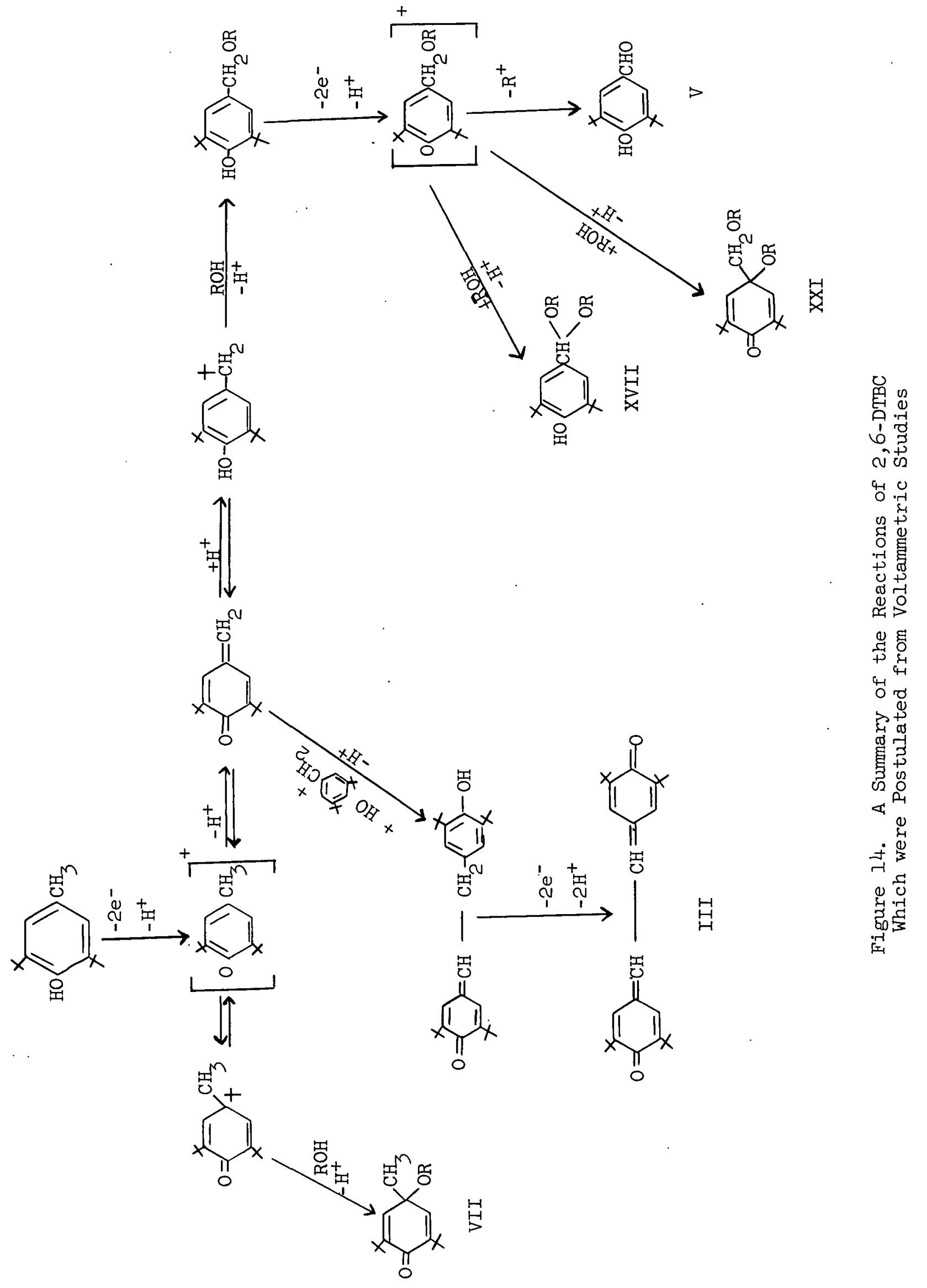


If no nucleophilic reagents are present in the reaction mixture it is believed that the phenoxonium ion stabilizes itself by dimerization through the para-methyl group and the loss of hydrogen ions. The dimeric product so obtained can be further oxidized to a stilbenequinone. Because of difficulties in providing a suitable buffer for the reaction mixture, this reaction sequence was not verified by coulometry and controlled potential electrolysis. It would be of considerable interest to investigate this reaction further, because there appear to be very few cases mentioned in the literature of phenolic dimerizations or polymerizations occurring by ionic processes. The electrochemical technique offers the unique advantage of providing direct evidence that the phenoxonium ion intermediate is indeed formed as the first reaction. Two possibilities suggest themselves for further study. First, it may be possible to conduct an electrolysis by the method used in the present study, only using a weak base which will not react with acetonitrile. The base, which it is presumed would act as a proton acceptor, could then be dissolved directly in acetonitrile rather than in a nucleophilic reagent. Second, if a phenol which has blocking groups other than the "hindering" tertiary butyl groups, in two or more of the reactive ortho- and para-positions, it is likely that the intermediate phenoxionium ion would react preferentially in a dimerization-reaction rather than in an addition reaction with methanol or water. In this case a strong base could'be'used in the manner' of the present study.

The addition of methanol to the phenoxonium ion of 2,6-DTBC was confirmed by a large-scale electrolysis. The hydrogen ion liberated in the reaction was neutralized by the addition of methanolic tetraethylammonium hydroxide to the anode compartment at the rate of one equivalent per Faraday of electricity. The yield (65\%) of the cyclohexadienone, VII, agreed very well with the yield predicted by voltammetry (71\%). This is an example of an anodic substitution reaction. It is of interest 
to note that the reported anodic substitution of phenolic molecules with the thiocyanate group occurred in a strong acid solution ( $\underline{4}, \underline{5})$. . It may be expected that under these conditions the nonionized phenol would react at a lower potential than the phenoxide anion*--hence a phenoxonium ion mechanism would be anticipated from the results of the present study. It should be possible to add nucleophilic reagents other, than methanol or water to phenoxonium ions generated from phenols in acetonitrile. The study of such reactions would be a logical extension of the present work.

Neither the aldehyde, V, the acetal,.XVII, nor the cyclohexadienone, XXI, in the reaction scheme of Fig. 14 were isolated from the crude product of the 2,6-DTBC electrolysis, but 2,6-di-tert-butyl-1,4-benzoquinone was indicated in 10.4\% yield. It is suggested that this product could have occurred via a stepwise oxidation of the methyl group of 2,6-DTBC with a decarboxylation of 3,5-di-tert-butyl-4-hydroxybenzoic acid in the over-all reaction of Equation (18). An exact mechanism cannot be detailed for this reaction, but it seems certain that free radical processes of the intermediate phenoxide anions were involved.

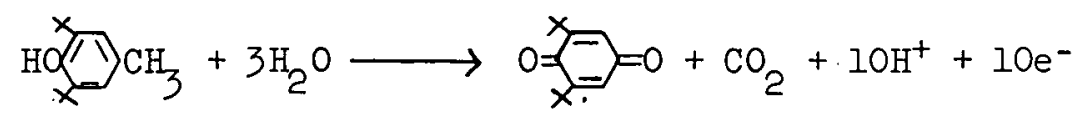

The progressive oxidation of a side chain on a phenolic molecule is of considerable interest to the wood chemist, and is deserving of further study with extension to more complicated side chains.

\section{OTHER HINDERED PHENOLS}

In Table VI data are presented for hydroquinone, 2,6-DTBC, 2, 4,6-tri-tertbutylphenol, and 2,6-di-tert-butylphenol. There are two differences between the *A discussion of the electrode reactions of phenols as a function of $\mathrm{pH}$ in aqueous
systems will be found on page 99 . 
TABLE VI

VOLTAMMETRY OF HINDERED PHENOIS

(Electrode No. 8, reduced surface) ${ }^{\mathrm{a}}$

Compound

Conen., $\underline{\mathrm{mM}}$

0.0285

0.0570

0.113

0.0266

0.0235

43.9

36.7

38.4

0.0939

0.0472

2,6-Di-tert-

butylphenol
0.0285
46.9
1.47
43.4
46.3
0.0570
1.45
0.51
0.114
1.49
0.49
0.48

$\underline{E}_{1 / 2}$

volts vs. sic.e.

$\beta \underline{n}_{\beta}$

$0.98 \quad 0.58$

$0.99 \quad 0.64$

$1.00 \quad 0.65$

1.28

0.48

0.60

an these experiments cell resistance was measured with a leeds \& Northrup bridge. Because of the low current level in the concentration range covered by this table, this would introduce no significant error into the calculated results. 
experimental conditions for these data and the experimental conditions for the voltammetric data in the previous section. The first is that $0.1 \mathrm{M}$ instead of 0.5M tetraethylammonium perchlorate was used as a supporting electrolyte. This is not believed to have resulted in any significant change in the observed results. The second is that an oxidized rather than a reduced platinum electrode was used (Electrode No. 8). As pointed out previously an oxidized surface results generally in a higher half-wave potential than a reduced surface. Thus, $E_{1 / 2}$ for $2,6-D T B C$ in Table VI is 1.28 volts as compared to 1.20 volts in Table IV. Similarly, hydroquinone was found to have a half-wave potential of 0.89 volt on an electrode reduced with acidic ferrous ammonium sulfate as compared with 0.99 volt on an oxidized surface in Table VI:

The compounds in Table VI all give diffusion currents in the range from -35 to $-50 \mu \mathrm{a} / \mathrm{mM}$ indicating by comparison with 2,6-DTBC a primary two-electron electrode reaction. The differences observed between compounds may be attributed to differences in molecular weight as well as secondary reactions of the sort described in the previous section for 2,6-DTBC. The data of Table V, p. 49, on the oxidation products of 2,6-DTBC indicates that these hindered phenols also were oxidized by a primary two-electron electrode reaction. An exception is, as might be expected, the dimer, I, which evidently undergoes a four-electron transfer.

\section{CORRELATION OF HALF-WAVE POTENTIAIS}

Since a number of phenols of the general formula Ho $R$ have been investigated in the course of this study, it was logical to attempt a correlation of halfwave potentials using the Hammett equation (lㅣ) which.in the case of a totally irreversible electrode reaction at $25^{\circ} \mathrm{C}$. may be stated*

*The derivation of this equation is given in the Appendix. 


$$
\underline{E}_{1 / 2}-\underline{E}_{1 / 2}^{0}=-\frac{0.0591}{\beta \underline{n}_{\beta}} \sigma \rho
$$

where

$\begin{aligned} E_{1 / 2}^{0}= & \text { half-wave potential of the compound with hydrogen in the } \\ & \text { para position }\end{aligned}$ $E_{1 / 2}=$ half-wave potential of a compound with another substituent
in the para position

$\sigma=$ Hammett substituent constant

$\rho \quad=$ Hammett reaction constant.

In accordance with Equation (19), a plot of " $E_{1 / 2}$ vs. $\sigma$ in Fig. 15 is a straightline function. Actually, two parallel straight lines are obtained, one for an oxidized and one for a reduced platinum surface. The correlation fits within the precision of the experimental data. The average value of $\beta \underline{n}_{\beta}$ from Table VI is 0.5 , and the slope of the lines in Fig. 15 is 1.16, so from Equation (19) the value of $\rho$ is calculated as -9.8 .

In the correlation of Fig. 15 the $\sigma$ values for para aldehyde and carboxyl groups fitted the observed data rather than the $\sigma^{c}$ values which are normally used for these groups for reactions involving the phenolic hydroxyl (ㄹ) ). The best explanation for this is that the phenolic hydroxyl is not directly involved in the electrode reaction. Two other factors argue that this is the case. First, in the nonionized phenol the centers of electron density are in the positions ortho and para to the phenolic hydroxyl, and one should expect that the point of electrode attack should be at one of these electron-rich centers. Second, the cyclohexadienone, VII, isolated as a product of electrolysis is also obtained in chemical oxidations with molecular bromine (48), a reagent which evidently reacts with aromatics through the electrophilic attack of the bromonium ion on the aromatic ring (리). 


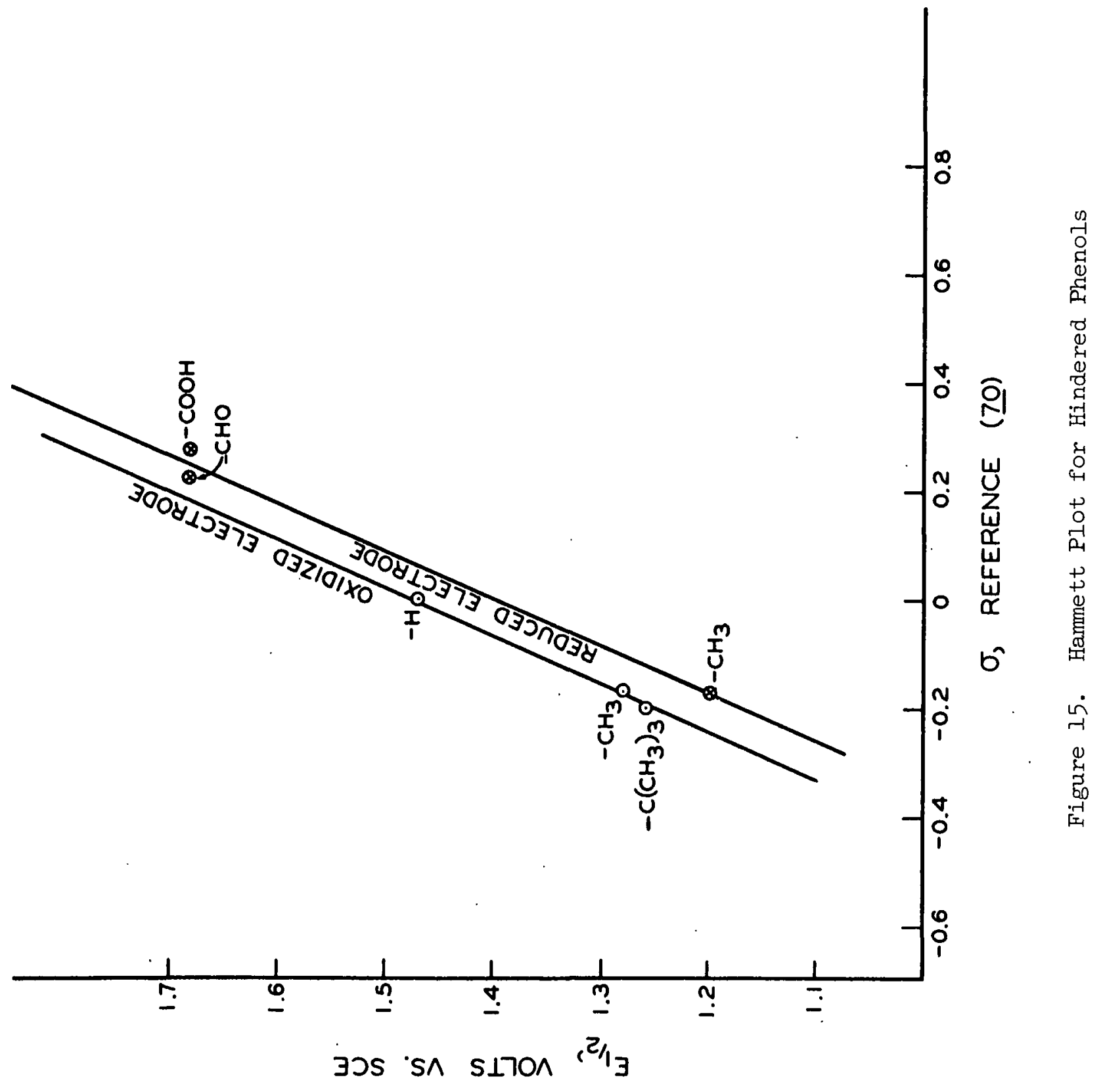


If the hypothesis advanced in the previous paragraph is correct, a correlation similar to that of Fig. 15 should also be applicable to aromatic compounds which do not contain the phenolic hydroxyl. Lund (3I, $\underline{32}$ ) has tabulated voltammetric data in acetonitrile for a few monosubstituted methoxyl compounds which he believes lose two electrons in a primary attack on the aromatic ring. His half-wave potential data are plotted as a function of $\sigma$ in Fig. 16. It was assumed for this plot that the $\sigma$ value for para or meta $-\mathrm{CH}_{2} \mathrm{OH}$ was the same as $\sigma$ for para or meta $-\mathrm{CH}_{3}$, respectively, a reasonable assumption in view of the small difference in $E_{1 / 2}$ for those substituents in Table V. Again the correlation fits within the limits of experimental precision, and the $\sigma$ rather than the $\sigma^{c}$ value fits the para aldehyde group. The value of $\rho$ for this plot is -8.9 assuming that $\beta \underline{n}_{\beta}$ is 0.5 .

At this point one may well ask why the correlations of Fig. 15 and 16 fit so well if the electrode mechanism involves an electrophilic attack on the aromatic nucleus rather than attack on the phenolic hydroxyl or the methoxyl group. The answer to this is that the phenolic hydroxyl or methoxyl is the main polarizing group on the molecule, and that the other substituents may be regarded as adding or subtracting from the $\sigma$ values for this group.

If the electrochemical oxidation involves an electrophilic attack on the aromatic nucleus for both methoxyl and hydroxyl substituents it should be possible to develop a more general correlation using $\sigma^{+}$values for electrophilic substitution (21). An attempt at such a correlation is given in Fig. 17. The values for this plot were calculated in the following manner:

1. It was assumed that in each case the point of attack was. or.tho or para to the hydroxyl: or methoxyl substituent, and that $\sigma^{+}$for a substituent ortho to the point of attack was the same as $\sigma^{+}$for a substituent para to the point of attack.

2. It was assumed that $\sigma^{+}$for a substituent at the site of attack was the same as $\sigma^{+}$for a substituent meta to the site of attack. 


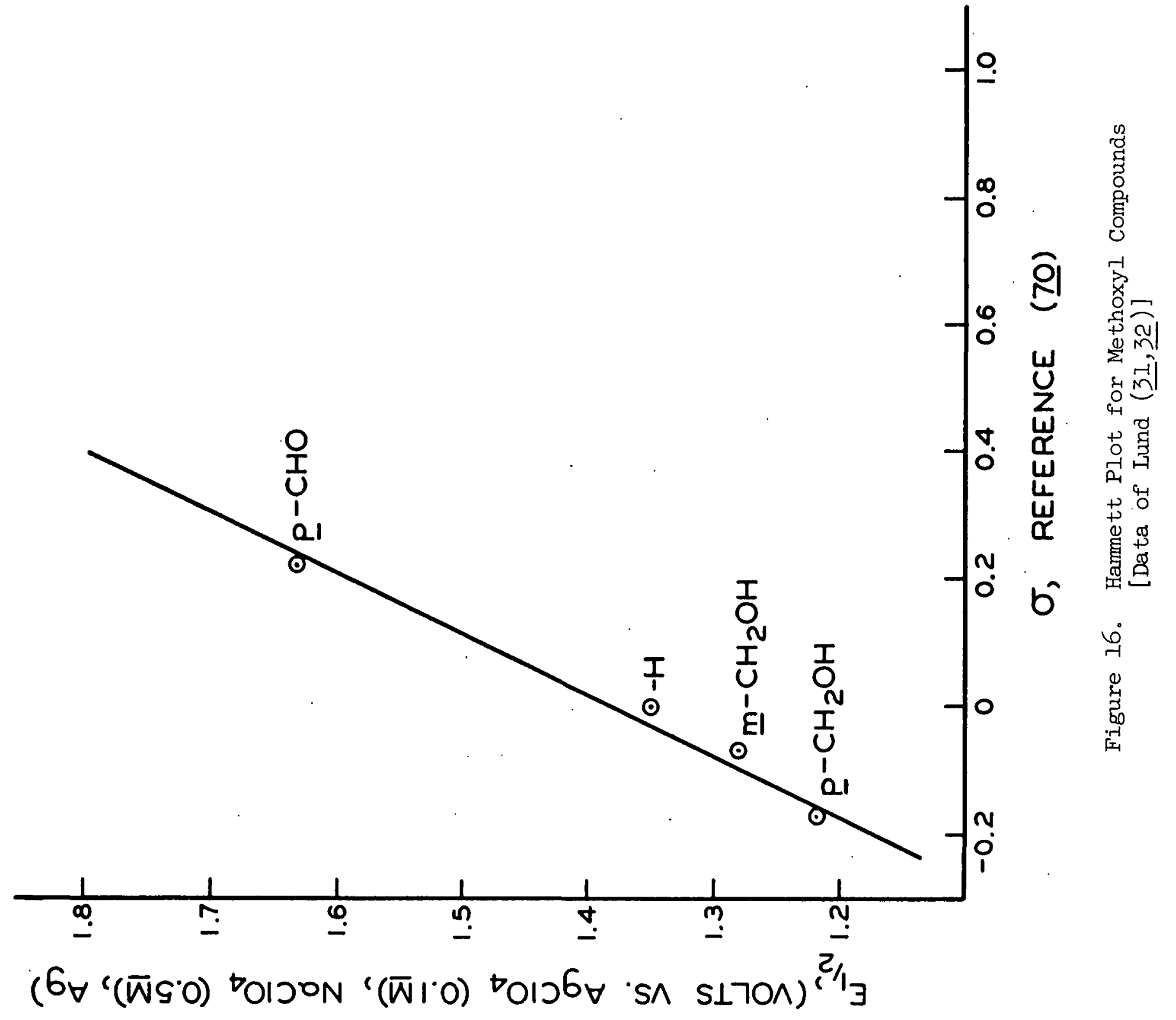




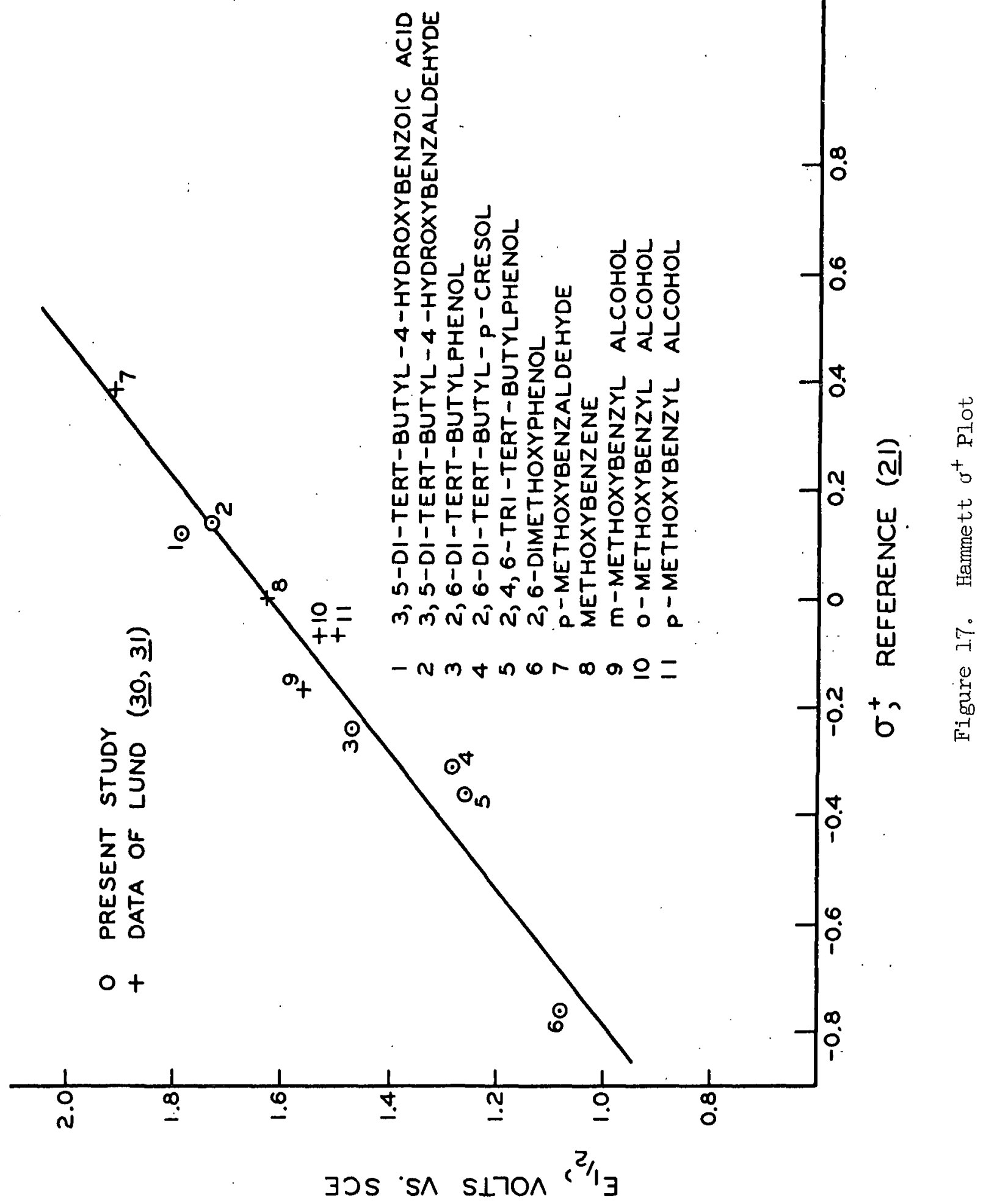


3. The half-wave potential data of Lund vs. the $\mathrm{Ag} / \mathrm{Ag}^{+}$electrode was converted to the s.c.e.-scale by adding a factor of to.30 volt (61).

4. From Lund's discussion of his experimental method it was believed that his platinum electrodes were probably oxidized. Therefore half-wave potentials for compounds of the present study were those for oxidized electrodes as estimated from Fig. 15.

Furthermore, since no $\sigma^{+}$value was available for the para or ortho hydroxyl substituent (lㅣ), this value as well as $\sigma^{+}$for the para or ortho methoxyl substituent was taken as zero. It was expected that, by using this procedure, two parallel lines, one for methoxyl and one for hydroxyl compounds would be obtained. Actually, in Fig. 17 all the points seem to scatter around the same line. Therefore, it appears that there is little difference between $\sigma^{+}$for a hydroxyl and $\sigma^{+}$for a methoxyl substituent. Since $\sigma^{+}$for the para methoxyl group is given as -0.76 (2l), the plot of Fig. 17 is displaced by this amount.

Considering the assumptions which were made in the calculation of $\sigma^{+}$values, the plot of Fig. 17 fits the observed data amazingly well. This is further confirmation of the theory that the electrode reaction involves an electrophilic attack on the aromatic nucleus. It is believed that by a systematic study of other simple aromatic compounds a more precise general correlation can be achieved using adjusted values of $\sigma^{+}$in the manner described for the free radical reaction of the phenoxide anions in later sections of this thesis.

The utility of the correlation in Fig. 17 is demonstrated by the cases of 2,6dimethoxyphenol and vanillin. For the former compound, as discussed in greater detail in the Appendix, an apparent four-electron wave is observed at 1.08 volts, but it is believed that the primary electrode reaction involves two electrons as do the phenols and methoxyl compounds of Fig. 17. The most negative $\sigma^{+}$value for 2,6-dimethoxyphenol, and therefore the highest electron density occurs in the meta 
position to the phenolic hydroxyl. Using a $\sigma^{+}$of -0.76 for the meta position, the half-wave potential for 2,6-dimethoxyphenol fits the correlation of Fig. 17 very well. This may be taken as an indication that the proposed primary electrode reaction is correct. For vanillin, which gives a two-electron wave at 1.43 volts, no $\sigma^{+}$value calculated for an attack on the aromatic nucleus fits the correlation of Fig. 17 at all. This is believed to be an indication that the position of primary electrode attack for vanillin is the carbonyl oxygen.

\section{MECHANISM OF THE ELECTRODE REACTION}

For the reasons advanced in the previous section it is believed that the electrode reaction of the nonionized phenol involves an initial electrophilic attack on the aromatic nucleus at a position either ortho or para to the phenolic hydroxyl. It is known from the value of $\beta \underline{n}_{\beta}$, and the insensitivity of the halfwave potential to added hydrogen ion that the reaction may be classified as totally irreversible. The average value of $\beta \underline{n}_{\beta}$ at low levels of concentration on an oxidized electrode is about 0.5. It is impossible from the evidence at hand to separate $\beta$, the transfer coefficient, from $\mathrm{n}_{\beta}$, the number of electrons transferred in the potential determining step of the electrode process. Lund (31, $\underline{32}$ ) favors the theory that one electron is removed at a time (i.e., $n_{-\beta}=1$ ). The present author, by further analogy with the electrophilic attack of chemical oxidants, favors the simultaneous removal of two electrons. Thus, when the bromonium ion attacks an aromatic ring and is subsequently cleaved as bromide, there has been a simultaneous removal of two electrons from the original molecule.

A schematic representation of this viewpoint for the electrode reaction of a phenol is presented in Fig. 18. In Step I the molecule becomes polarized under the influence of the electric field. As a closer approach to the electrode surface is realized in Step 2, two electrons are attracted from the molecule to the electrode surface with the transitory formation of a bond having some covalent character. 
These electrons are further withdrawn from the molecule until actual cleavage occurs to give the phenoxonium ion and a hydrogen ion.

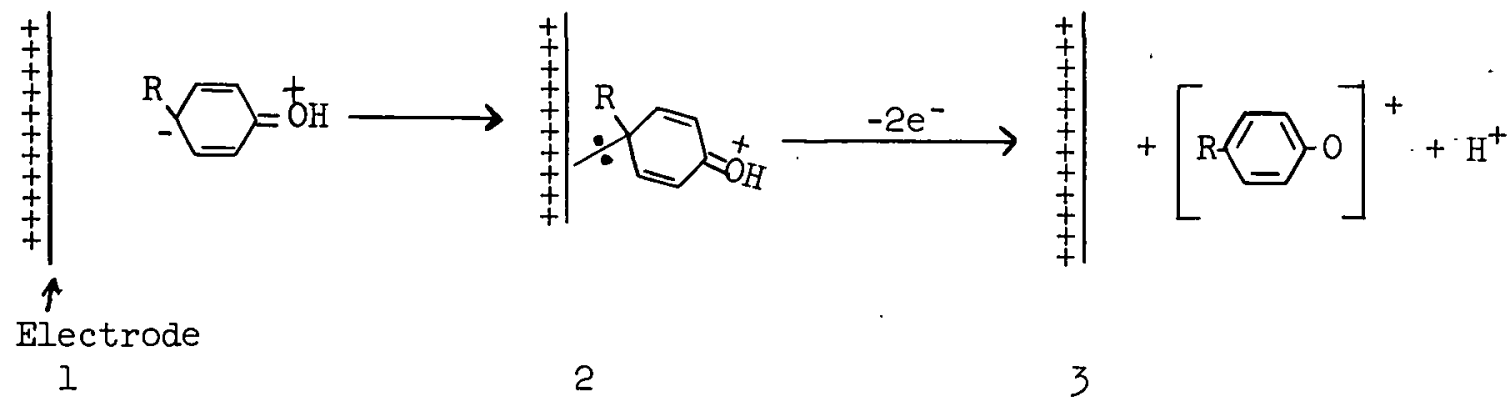

Figure 18. Schematic Representation for the Electrode Reaction of a Phenol

This concludes the present discussion of the electrode reactions of nonionized phenols in acetonitrile. A further discussion concerning the voltammetry of 2,6-dimethoxyphenol, isoeugenol, and the effect of pyridine on the voltammetry of phenols in acetonitrile is contained in the Appendix. 
FREE RADICAL MECHANISM OF PHENOXIDE ANIONS IIN ACETONITRILE

The phenoxide anions were obtained by adding tetraethylammonium hydroxide (10\% aqueous solution) in an amount approximately equivalent to the concentration of the phenolic hydroxyl. Excesses of hydroxide ion were avoided since this will cause hydrolysis of the acetonitrile. The phenoxide anions in acetonitrile are sensitive to atmospheric oxidation. The rate of oxidation depends to some extent on the phenol. Vanillin is relatively stable, while 2,6-di-tert-butylphenol gives a pale green coloration in a half hour which deepens to a dark blue-green in twelve hours. In the latter case 2,6,2',6'-tetra-tert-butyldiphenoquinone was formed.

\section{HINDERED PHENOIS}

In an initial series of experiments with 2,6-di-tert-butylphenol a stock solution of the phenoxide anion was prepared, and this was appropriately diluted in the voltammetric cell. The results are shown in Table VII, Experiments No. I through No. 4. The values for $-\underline{i} \underline{D} \underline{C}$ in these experiments were significantly lower than would be expected for a one-electron transfer because of the atmospheric oxidation of the phenol. In Experiment No. 5 of the table the tetraethylammonium hydroxide was added directly to the deaerated solution of the phenol in the voltammetric cell just before the curve was recorded. Here the recorded value of ${ }_{\underline{1}} / \underline{C}$ is $-21.1 \mu \mathrm{a}: / \underline{\mathrm{mM}}$ which is approximately the expected result for a one-electron transfer on electrode No. 8.*

Curve No. 6 of Table VII was recorded after the solution used for Curve No. 5 had stood in the cell for one hour under a nitrogen atmosphere. A significant

\footnotetext{
*The diffusion current constants of the nonionized phenols on this electrode ranged from -35 to $-50 \mu \mathrm{a} . / \mathrm{mM}$ in Table VI.
} 
decrease in $-\underline{i} \underline{D} / \underline{C}$ is noted. This is not surprising since the phenoxide anion itself is a strong base in acetonitrile and might be expected to promote hydrolysis reactions of the solvent.

\section{TABLE VII}

VOLTAMMETRY OF THE ANION OF 2,6-DI-TERT-BUTYLPHENOL IN ACETONITRILE

(Electrode No. 8, supporting electrolyte 0.1M tetraethylammonium perchlorate)

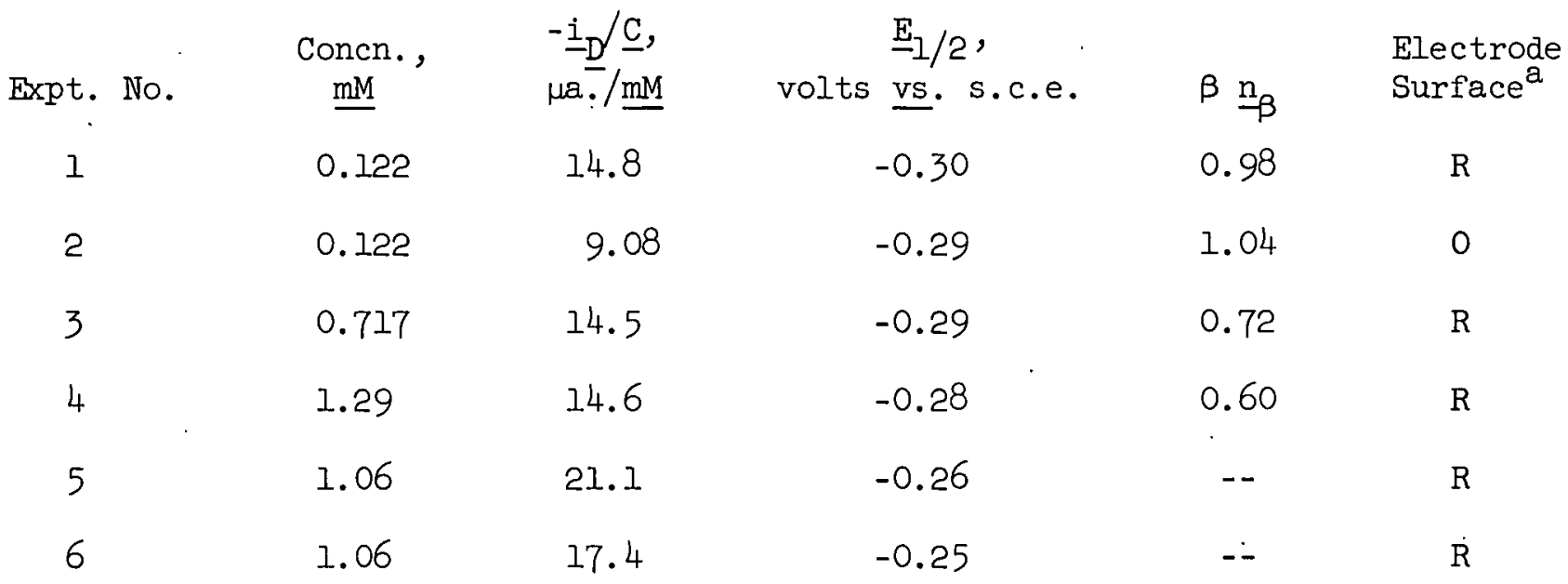

\footnotetext{
$a_{R}=$ reduced platinum surface.
}

$0=$ oxidized platinum surface.

The effect of an oxide film on the electrode surface in the reaction of the phenoxide anion is shown in Experiments 1 and 2 of Table VII. There is a 39\% reduction of the diffusion current but no effect on the half-wave potential or apparent reversibility from logarithmic analysis. This is probably a general effect for the phenoxide anions, as similar behavior has been noted in basic aqueous solutions with the vanillinate anion (see page 106). The decrease of the numerical value of $\underline{i}_{\underline{D}} / \underline{C}$ on an oxidized electrode suggests that specific active adsorption sites on the platinum surface are necessary for the reaction of the phenoxide anion to occur. 
Since the effect of an oxide film is totally different for the electrode reaction of the phenoxide anion than for the electrode reaction of the nonionized phenol*, a different electrode mechanism is indicated.

It will be noted in Table VII that the value of $\beta n_{-\beta}$ approaches 1.0 as expected for a reversible reaction at a low level of concentration, but decreases at higher levels of concentration. This behavior might be the result of rapid dimerization and reoxidation reactions of the phenoxy free radical formed as the product of the initial electrode reaction. The trend toward a limiting value of 1.0 at the lower level of concentration indicates that the primary electrode process is probably. a reversible reaction.

The anions of 2,6-DTBC and 2,4,6-tri-tert-butylphenol were not studied in detail because abnormally low current levels were observed for these phenoxides without the addition of excess tetraethylammonium hydroxide. For the latter phenoxide the half-wave potential was approximately -0.36 volt in the concentration range from .05 to $0.1 \mathrm{mM}$.

\section{VANILLINATE ANION}

\section{VOLTAMMETRY}

The phenoxide anion of vanillin was selected for more detailed study because it is relatively stable in acetonitrile solution, and because it gave little evidence of film formation at lower levels of concentration. It was found, however, that at higher levels of concentration (ca. ImM) that it was not possible to perfectly retrace a voltammogram without cleaning the electrode in hot chromic

* See p. 43 and p. 71 for a discussion of the effect of an oxide film in the reaction of the nonionized phenol. 
acid although severe filming as evidenced by peaked waves did not occur. All of the voltammetric results in this section were obtained on a reduced electrode.

A series of voltammograms with a solution $1.09 \mathrm{mM}$ in vanillin, and with successively increasing concentrations of tetraethylammonium hydroxide are shown in Fig. 19. It will be noted that before addition of base the vanillin displays a wave at 1.43 volts with $\underline{i}_{\underline{D}} / \underline{C}=-13.2 \mu \mathrm{a} . / \mathrm{mM}$ on electrode $\mathrm{V}-3$. This represents the two-electron transfer in unbuffered acetonitrile which was discussed for other phenols in the previous section. From the experiments with 2,6-DTBC on this electrode the value of $\underline{i}_{\underline{D}} / \underline{C}$ for a two-electron transfer should be approximately -9.6 $\mu \mathrm{a} . / \mathrm{mM}$, so in the case of vanillin a net $\underline{\mathrm{n}}$ value of about 2.7 is indicated.

With the addition of tetraethylammonium hydroxide to the solution of vanillin a new wave representing the vanillinate anion occurs at 0.25 volt. This wave reaches a limiting value for $\underline{\underline{i}}_{\underline{D}} / \underline{C}$ of $-4.45 \mu \mathrm{a} . / \underline{\mathrm{mM}}$ with the addition of 1.2 to. 2.5 equivalents of hydrogen ion, thus indicating a one-electron process. At the same time, a second, smaller wave, has occurred at ca. to.56 to 0.59 volt. The first wave represents the primary electrode process, which is believed to be the removal of one electron from the phenoxide anion to give a phenoxy free radical. The origin of the second wave is uncertain.

With the addition of further excesses of tetraethylammonium hydroxide to 3.7 and 5.0 times the equivalent weight of vanillin present an entirely different reaction is evidenced in Fig. 19. The half-wave potential for this process is 0.4 to 0.47 volt which is considerably more positive than the reaction for the vanillinate anion at 0.25 volt. A possible explanation for this shift is that the excess hydroxyl ion combines with the aldehyde group of vanillin to give the adduct,. XXII, according to Equation (20), and that XXII reacts according to Equation (2l) in a 


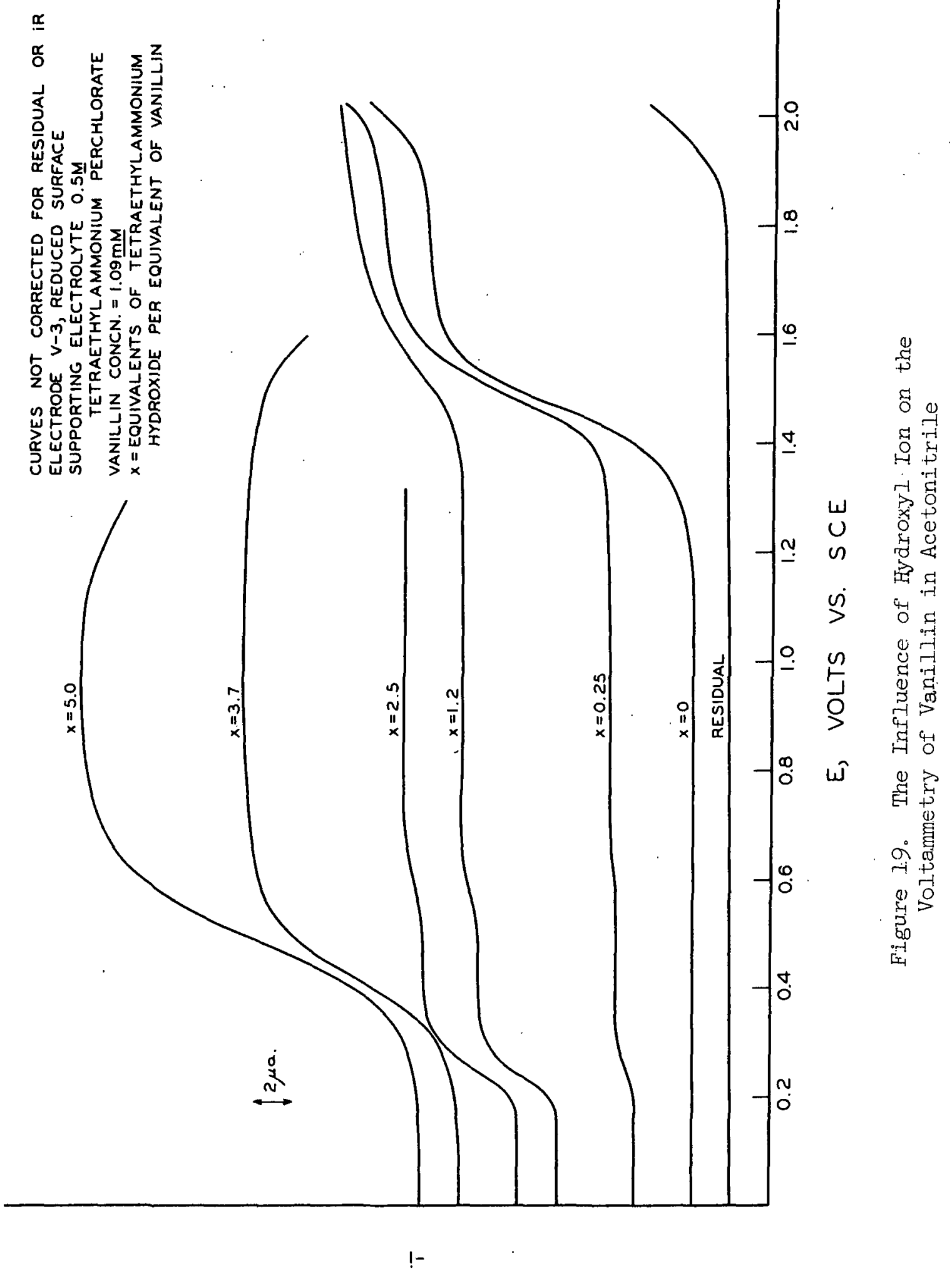


two-electron reaction to give vanillic acid. The adduct, XXII, is believed to be the intermediate in the Cannizzaro reaction (2I), and it has been suggested by Pearl (71) that a similar mechanism is responsible for the high yields of vanillic acid obtained from vanillin by silver oxide oxidation in the presence of excess sodium hydroxide. It is quite logical that XXII would form readily in acetonitrile solution since the solvent itself has very little ability to solvate hydroxyl ions. The height of the waves in Fig. 19 would indicate that considerably more than a two-electron reaction occurred when excesses of hydroxide ion were present in the solution. It is, however, quite certain from the correlation on page 95, and from the brief studies with 3,5-di-tert-butyl-4-hydroxybenzoic acid (page 70), that the vanillinate anion would react at a lower potential than that of the primary electrode process. Very probably a decarboxylation would occur as was postulated for the formation of the quinone in the electrochemical oxidation of 2,6-DTBC. Thus, it is not surprising that the apparent $\underline{\mathrm{n}}$ value is greater than two.

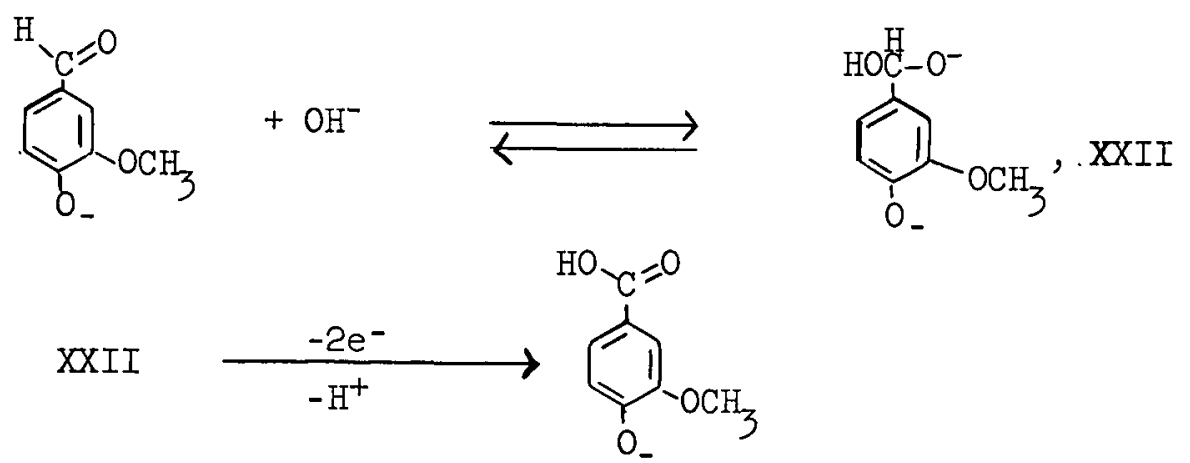

The reaction of vanillin in the presence of excesses of base was not further investigated because it did not represent a reaction of the phenoxide group. It would, however, be of considerable interest as an extension of the present study to observe the electrochemical behavior of other aldehydes in the presence of excess hydroxyl ion. 
The two waves of the vanillinate anion (no excess hydroxyl ion) are given for concentrations from 0.05 to $0.6 \mathrm{mM}$ in Table VIII. The first wave at these concentrations represents approximately a one-electron transfer because the values of ${ }_{\underline{i}} \mathrm{D} / \underline{\mathrm{C}}$ range from -19.5 to $-22.6 \mu \mathrm{a} . / \underline{\mathrm{mM}}$ on electrode No. 8 . * The second wave becomes smaller with increasing concentration. Thus, the ratio of $\underline{i}_{\underline{D}} / \underline{C}$ for the second wave to $\underline{i}_{\underline{D}} / \underline{C}$ for the first wave decreases from 0.64 to 0.31 in the concentration range of Table VIII. With further increases in concentration the height of the second wave continues to decrease. From Fig. 19 the ratio of wave heights is 0.24 at a concentration of $1.09 \mathrm{mM}$, and from Fig. 21 the second wave is completely absent at a concentration of approximately $8 \mathrm{mM}$.

\section{TABLE VIII}

\section{VOLTAMMETRY OF THE VANILLINATE ANION}

(Electrode No. 8, reduced surface; supporting electrolyte $=0.1 \mathrm{M}$ tetraethylammonium perchlorate)

\begin{tabular}{|c|c|c|c|c|c|c|c|}
\hline \multirow[b]{2}{*}{$\begin{array}{l}\text { Conen., } \\
\mathrm{mM}\end{array}$} & \multicolumn{3}{|c|}{ Wave I } & \multicolumn{3}{|c|}{ Wave II } & \multirow[b]{2}{*}{ Ratio ${ }^{2}$} \\
\hline & $\begin{array}{l}-\underline{\underline{i}} / \mathrm{c} \\
\mu \mathrm{a}\end{array}$ & $\begin{array}{c}\underline{E}_{1 / 2}, \\
\text { volts } \underline{\text { vs. s.c.e. }}\end{array}$ & $\beta \underline{n}_{\beta}$ & $\begin{array}{l}-\underline{\underline{i}} / \mathrm{d} \text {, } \\
\mu \mathrm{a} \cdot / \mathrm{mM}\end{array}$ & $\begin{array}{c}E_{1 / 2}, \\
\text { volts } \underline{\text { vs. s.c.e. }}\end{array}$ & $\beta \underline{n}_{\beta}$ & \\
\hline 0.0530 & 20.0 & 0.22 & 1.01 & 12.8 & 0.49 & 0.55 & 0.64 \\
\hline 0.106 & 19.5 & 0.22 & 1.05 & 12.4 & 0.53 & 0.47 & 0.64 \\
\hline 0.368 & 19.7 & 0.25 & 1.02 & 8.1 & 0.53 & 0.51 & 0.41 \\
\hline 0.619 & 22.6 & 0.22 & 1.02 & 7.2 & 0.56 & 0.61 & 0.31 \\
\hline
\end{tabular}

\footnotetext{
* Compare with the two-electron reactions of Table VI.
} 
The first wave of the vanillinate anion may be explained as the reversible ( $\beta \underline{n}_{\beta}=1$ ) removal of one electron to give the phenoxy free radical by Equation (22). One of the reaction products from this free radical, shown by the electrolyses which are discussed in a later section is dehydrodivanillin. This could form from the coupling* of two free radicals by Equation (23).
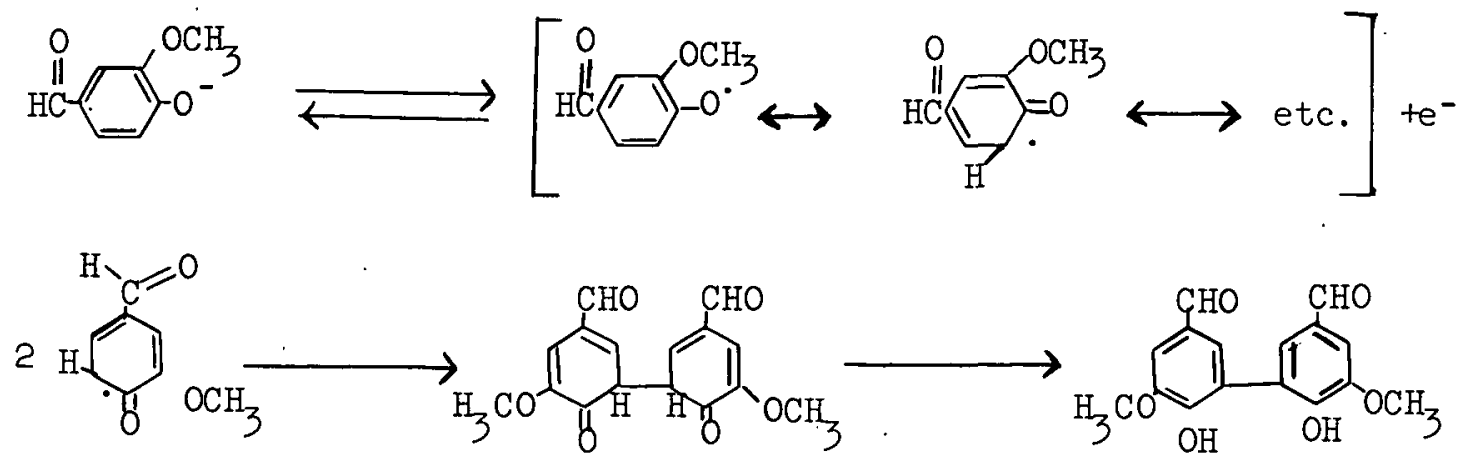

Since dehydrodivanillin was known to be a product of the electrode reaction, its voltammetric behavior was investigated briefly in the presence of vanillin. The results are given in Fig. 20. Curve 1 of the figure represents the diphenoxide of dehydrodivanillin,.XXIII, at a concentration level of $0.748 \mathrm{mM}$. The first wave for this species occurs at approximately -0.01 volt with $\underline{i}_{\underline{D}} / \underline{C}=-4.6 \mu \mathrm{a} . / \mathrm{mM}$ which would suggest the removal of one electron to form the radical anion, XXIV. A second deformed wave occurs at approximately t0.32 volt which could represent the removal of a second electron from the phenolic hydroxyl of XXIV.

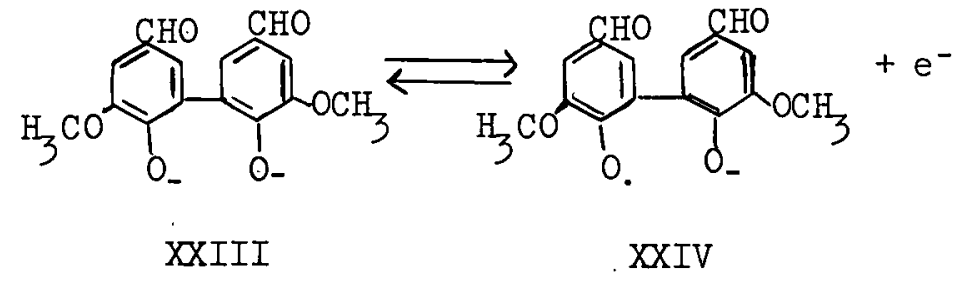

\footnotetext{
*It is not certain that the dimer forms by coupling of two free radicals. Authorities on the oxidative dimerization of phenols seem to differ in opinion as to whether the coupling involves two free radicals or a free radical and $a$. monomer $(\underline{72}-14)$. The present author favors the former mechanism.
} 


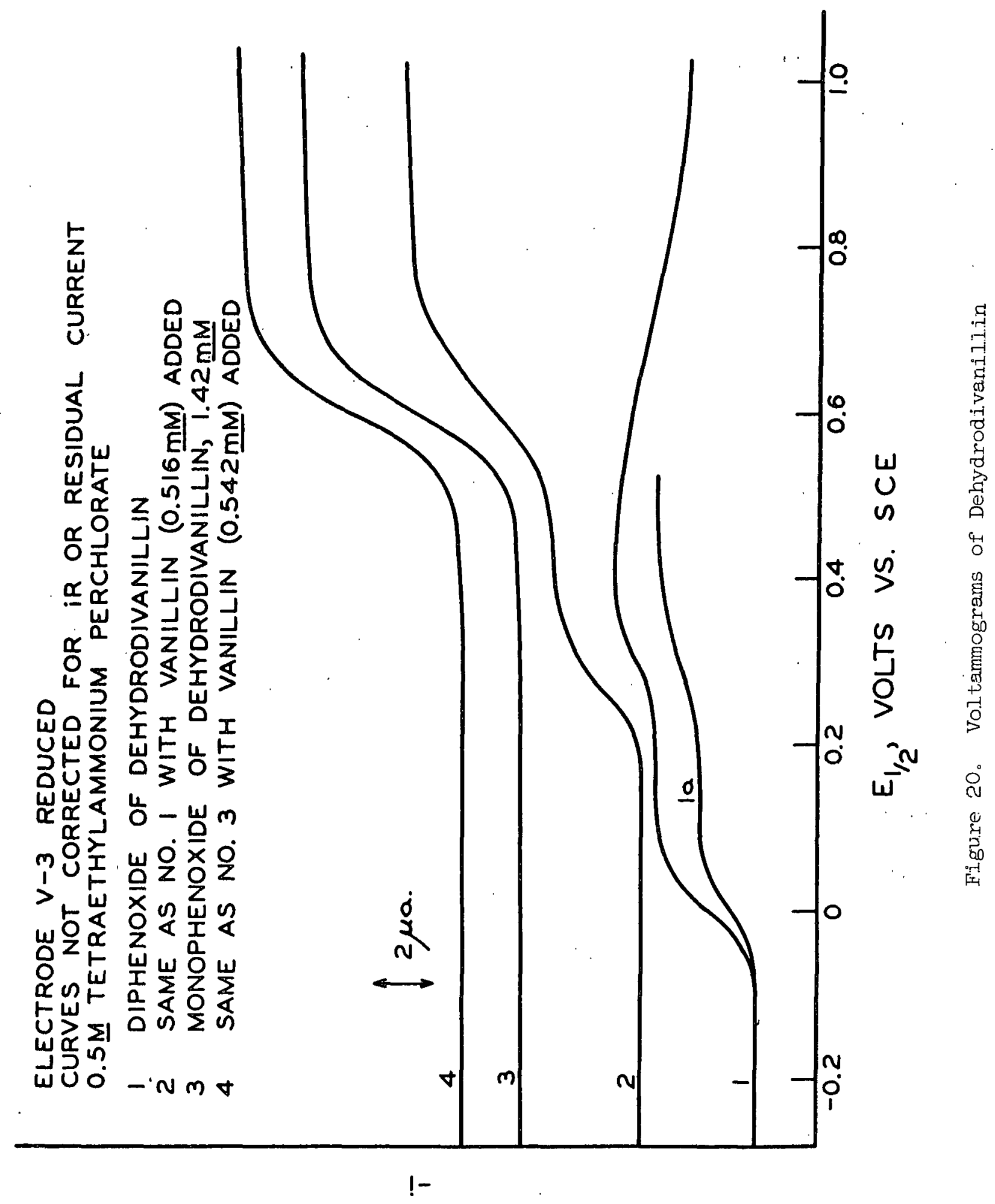


After the solution had remained in the cell for 1.5 hours the wave height was reduced as shown in Curve No. la. The addition of vanillin at a concentration level of $0.516 \mathrm{mM}$ to this solution produced the result shown in Curve No. 2. Here the waves of the diphenoxide anion of dehydrodivanillin are completely absent, but the wave for the vanillinate anion at +0.25 volt is present, and a new wave caused by the monophenoxide, XXV, of the dehydrodivanillin is present at 0.59 volt. This behavior indicates that in acetonitrile the ionization constant of vanillin is sufficiently greater than the second ionization constant of dehydrodivanillin so that the equilibrium of Reaction (25) lies far to the right.<smiles>COc1cc(C=O)cc(-c2cc(C=O)cc(OC)c2OC)c1</smiles>

XXIII<smiles>O=Cc1ccc(O)c(O)c1</smiles>

$$
\text { (1) }
$$$$
\text { 政 }
$$

The wave caused by $\mathrm{XXV}$ is shown without added vanillinate anion in Curve No. 3. The value of $\underline{i}_{\underline{D}} / \underline{C}$ for this wave is $-5.07 \mathrm{\mu a} . / \underline{m M}$ indicating a one-electron reaction to give the free radical, XXVI, by Reaction (26). When vanillin (0.542mM) was added to this solution (Curve No. 4) there was no significant change in the voltammogram. This indicates that the vanillin was not ionized--hence the equilibrium of Reaction (27) must lie far to the right in acetonitrile.<smiles>COc1cc(C=O)cc(-c2cc(C=O)cc(OC)c2-c2cc(C=O)cc(OC)c2O)c1O</smiles><smiles>COc1ccc(C=O)cc1-c1cc(C=O)cc(OC)c1-c1cc(C=O)cc(OC)c1O</smiles> 
Since the second wave of the vanillinate anion occurred at about the same potential as the wave of $\mathrm{XXV}$, and since $\mathrm{XXV}$ would be expected in the solution from Reactions (23) and (27), it would be reasonable to think that the origin of the second wave of the vanillinate anion was Reaction (26). However, five factors argue against this hypothesis.

1. There is a significant difference between $E_{1 / 2}$ for the second wave of the vanillinate anion at a low level of concentration and $E_{1 / 2}$ for Reaction (26), to.50 volt as compared to to. 59 volt.

2. The current ratio of Wave II to Wave I for the vanillinate anion is highest at the lower concentration levels $(<0.1 \mathrm{mM})$. Since one would expect Reactions (23), (27), and (26) to go more readily at high concentration levels, this is the reverse of the predicted trend.

3. The maximum current ratio of Wave I to Wave II predicted from Equations (23), (27), and (26) is 0.5. Therefore, the observed value of 0.6 at concentrations less than $0.1 \mathrm{mM}$ is too large.

4. For the monophenoxide of dehydrodivanillin, $\beta \underline{n}_{\beta}$ was calculated as 1.47 . Yet the values for the second wave of the vanillinate anion range from 0.47 to 0.51 .

5. The experiments in aqueous media indicated that the second wave of vanillin is $\mathrm{pH}$ independent. This would not be the case with Reaction (26).

Thus, the origin of the second wave of vanillin remains unknown. The case is complicated because the reaction sequence represented by Equations (23), (27), and (26) will lead to a decrease of the apparent $\underline{n}$ value for the first wave to 0.66 because the vanillinate anion is used up by. (27) as well as by the primary electrode process. It wili in fact be noted that in Fig. 19 a current level corresponding to a one-electron process was not reached until an excess of hydroxyl ion was added to the system. In the $8 \mathrm{mM}$ solution used for the electrolysis, Fig. 2l, the current 





level was much lower than would be predicted from the loss of the vanillinate anion by-Equation (27). This may have been caused by the insolubility of dehydrodivanillin in a nonionized form, and by side reactions leading to polymeric products.

\section{ELECTROLYSIS}

Initial electrolyses in the millicoulometric cell revealed dehydrodivanillin as the main reaction product. From the voltammetric studies it was known that the reaction could only be carried to $66.7 \%$ completion with addition of enough hydroxyl ion to neutralize the original vanillin in the solution because the vanillinate anion would be used up by Reaction (27) as well as by the electrode reaction. An excess of hydroxyl ion, on the other hand, 'could not be tolerated because of the reaction of vanillin in the presence of excess hydroxyl (Fig. 19), and because of the reaction of the diphenoxide ion of dehydrodivanillin.

It was reasoned that these difficulties could be overcome by adding enough hydroxyl at the beginning of the electrolysis to neutralize the vanillin, and periodically adding additional hydroxide ion during the electrolysis at a rate of 0.5 equivalent per Faraday of electricity. Voltamograms run at intervals during the electrolysis are given in Fig. 2l. Curve No. 2 at the beginning of the electrolysis shows an approximate diffusion current constant of only $-1 \mu \mathrm{a} / \mathrm{mM}$, a considerable decrease from the value at lower concentration levels. The reasons for this decrease are discussed in the previous section. The second wave of the vanillinate anion is not apparent under these conditions.

The other curves were obtained at intervals through the electrolysis as discussed in the experimental section. It will be noted that a second wave corresponding to the monophenoxide of dehydrodivanillin is apparent in these curves, and becomes larger at longer periods of electrolysis. Apparently, this wave is not inhibited at high concentration levels as is the wave for the vanillinate anion. 
The electrolysis was stopped after 956 minutes when, as will be noted in Fig. 21 , the addition of excess base beyond the calculated total requirement of 1.5 equivalents per equivalent of vanillin did not produce a distinct wave for the vanillinate anion. From the current level for the diphenoxide of dehydrodivanillin in Curve No. 6, the yield of dehydrodivanillin should have been approximately $62 \%$. The recovered crude yield of dehydrodivanillin was $65.4 \%$ in close agreement with this figure.

The calculated coulometric $\underline{\mathrm{n}}$ value for the reaction based on the recovered dehydrodivanillin was 1.77 . Since the true $\underline{\mathrm{n}}$ value for the formation of this product should be 1.0, there must have been some side reactions which consumed additional electricity. A logical course for such a side reaction in view of the results from the electrolysis of 2,6 -DTBC would be oxidation of vanillin to vanillic acid, and decarboxylation of the acid to form, eventually, quinoidal structures. Such a reaction would represent a net oxidation of six electrons. A rough calculation shows that only $8 \%$ of the original vanillin would have to be oxidized by such reactions to give an increase in the apparent coulometric $\underline{n}$ value from 1.00 to 1.77 . In addition to this side reaction, some polymerization processes may have occurred.

It is believed that an improved yield of dehydrodivanillin could be achieved in this electrolysis, because by paper chromatography the presence of unreacted vanillin in a fairly large amount was noted in the ether soluble fraction of the crude product of electrolysis.

The obtaining of dehydrodivanillin as the major product of electrolysis verifies the one-electron nature of the electrode reaction as postulated from the voltammetric studies. 


\section{CORRELATION OF HALF-WAVE POTENTIALS}

Equation (28) which is developed in Appendix A predicts the variation of $\mathrm{E}_{-1 / 2}$ with the Harmett $\sigma$ function for a reversible reaction at $25^{\circ} \mathrm{C}$. A plot for the phenoxides is given in Fig. 22. In this plot, $\sigma^{c}$ rather than $\sigma$ fitted the observed data for the para aldehyde substituent in vanillin. This is an indication that the electrode reaction in this case involves an attack on the oxygen atom of the ionized phenol.

$$
E_{1 / 2}-E_{1 / 2}^{0}=-0.0591 \sigma \rho
$$

The point for the doubly ionized form of 3,5-di-tert-butyl-4-hydroxybenzoic acid does not lie on the same line with the other three points. It is believed that the reason for this is that a $\sigma$ rather than a $\sigma^{c}$ value was used for the para carboxylate, because no literature value for $\sigma^{c}$ was available (2ll). Yet, since this group is directly conjugated with the phenolic oxygen, one would expect a $\sigma^{c}$ value to apply.

The value of $\rho$ calculated from the slope of the line in Fig. 22 is -6.63 . This is quite close to a $\rho$ value of -7.35 which was calculated from the data in $50 \%$ aqueous isopropanol at pH 5.6 of Suatoni, et al. (20) using Equation (34), and assuming that $\rho^{\prime}$ for $50 \%$ aqueous isopropanol is the same as $\rho^{\prime}$ for $50 \%$ aqueous ethanol; i.e., $\rho^{\prime}=2.55$ (70).

Although very few data points were available for the correlation of Fig. 22, completely different types of compounds are represented. Because of this and the excellent correlation which is evidently possible in aqueous media ${ }^{2}$, it is believed that the present correlation is quite general.

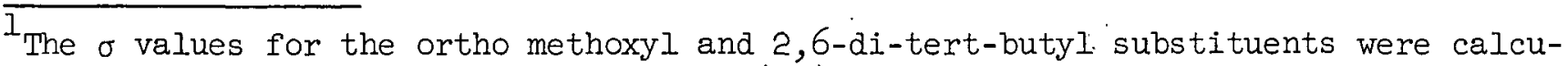
lated from the data of Suatoni, et al. (20) in the manner described on page 100. ${ }^{2}$ See discussion of aqueous buffered systems on page 99.
} 


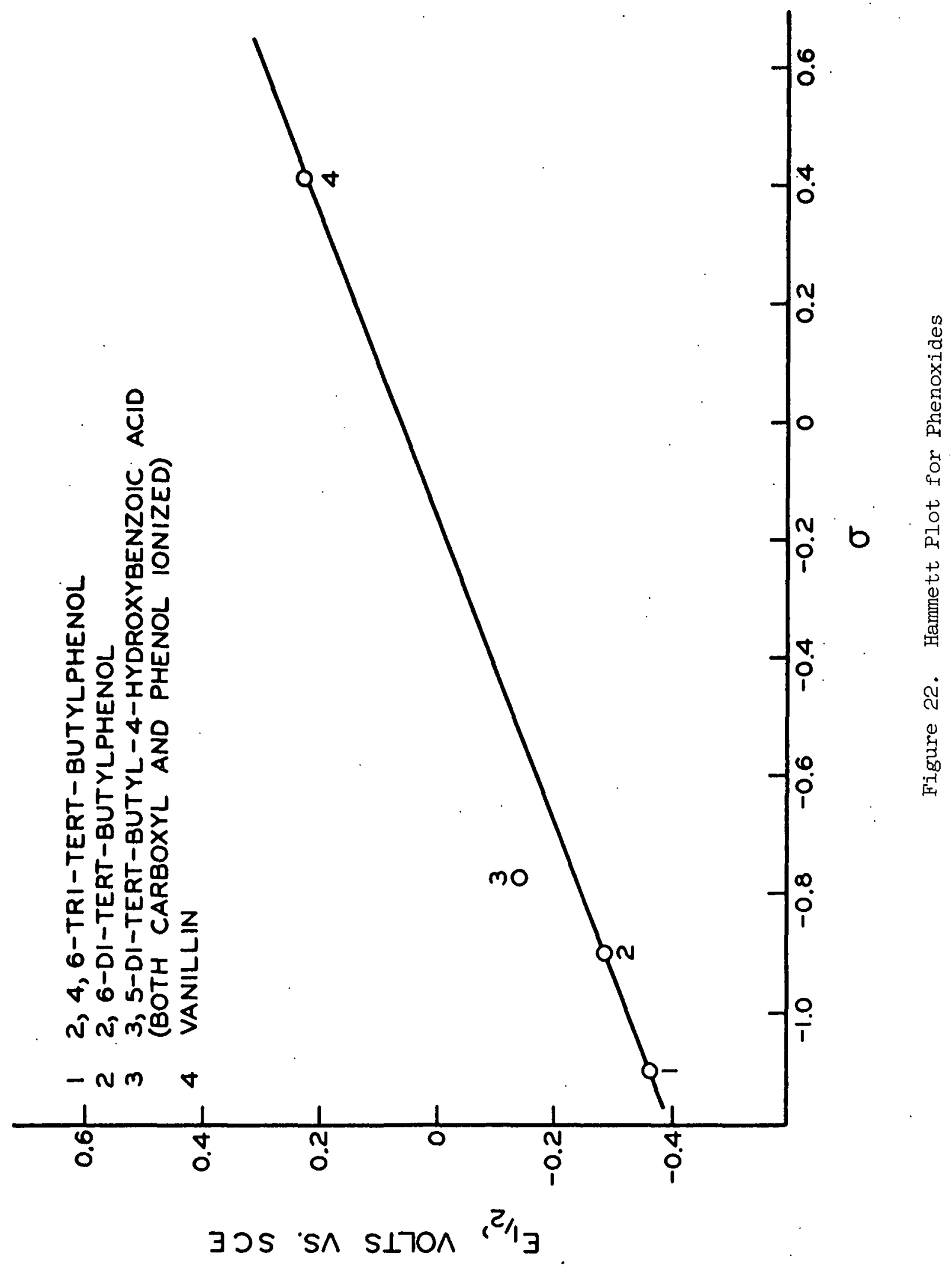


MECHANISM OF THE ELECTRODE. REACTION

The results obtained with the vanillinate anion in particular, and with the other phenoxides which were investigated have indicated the following characteristics for the primary electrode reaction:

1. One electron is transferred.

2. The reaction is voltammetrically reversible.

3. Diffusion currents on an oxidized platinum surface are much lower than on a reduced platinum surface.

4. In a Hammett-type correlation of half-wave potentials for a limited number of compounds $\sigma^{c}$ values rather than $\sigma$ values fit the observed data.

It has already been pointed out that the fourth characteristic indicates that the electron is removed from the phenolic oxygen. The third characteristic may also be interpreted as indicating the same thing. The reduction in current level on an oxidized surface must mean that specific active sites on the platinum surface are necessary for the reaction of the phenoxide. Since the small oxidation wave for hydroxyl ion is absent on an oxidized surface (르), it is reasonable to assume that the same surface sites are required for the oxidation of the phenoxide anions as for hydroxyl ions. The initial oxidation of hydroxyl ions evidently involves the removal of an electron from the oxygen atom and with the adsorption of the resulting free radical on the platinum surface (54)* according to Equation (29). When the platinum surface is covered with an oxide film Reaction (29) does not occur. Since the phenoxide anions also contain an oxygen atom with a localized negative charge the electrode reaction might well be expected to be similar to that of the hydroxyl anion. Carrying this analogy one step further, one wonders whether

\footnotetext{
* In the discussion of Laitenen and Enke only the reaction in acidic media is considered; hence, his equations discuss the formation of the intermediate Pt...OH from water rather than hydroxyl ion. However, in basic media the hydroxyl ion is believed to be the reacting species (60).
} 
the resulting phenoxy free radical is temporarily adsorbed on the platinum surface. This would seem probable, although the results of the present study do not permit any definite conclusion in this respect. It may be reasoned that, because of the resonance possibilities in the phenoxy free radical, a weaker adsorption would result than in the case of the hydroxyl free radical.

$$
\mathrm{Pt}+\mathrm{OH}^{-} \rightleftharpoons \mathrm{Pt} . \mathrm{OH}+\mathrm{e}^{-}
$$

A schematic representation of the proposed electrode reaction is given in Fig. 23. As the phenoxide anion approaches the electrode it becomes preferentially polarized so that the negative charge is located on the phenolic oxygen atom. A temporary bond with the positive electrode surface is formed and broken with the removal of one electron. The resulting phenoxy radical is stabilized through resonant structures as it leaves the electrode surface.

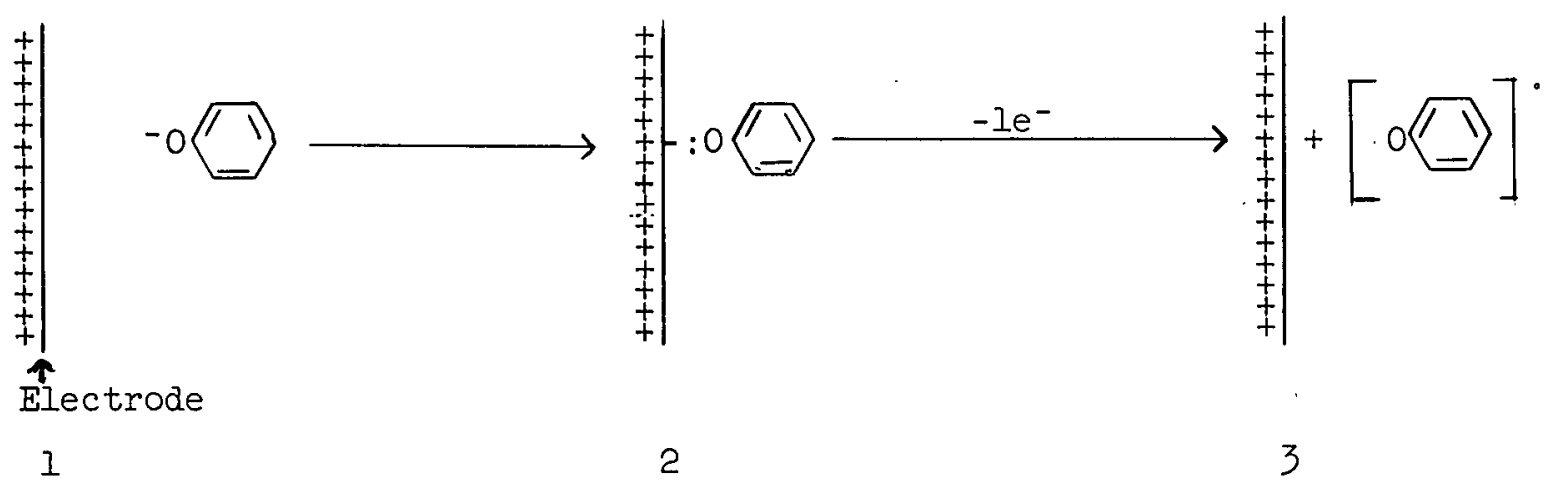

Figure 23. Proposed Reaction Mechanism of Phenoxide Anions 


\section{AQUEOUS BUFFERED SOLUTIONS}

From the previous results in acetonitrile it may be expected that the voltammetric behavior of phenols in aqueous solutions will vary depending on the $\mathrm{pH}$ of the media. Three distinct cases may occur.

1. At a sufficiently low level of $\mathrm{pH}$ the phenol is not appreciably ionized and an electrophilic attack on the aromatic nucleus should occur to give a mesomeric phenoxonium ion, providing that the supporting electrolyte solvent system has a sufficient anodic range for this reaction to occur. In this case the voltammetric characteristics should be similar to those observed for nonionized phenols in acetonitrile--(a) an irreversible two-electron, $\mathrm{pH}$ independent wave; (b) higher potentials and a lower apparent reversibility on an oxidized than on a reduced platinum electrode; and (c) a correlation of half-wave potentials for various para and meta substituents by the Hammett relationship using $\sigma$ rather than $\sigma^{c}$ values for aldehyde or carboxyl groups in the para position to the phenolic hydroxyl.

2. When the $\mathrm{pH}$ is sufficiently high the phenol is totally ionized, and a reaction to give a mesomeric free radical may be expected. Voltammetric characteristics should in this case be the same as were observed for the phenoxide anions in acetonitrile--(a) a reversible, pH independent, one-electron wave; (b) a lower diffusion current on an oxidized than on a reduced platinum electrode; (c) a correlation of half-wave potentials using $\sigma^{c}$ for carbonyl or carboxyl groups in the para position.

3. At intermediate ranges of $\mathrm{pH}$ both the ionized and the nonionized forms of the phenol are present. If as proposed by Hedenberg and Freiser (ㅁ), the rate of ionization of the phenol is very fast in comparison to the rate of the electrode reaction, the phenoxide anion should be preferentially oxidized. Except for $\mathrm{pH}$ dependence the observed voltammetric wave should have the same characteristics as 
the totally ionized phenol. The variation of half-wave potential with $\mathrm{pH}$ is given by Equation (30) at $25^{\circ} \mathrm{C}$.

$$
E_{I / 2}=E_{0}+0.0591\left\{\log K_{1} / K_{2}+\log \left(\frac{K_{D}+\left[H^{+}\right]}{K_{D}}\right)\right\}
$$

where $K_{-1}$ and $K_{-2}$ are constants from the approximate treatment of the diffusion problem at the electrode, and $\underline{K}_{-}$is the dissociation constant of the phenol.

At a high level of $\mathrm{pH}$, where $\underline{K}_{\underline{D}}>\left[\mathrm{H}^{+}\right]$, this equation can be simplified to

$$
\cdot E_{l / 2}=E_{0}+0.0591\left(\log \cdot K_{1} / K_{2}\right)
$$

In terms of the Hammett relationship this becomes

$$
E_{1 / 2}-E_{1 / 2}^{0}=-0.0591 \sigma \rho
$$

which is the equation previously given for the phenoxide anion in acetonitrile.

At a level of $\mathrm{pH}$ where $\left[\mathrm{H}^{+}\right] \gg_{\underline{-}}^{\mathrm{K}}$, Equation (30) simplifies to

$$
E_{I / 2}=E_{0}+0.0591\left(\log K_{I} / K_{2}-p H+p K_{D}\right)
$$

Thus, as the $\mathrm{pH}$ becomes lower a plot of $\mathrm{E}_{1 / 2}$ vs. $\mathrm{pH}$ should become a straight line with a slope of 0.0591 . The Hammett relationship for this case as developed in the Appendix is

$$
E_{l / 2}-E_{l / 2}^{0}=-0.0591 \sigma\left(\rho+\rho^{\prime}\right)
$$

where $\rho^{\prime}$ is the Hammett reaction constant for the ionization of phenols in the media used for the voltammetric studies.

Suatoni, et al. (20) have tabulated half-wave potential data for a variety of ortho, para, and meta-monosubstituted phenols at pH 5.6 in $50 \%$ aqueous isopropanol. Their plot of $\underline{E}_{1 / 2}$ vs. $\sigma$ fits the observed data with a maximum deviation 
of 0.08 volt. More important, however, is their finding that $\Delta \underline{E}_{1 / 2}$ values* are additive so that predictions of $\underline{E}_{1 / 2}$ for disubstituted compounds at the same $\mathrm{pH}$ to a precision of 0.01 volt are possible. This indicates the validity of a Hammetttype equation. Thus, where discrepancies exist in the Hammett correlation it should be possible to calculate adjusted $\sigma$ values for voltammetric purposes from knowledge of $\Delta \underline{E}_{1 / 2}$ and the slope of the plot of $E_{1 / 2}$ vs. $\sigma$. This same procedure can be used for ortho substituents where no $\sigma$ values are available. In the case of the 2,6disubstituted phenols where the additivity of $\Delta \mathrm{E}_{-1 / 2}$. values does not hold, a special value for the 2,6-disubstitution can be calculated. For example, for the cases of 2,6-di-tert-butyl, and ortho methoxyl substitution $\sigma$ values can be calculated as follows :

Slope of plot of $E_{1 / 2}$ vs. $\sigma=0.0591\left(\rho-\rho^{\prime}\right)$ in. Equation (34) $=-0.284(\underline{20})$.

For 2,6-di-tert-butyl substitution, $\Delta \underline{E}_{1 / 2}=0.255$ v. (20)

$$
\therefore \sigma_{2,6-d i-t-B u}=-0.255 / 0.284=-0.898
$$

For ortho methoxyl substitution, $\Delta \underline{E}_{1 / 2}=0.177 \mathrm{v}$.

$$
\sigma_{\text {ortho-OMe }}=-0.177 / 0.284=-0.623 \text {. }
$$

These were the values used for the correlation of the phenoxide anions in acetonitrile, Fig. 22.

The calculated $\sigma$ values can then be used together with Equations (31), (32), (33), and (34) to predict $E_{1 / 2}$ values at any level of $\mathrm{pH}$. This correlation should be valid except at a low level of $\mathrm{pH}$ where the $\underline{E}_{-1 / 2}$ value predicted by Equation (33)

${ }^{*} \underline{E}_{-1 / 2}=\underline{E}_{1 / 2}$ for phenol - $E_{1 / 2}$ for substituted phenol. 
becomes so high that the two-electron electrophilic attack on the aromatic nucleus of the nonionized phenol occurs before the one-electron reaction of the ionized phenol. Reactions of other functional groups may also occur under special conditions--for instance, the direct attack of an aldehyde group in strong basic solution, as was evidently observed for vanillin in the presence of excess base in acetonitrile.

In order to test the hypotheses advanced in this section, some brief experiments were conducted in aqueous systems using two compounds, 2,6-DTBC, and vanillin. So that the results would be comparable with those of other authors $(\underline{13}, 1,4,17,20$ ) the solvent system used was a 50\% mixture of isopropanol and water. The buffer mixtures were prepared at a constant ionic strength (0.1M) as recommended by Elving ( $\underline{75})$.

$$
\text { 2,6-DI-TERT-BUTYL-p- }- \text { CRESOL }
$$

Voltammograms using 2,6-DTBC at a concentration level of $0.530 \mathrm{mM}$ are given in Fig. 24. The rotating carbon electrode was used in this work because of the extreme effects of oxide films on a platinum electrode which were noted with vanillin (vide infra). At $\mathrm{pH}$ levels of 1.4 and 3.7 a pH independent irreversible wave is observed which presumably is the electrophilic attack on the aromatic nucleus of the nonionized phenol. The half-wave potential for this reaction is to.98 volt vs. s.c.e. as compared to +1.23 volts at the same concentration level in the acetonitrile solvent. The direction of this shift is the same as was observed upon addition of $4 \%$ water to the acetonitrile but the magnitude as expected is somewhat greater. This shift represents primarily a change in the activation energy for the irreversible electrode reaction.*

\footnotetext{
*Changes in the liquid junction potential, and hydrodynamic and diffusivity variables are also included in the change of half-wave potential between different solvents, but these effects are generally of a lower order of magnitude than observed here.
} 


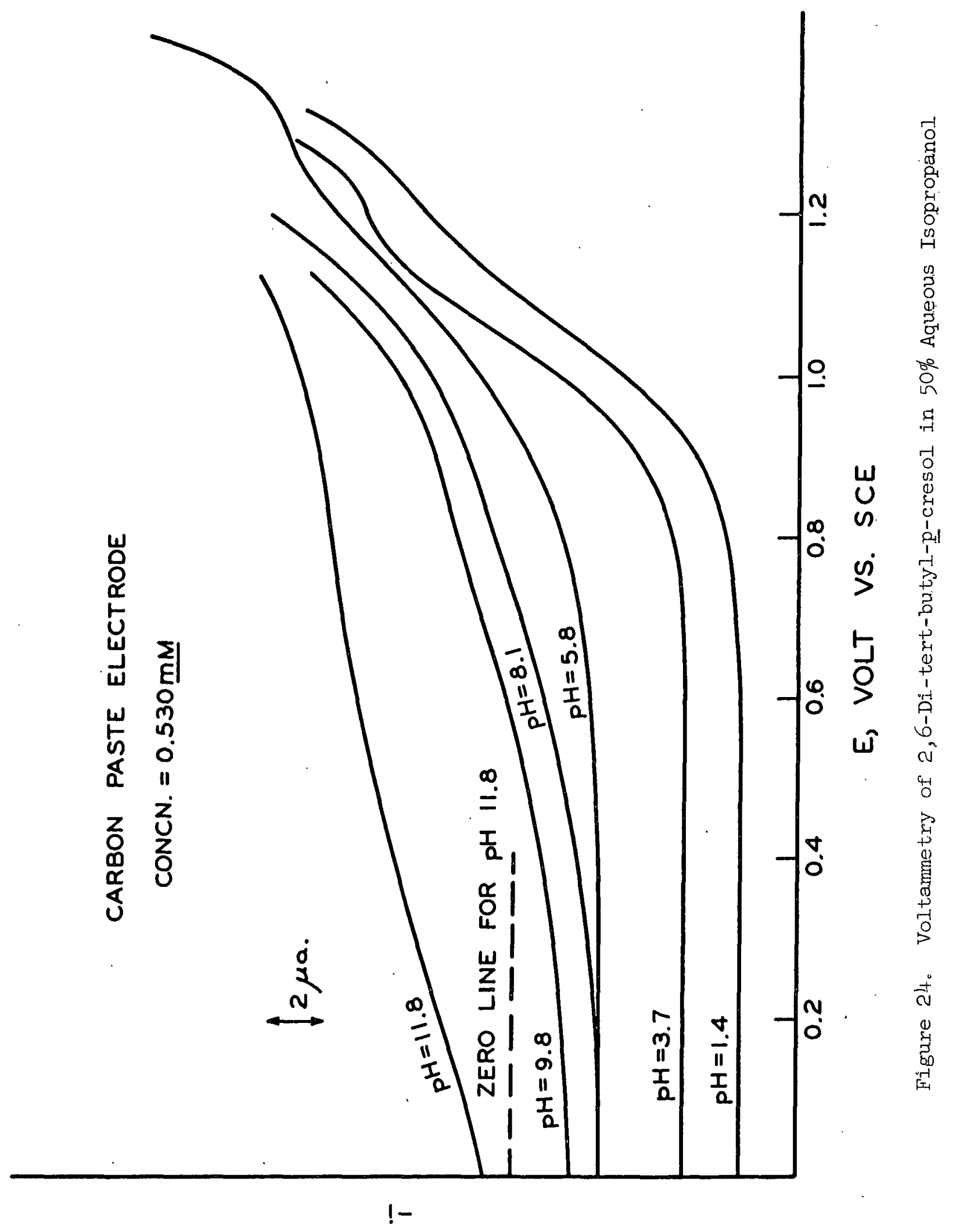


The diffusion current constant for the reaction at $\mathrm{pH} 1.4$ and 3.7 is about $-20 \mu \mathrm{a} . / \mathrm{mM}$. By comparison with the results in acetonitrile in the presence of $4 \%$ water one would expect that this should represent something greater than a twoelectron process $(2.58=$ average $\underline{n}$ value with $4 \%$ water in acetonitrile, page 57$)$. Yet the $\underline{i}_{-} / C$ value for vanillin on the carbon electrode in the isopropanol water system, an obvious one-electron reaction, was also $-20 \mu \mathrm{a} . / \mathrm{mM}$ (vide infra). On the basis of the figure for vanillin, the diffusion current constant for 2,6-DTBC should have been approximately $-52 \mu \mathrm{a}: / \mathrm{mM}$, assuming a net $\underline{\mathrm{n}}$ value of 2.6 . In other words, the observed $\underline{n}$ value is low by a factor of 2.6 .

A similar result for other hindered phenols has been reported by Nash, et al. (17) at $\mathrm{pH} 7.0$ where a one-electron reaction should occur. In this case, current levels for vanillin and phenol, respectively, were 2.4 , and 3.0 times as high as the current level of 2,6-di-tert-butylphenol. For 2,4,6-tri-tert-butylphenol these factors were even higher (3.1 and 3.8). Similarly, Suatoni, et al. (20) at pH 5.6 report the current level for phenol as 2.5 times the current level of 2,6di-tert-butylphenol.

The repeated reports of abnormally low current levels for the hindered phenols in the isopropanol water system must be attributed to a difference in diffusivity between these and other phenols. The change in diffusivity for this change in diffusion current can be approximated from the relationship $\underline{i}_{\underline{D}} / \underline{\mathrm{C}} \propto \underline{\mathrm{D}}^{0.66}(\underline{62}, \underline{63})$. Thus, a 2.6-fold change in the value of $i_{-\underline{D}} / \mathrm{C}$ requires a 4.3 -fold difference in diffusivities. This great a change is an order of magnitude greater than would be expected on the basis of molecular size or solvation effects*, and must therefore indicate that the hindered phenols are to some extent aggregated in $50 \%$ aqueous

*Note that in acetonitrile no very significant dependence of limiting currents on molecular size was ever observed. Refer to Tables V and VI. 
isopropanol. This result points out the need for diffusivity studies in conjunction with electrochemical investigations.

In Fig. 24 it will be noted that, as the $\mathrm{pH}$ is increased, a deformed wave at lower potentials occurs for 2,6-DTBC. This wave evidently represents the oneelectron reaction of the ionized phenol. Other workers. (18, 23) have also reported poorly formed waves for 2,6-DTBC on both platinum and carbon electrodes, particularly at lower $\mathrm{pH}$ levels, although they evidently did not observe the two-electron wave at $\mathrm{pH} 1.4$ and 3.7 .

A similar result in a single trial at $\mathrm{pH} 8.1$ was observed in the present study for 2,6-di-tert-butylphenol, yet neither Nash, et al. (17) nor Suatoni, et al. (20) report any particular difficulty in obtaining voltammetric curves for this compound. Indeed, the former author reports a $\beta n_{\beta}$ value of 1.09 which would indicate an essentially reversible wave. Since these authors prepolarized their wax-impregnated carbon electrodes at an anodic potential, the same method was tried in the present study with no significant improvement in results. It was noted, however, that if the carbon electrode was cathodically treated at a potential beyond the point of hydrogen discharge, a normal appearing wave could be obtained at pH 11.8 which was superimposed on the peak for adsorbed hydrogen.

The exact reason for the poorly formed waves with the hindered phenols in the present study is unknown. Since in acetonitrile a fully developed one-electron wave for 2,6-DTBC could not be observed without the addition of considerable excess base, there is a reason to suspect that this phenomenon is related to the ease of ionization. Specific adsorption effects of the phenoxy free radicals may also be important. 


\section{VANILUIN}

Voltammograms for vanillin at a concentration of $0.12 \mathrm{mM}$ were obtained on both a platinum and a carbon electrode. The curves for the carbon electrode are presented in Fig. 25. The following effects of platinum surface oxide were noted:

1. At a $\mathrm{pH}$ of 5.8 the current level was lower on an oxidized than on a reduced electrode, and the second wave of vanillin noted on the carbon electrode in Fig. 25 could not be obtained even on a reduced platinum surface.

2. On a reduced electrode at $\mathrm{pH}$ levels of 8.1 and higher the current level was lower than at $\mathrm{pH}$ levels of 5.8 and lower because the surface oxide formed before the wave for vanillin.

3. At a $\mathrm{pH}$ level of 11.8 no wave for vanillin could be obtained even on a reduced surface.

The half-wave potentials were essentially the same for both platinum and graphite electrodes indicating that the same electrode process was occurring.

In Fig. 25, it will be noted that the second small wave is clearly defined only at a pH level of 5.8. At a lower pH it is blended with the first wave, and at a higher $\mathrm{pH}$ it evidently occurs beyond the decomposition point of the solution. It, therefore, appears to be $\mathrm{pH}$ independent.

A plot of half-wave potential vs. $\mathrm{pH}$ for the first wave of vanillin is given in Fig. 26. The limiting slope at low $\mathrm{pH}$ is -0.0591 as predicted by Equation (33). The limiting potential at high $\mathrm{pH}$ is to.536 volt vs. s.c.e. Thus, from Equation (31)

$$
\underline{E}_{0}+0.0591 \log \left(\underline{K}_{-} / \underline{K}_{2}\right)=+0.536
$$

From Equation (33) the intercept at zero $\mathrm{pH}$ is 


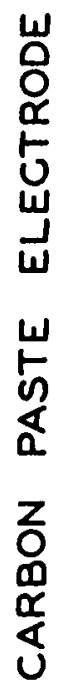

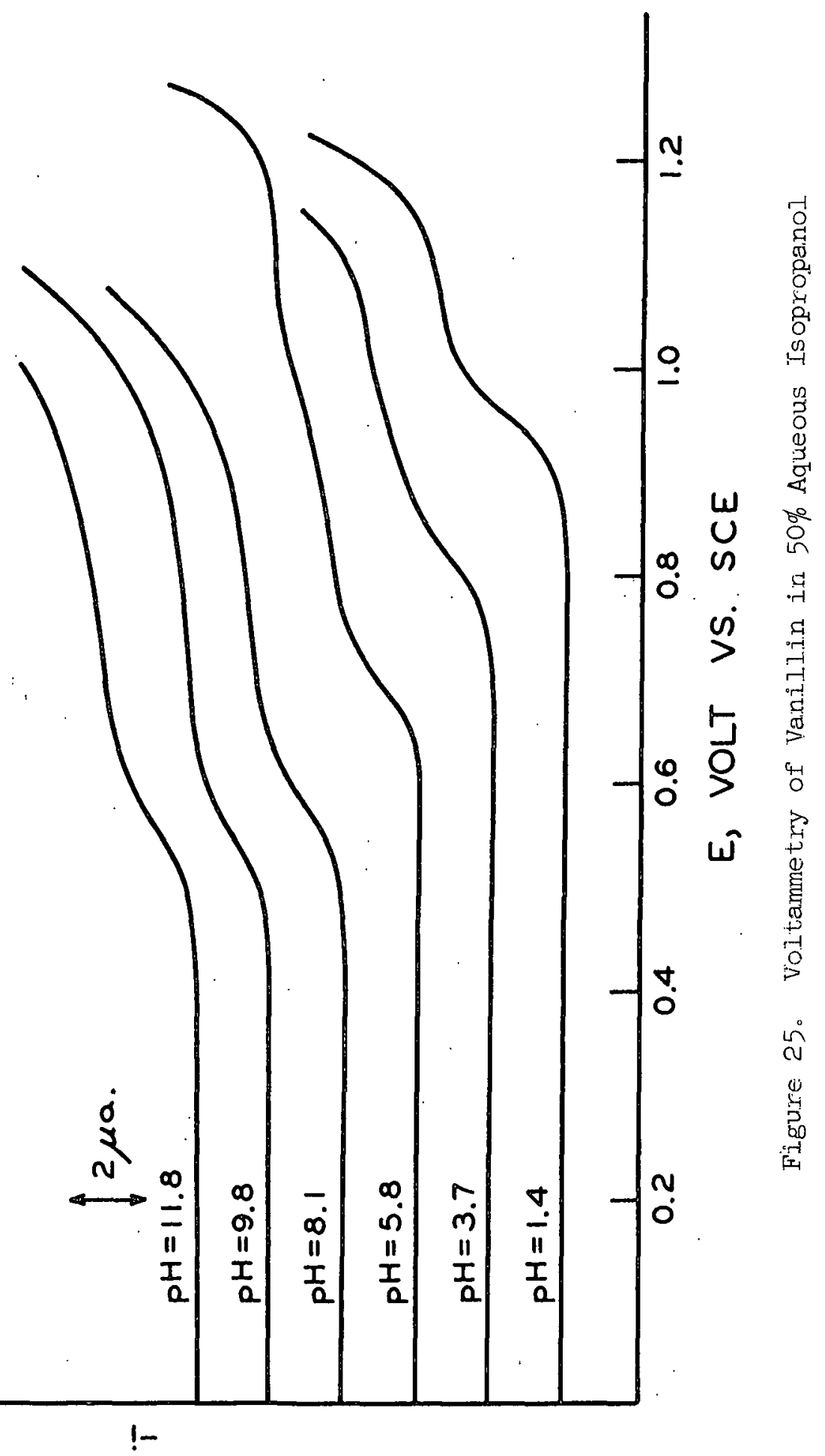


$-108-$

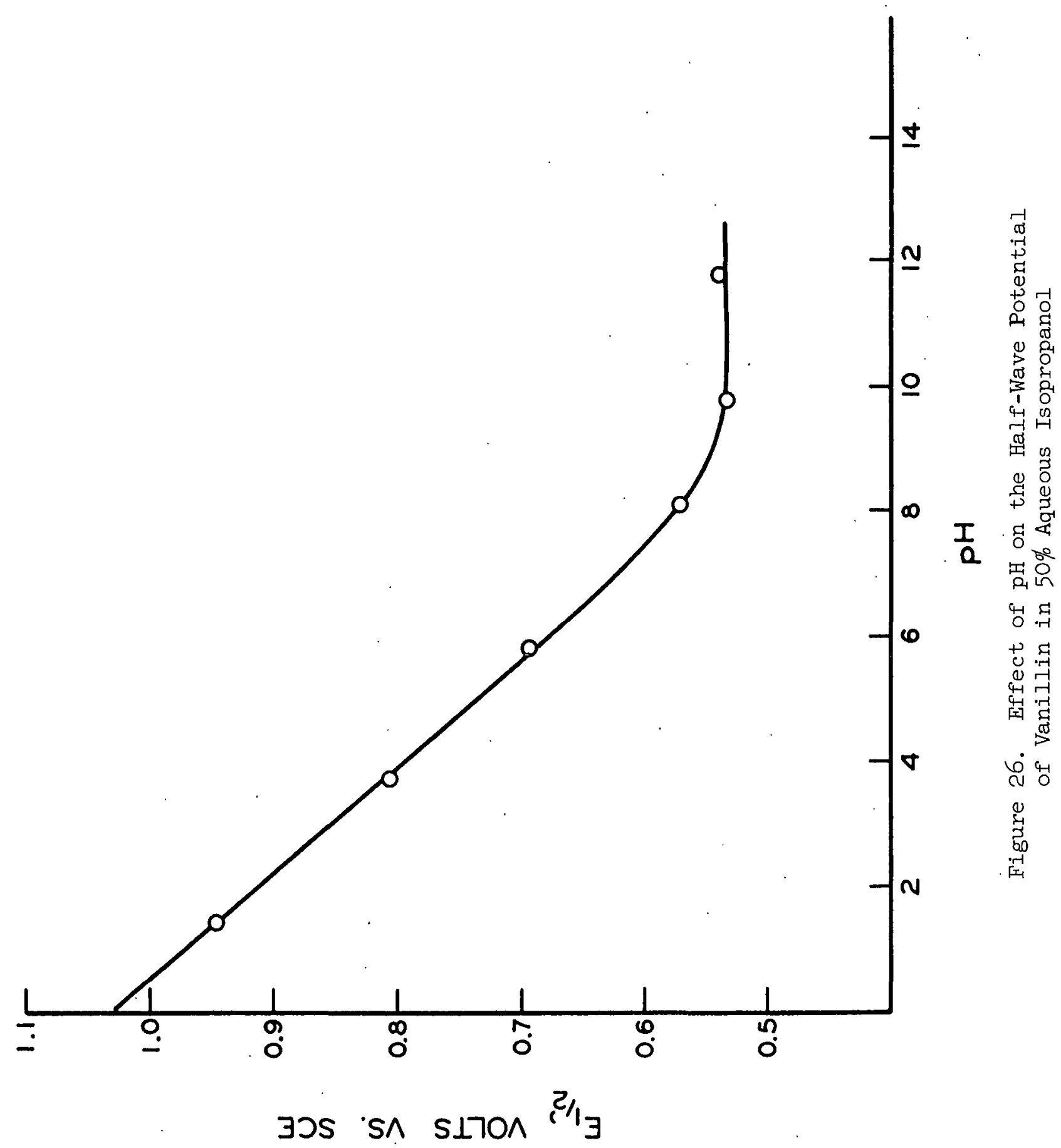




$$
\underline{E}_{0}+0.0591\left[\log \left(\underline{K} / \underline{K}_{-2}\right)+\underline{\underline{p K}}\right]=0.536+\underline{p K} \underline{\underline{D}} \cdot
$$

Since the intercept at zero $\mathrm{pH}$ is +1.028 volts, $\underline{\mathrm{pK}}$ is calculated as 8.33 . The literature value for $\mathrm{pK}$ of vanillin in water at $25^{\circ} \mathrm{C}$. is 7.396 . The difference between literature and calculated values is approximately what one would expect upon changing from water as a solvent to $50 \%$ aqueous isopropanol. Thus, $\underline{\mathrm{pK}}$ for phenol is 9.99 in water at $25^{\circ} \mathrm{C} .(\underline{76})$ and 11.28 in $49.8 \%$ ethanol at $20^{\circ} \mathrm{C}$. (77).

Using 8.33 as $\underline{\mathrm{pK}}$ for vanillin, Equation (30) fits the experimental data within a precision of 0.003 volt. This agreement of the experimental data with the theory leaves little doubt about the one-electron reversible nature of the electrode reaction of vanillin.

The limiting value of $\mathrm{E}_{1 / 2}$ at high $\mathrm{pH}$ in Fig. 26 is 0.536 volt which is about 0.31 volt more positive than $E_{1 / 2}$ for the vanillate anion in acetonitrile. This difference reflects primarily the difference between $\underline{E}_{0}$ in acetonitrile and $\underline{E}_{0}$ in water and therefore a change in the free energy for the reversible electrode reaction*.

*Changes in liquid junction potentials, hydrodynamic variables, and diffusivity are also included in the change of $\mathrm{E}_{7} / 2$ between solvents, but these are generally of a lower order of magnitude than observed here. 


\section{SUMMARY}

This investigation has shown that two entirely different anodic reactions are possible with phenolic compounds. The first is an electrophilic attack on the aromatic nucleus of the nonionized phenol with the irreversible removal of two electrons to give a mesomeric phenoxonium ion. The second is the reversible removal of one electron from the oxygen atom of the phenoxide anion to give a free radical.

In a detailed investigation of the phenoxonium ion mechanism in acetonitrile using the hindered phenol, 2,6-di-tert-butyl-p-cresol, voltammetric evidence for a dimerization reaction was obtained. It was demonstrated, however, that during an electrolysis conducted in unbuffered media, the hydrogen ion liberated at the anode would lead to a destructive oxidation of the dimeric product. Attempts at buffering the reaction mixture were unsuccessful. Therefore, a different approach to the problem of liberated hydrogen ion was indicated. An electrolysis was successfully conducted by the continuous addition of tetraethylammonium hydroxide to the anode compartment at the rate of one equivalent per Faraday of electricity. Because the tetraethylammonium hydroxide was dissolved in methanol, the dimerization reaction of 2,6-di-tert-butyl-p-cresol did not occur. Instead the principal reaction of the phenoxonium ion intermediate was the addition of methanol to form 2,6-di-tert-butyl-4-methyl-4-methoxy-2,5-cyclohexadienone in 65\% yield. This compared favorably with the theoretical yield of $71 \%$ predicted from voltammetric studies. A second product of electrolysis was 2,6-di-tert-butyl-1,4-benzoquinone in $10 \%$ yield. This product is believed to be the result of the stepwise oxidation of the para methyl group of 2,6-di-tert-butyl-p-cresol by a free radical mechanism. The free radical process occurred because a slight excess of base was present during the latter part of the electrolysis. 
Since a number of hindered phenols with different para substituents had been investigated in the study of the phenoxonium ion mechanism, the half-wave potentials were correlated by means of the Hammett sigma function. It was found that not only phenols, but also the methoxyl compounds for which Lund (31, $\underline{32}$ ) has reported data could be correlated on the same plot by considering the electrode reaction as an electrophilic attack on the aromatic nucleus.

A detailed study of the free radical reaction in acetonitrile using the vanillinate anion indicated a one-electron, reversible, primary electrode reaction. Dehydrodivanillin was obtained as the major product of electrolysis at controlled potential. It was shown by voltammetry that the monophenoxide of dehydrodivanillin would form preferentially to the vanillinate anion in acetonitrile solutions. Therefore, during the electrolysis it was necessary to add hydroxyl ion at the rate of one-half equivalent per Faraday of electricity. The yield of dehydrodivanillin was only $65 \%$, but because some unreacted vanillin remained in the solution after electrolysis it is believed that higher yields could be obtained. Since the calculated coulometric $\underline{n}$ value for this reaction was 1.77 on the basis of the recovered dehydrodivanillin as compared to the theoretical value of 1.00 , side reactions of the initial free radical were believed to have occurred.

In a Hammett-type correlation of half-wave potentials for the phenoxide anions both vanillin and the hindered phenols could be correlated on the same plot within the precision of the experimental data.

Experiments in buffered $50 \%$ isopropanol have indicated that the results of the present study are applicable to other media. Thus, either the phenoxonium ion process or the free radical process can occur depending on the $\mathrm{pH}$ of the solution and the ionization constant of the phenol. With vanillin the variation of $E_{-1 / 2}$ with 
$\mathrm{pH}$ was exactly as predicted for a fast ionization of the phenol followed by a oneelectron reversible reaction of the phenoxide anion, the mechanism originally proposed by Hedenberg and Freiser (6). From the experimental data, $\underline{\text { pK }}$ of vanillin was calculated as 8.33 in $50 \%$ aqueous isopropanol.

A voltammetric wave representing the phenoxonium ion process was observed at $\mathrm{pH}$ levels below 5.8 in 50\% aqueous isopropanol for 2,6-di-tert-butyl-p-cresol. Because the current level for this wave was abnormally low it is believed that this compound is aggregated in solution. The one-electron wave for this compound was very poorly defined at all levels of $\mathrm{pH}$ as indicated from the results of previous authors (1ㅇ, 23). 


\section{ACKNOWLEDGMENTS}

The author sincerely appreciates the advice and encouragement of his thesis committee: : Drs. I. A. Pearl, B. L. Browning, and D. G. Williams.

A special note of thanks is due Dr. J. J. O'Connor, since he conducted an electrochemical investigation simultaneously with the present study, and therefore co-operated in the solution of mutual experimental problems.

It is impossible to list each Institute staff or faculty member who has assisted in bringing this work to a successful conclusion, but the author would particularly like to express thanks to the following: Mr. E. E. Dickey for helpful discussions on many aspects of the experimental problems and results; Dr. M. A. Buchanan for advice and assistance in gas chromatographic analysis; Mr. L. G. Sel1. who ran the infrared curves and advised on their interpretation; Dr. D. C. Johnson for helpful discussions on the mechanisms of organic reactions; Mr. Keith Hardacker, and Mr. R. B. Kessler for advice on the construction of experimental equipment.

Most of all the author is indebted to his wife, Mrs. Geraldine C. Vermillion, because without her encouragement this investigation would never have been started. 


\section{LITERATURE CITED}

1. Brauns, F. E. The chemistry of lignin. New York, Academic Press, 1952. $808 \mathrm{p}$.

2. Brauns, F. E., and Brauns, D. A. The chemistry of lignin. Supplement Volume. New York, Academic Press, 1960. 804 p.

3. O'Connor, James J. The voltammetry and controlled potential electrolysis of 3,4-di-methoxypropenylbenzene. . Doctor's Dissertation. Appleton, Wisconsin, The Institute of Paper Chemistry, 1962. 100 p.

4. Allen, M. J. Organic electrode processes. New York, Reinhold Pub. Co., 1958. $174 \mathrm{p}$.

5. Mel'nikov, N. N., Sklyarenko, S. J., and Cherkasova, E..M., J. Gen. Chem. (USSR)9:1819(1939); C.A. $34: 3699$.

6. Hedenberg, J. F., and Freiser, H., Anal. Chem. 25:1355-8(1953).

7. Ginzberg, V. I., Zh. Fiz. Khim. 33:1504-15(1959)。

8. Adams, R. N., McClure, J. H., and Morris, J. B。, Anal. Chem. 30:471-7(1958).

9. Voories, J. D. Voltammetry at controlled current. The anodic oxidation of some organic compounds at solid electrodes. Doctor's Dissertation. Princeton, New Jersey, Princeton University, 1958.

10. Olsen, C., and Adams, R. N., Anal. Chim. Acta 22:582-9(1960).

11. Mueller, T. R., and Adams, R. N., Anal. Chim. Acta 23:467-79(1960).

12. Adams, R. N. Personal communication, 1961.

13. Gaylor, V. F., Elving, P. J., and Conrad, A. L., Anal. Chem. 25:1078-82(1953).

14. Gaylor, V. F., Conrad, A. L., and Ienderl, J. H., Anal. Chem. 29:224-8(1957).

15. Elving, P. J., and Krivis, A. F., Anal. Chem. 30:1645-8(1958).

16. Morris, J. E., and Schempf, J. M., Anal. Chem. 31:286-91(1959).

17. Nash, R. A., Skauen, D. M., and Purdy, W. C., J. Am. Pharm. Assoc. 47:433-5 (1958).

18. Penketh, G. E., J. Appl. Chem. (London) 7:512-2l(1957).

19. Baltes, J., Fette, Seifen, Anstrichmittel 56:484-90(1954); C.A. 50:3117.

20. Suatoni, J. C., Snyder,.R..E., and Clark, R。 O., Anal. Chem. 33:1894-8(1961).

21. Gould, N. S. Mechanism and structure in organic chemistry. New York, Holt, Rinehart, and Winston, 1959. $790 \mathrm{p}$. 
22. Elving, F. J., and Krivis, A. F., Anal. Chem. 30:1648-52(1958).

23. Barendrecht, E., Anal. Chim. Acta 24:498-9(1961).

24. Pearl, I. A., J. Am. Chem. Soc. 74:4260-2(1952).

25. Lund, Henning. Personal Communication, 1961.

26. Geske, D. H., J. Electroanal. Chem. 1:502-3(1960).

27. Geske, D. H. Personal communication, 1961.

28. Geske, D. H. Voltammetric studies in acetonitrile. Doctor's Dissertation. Iowa City, Iowa, State University of Iowa, 1957. 262 p.

29. Geske, D. H., J. Am. Chem. Soc. 81:4145-7(1959).

30. Geske, D. H., J. Phys. Chem. 63:1062-70(1959).

31. Luna, Henning, Acta Chem. Scand. 11:491-8(1957).

32. Lund, Henning, Acta Chem. Scand. 11:1323-30(1957).

33. Kolthoff, I. M., and Coetzee, J. F., J. Am. Chem. Soc. 79:870-4(1957).

34. Kolthoff, I. M., and Coetzee, J. F., J. Am. Chem. Soc. 79:1852-8(1957).

35. Coetzee, J. F., and Kolthoff, I. M., J. Am. Chem. Soc. 79:1610-15(1957).

36. Kolthoff, I. M., Bruckenstein, S., and Chantooni, M..K., Jr., J. Am. Chem. Soc. 83:3927-35(1961).

37. Kolthoff, I. M. , and Lingane, J. J. Polarography. 2d. ed. New York, Interscience, 1952. $990 \mathrm{p}$.

38. Licht, T. S., Curran, D. J., and de Bethune, A. J., Anal. Chem. 30, no. $11: 1888(1958)$.

39. Hume, D. N., Anal. Chem. 34, no. 5:172R-182R(1962).

40. Meites, Lewis. Polarographic techniques. New York, Interscience, 1955. $317 \mathrm{p}$.

41. Manuals of instruction for the Analytical Instruments Inc. Potentiostat and Current Integrator. Walcott, Conn. Analytical Instruments Inc., 1961. 23 p.

42. Delahay, Paul. New instrumental methods in electrochemistry. New York, Interscience, 1954. $437 \mathrm{p}$.

43. Wawzonek, S., and Runner,. M. E., J. Electrochem. Soc. 99:457(1952).

44. Burdick, .D. L., Santoro, A. V., McEwen, W. E., and Kleinberg, J., J. Am. Chem. Soc. 79:5467-70(1957). 
45. Boedecker, F., and Volk, H., Ber. 64:61-6(1931).

46. Cook, C. D., J. Org. Chem. 18:261-6(1953).

47. Moore, R. F., and Waters, W. A., J. Chem. Soc. 1955:2753-5.

48. Coppinger, G., and Campbell, T., J. Am. Chem. Soc. 75:734-6(1953).

49. Cook, C. D., English, .E. S., and Wilson, B. J., J. Org. Chem. 23:755-6(1958).

50. Yohe, G. R., Dunbar, J. E., Pedrotti, R. L., Scheidt, F. M. , Lee, F. G., and Smith, E. C., J. Org. Chem. 21:1289-92(1956).

51. Young, L. Anodic oxide films. New York, Academic Press, 1961. 377 p.

52. Kolthoff, I..M., and Tanaka, N., Anal. Chem. 26:632-6(1956).

53. Anson, F. C., and Lingane, J. J., J. Am. Chem。 Soc. 79:4901-4(1957).

54. Laitinen, H. A., and Enka, C. G., J. Electrochem. Soc. 107:773-81(1960).

55. Anson, F. C., Anal. Chem。33:934-9(1961).

56. Lingane, J. J., J. Electroanal. Chem. 1:379(1960).

57. Bickel, A. F., and Kooyman, E. G., J. Chem. Soc. 1953:3211-18.

58. Bellamy, L. J. The infrared spectra of complex molecules. New York, John Wiley and Sons, Inc., 1956. $323 \mathrm{p}$.

59. Dickey, .E. E. Personal communication, 1961.

60. Potter, E. C. Electrochemistry. London, Cleaver-Hume Press, Ltd., 1956. $4.18 \mathrm{p}$.

61. Larson, R. C., Iwamoto, R. T., and Adams, R. N., Reference electrodes for voltammetry in acetonitrile. A preprint supplied by Dr. R. N. Adams, 1961.

62. Eisenberg, M., Tobias, C. W., and Wilkie, C. R., J. Electrochem. Soc. 101: 306-20(1954).

63. Nightingale, E. R。, Jr。, Anal. Chim。 Acta 16:493-6(1957).

64. Fujisaki, T., Nippon Kagaku Zasshi 77:727-31(1956); C.A. $52: 8096$.

65. Fujisaki, T., Nippon Kagaku Zasshi 77:731-2; C.A. 52:8096-7.

66. Fujisaki, T., Nippon Kagaku Zasshi 77:733-4; C.A. 52:8097.

67. Fujisaki, T., Nippon Kagaku Zasshi 77:869-73; C.A. 52:8097.

68. Kharasch, .M. S., and Joshi, B. So, J. Org. Chem. $22: 1439-44$ (1957). 
69. Cook, C. D., and Woodworth, R. C., J. Am. Chem. Soc. 75:6242-4(1953).

70. Jaffe, H. H., Chem. Rev. 53:191-262(1953).

71. Pearl, I. A., J. Org. Chem. 12:85-9(1947).

72. Thyagarajan, B. S., Chem. Rev. 58:439-60(1958).

73. Barton, D. H. R., and Cohen, T., Festschr. Arthur. Stol1. 1957:117-43.

74. Erdtman, H., and Wachtmeister, C. A., Festschr. Arthur Stoll. 1957:144-65.

75. Elving, . ...J., Markowitz, J..M., and Rosenthal, I., Anal. Chem. 28:1179-80 (1956).

76. Kortum, G., Vogel, W., and Andrussow, K. Dissociation constants of organic acids in aqueous solutions. London, $1961.450 \mathrm{p}$.

77. Schwarzenback, G., and Rudin, E., Helv. Chim. Acta 22:360-76(1939). 


\section{APPENDIX}

FURTHER EXPERTMENTS WITH PHENOLS IN ACETONITRILE

\section{2,6-DIMETHOXYPHENOL}

This compound was notable because it was the only phenol investigated which gave a diffusion current that would indicate a four-electron reaction. Thu: in the concentration range from 0.0258 to $0.103 \mathrm{mM}$ (electrode No. 8, oxidized; 0.1M tetraethylammonium perchlorate) $\underline{i} \underline{\underline{D}} \underline{\mathrm{C}}$ was -74 to $-81 \mu \mathrm{a} . / \mathrm{mM}$ which is about twice the level of $\underline{I}_{\underline{D}} / \underline{C}$ in Table VI on the same electrode. The half-wave potential was 1.08 volts with $\beta \underline{n}_{\beta}$ ranging from 0.61 to 0.72 .

It seems very improbable that the electrode reaction of 2,6-dimethoxyphenol involves the simultaneous transfer of four electrons. More logically the primary process involves two electrons as with the other phenols, but this is followed by a fast rearrangement, and a second two-electron transfer.

When $\sigma^{+}$values are calculated for the meta and para positions to the phenolic hydroxyl for this compound, it is found that the most negative $\sigma^{+}$value is obtained at the meta position. In other words, the two methoxyl groups both contribute significantly to the increased electron density in this position, and this should be the point of the electrode attack. In agreement with this reasoning, the point for :2,6-dimethoxyphenol in Fig. $17 \mathrm{falls}$ on the same line with the other compounds.

One possibility which would explain the high value of $\underline{i}_{\underline{D}} / \underline{C}$ for 2,6-dimethoxyphenol is a demethylation followed by a further electrode reaction and ring cleavage. The present data are, however, too limited to warrant drawing any definite conclusion. 


\section{ISOEUGENOL}

The data in Table IX were taken on Electrode No. 7 with an oxidized surface. The supporting electrolyte was $0.1 \mathrm{M}$ sodium perchlorate. At concentration lievels of $0.2 \underline{m M}$ and lower the value of $\underline{i}_{\underline{D}} / \underline{C}$ for isoeugenol in Table IX represents a twoelectron transfer by comparison with hydroquinone. At.higher levels of concentration a sharp decrease of $-\underline{i} \underline{D} \underline{C}$ is noted in Table IX. Furthermore, it was not possible to retrace a voltammogram even at very low concentrations without cleaning the electrode with hot chromic acid. This behavior is typical of film-forming phenols $(\underline{6}-\underline{8})$.

\section{TABLE IX}

VOLTAMMETRY OF ISOEUGENOL ${ }^{\mathrm{a}}$

(Electrode No. 7, oxidized surface; $0.1 \mathrm{M}$ sodium perchlorate; rate of polarization $=2.2 \overline{\mathrm{mv}} \cdot / \mathrm{sec}$.

\begin{tabular}{|c|c|c|c|}
\hline $\begin{array}{c}\text { Concn., } \\
\underline{\mathrm{mM}}\end{array}$ & $\begin{array}{l}-\underline{\underline{i}}-\underline{\mathrm{C}}, \\
\mu \mathrm{a} . / \mathrm{mM}\end{array}$ & $\begin{array}{l}E_{1 / 2}, \\
\text { volts }\end{array}$ & $\beta \stackrel{n}{n}_{\beta}$ \\
\hline 0.0235 & 32.7 & 0.90 & 0.64 \\
\hline 0.0470 & 37.2 & 0.94 & 0.47 \\
\hline 0.0705 & 34.9 & 0.94 & 0.51 \\
\hline 0.0940 & 39.7 & 0.96 & 0.44 \\
\hline 0.118 & 38.7 & 0.98 & 0.34 \\
\hline 0.188 & 30.1 & 0.96 & 0.41 \\
\hline 0.470 & 27.6 & 0.96 & 0.45 \\
\hline 0.940 & 17.6 & 0.97 & 0.5 \\
\hline
\end{tabular}

${ }^{a}$ Cell resistances for this data were measured with the Leeds \&. Northrup bridge. Therefore, an error in. $E_{-1 / 2}$ and $\beta \underline{n}_{-\beta}$ may have resulted as noted in the experimental section. 
EFFECT OF PYRIDINE

With the nonhindered phenols, pyridine is a strong enough base to cause partial ionization of the phenolic hydroxyl, and this results in a prewave which precedes the main two-electron wave in unbuffered acetonitrile. This behavior has been noted previously by Lund (25), and was observed in the present study for isoeugenol and vanillin.- Picric acid gives a nicely formed, apparently reversible one-electron wave at $1.49-1.50$ volts on an oxidized electrode at concentration: levels from 0.1 to $1 \mathrm{mM}$ in the presence of equivalent pyridine.

Hydroquinone in $0.1 \mathrm{M}$ pyridine on an oxidized electrode displays a considerably less positive half-wave potential than in the absence of pyridine. However, $E_{1 / 2}$ is strongly dependent upon concentration, varying from 0.47 volt at $0.06 \mathrm{mM}$ to 0.59 volt at $1.2 \mathrm{mM}$, an effect not observed in the absence of pyridine. This behavior is characteristic of a "reversible" electrode process in an unbuffered solution when hydrogen ions are liberated at the electrode; yet the $\beta \underline{n}_{\beta}$ value of 0.3 to 0.4 indicates a high degree of irreversibility. The value of $\underline{i}_{\underline{D}} / \mathrm{C}$ is approximately the same as is observed without added pyridine. It seems likely from these observations that the reaction mechanism in this case is not the carbonium ion process, but rather involves a free radical reaction of the nonionized form of hydroquinone to give transient semiquinone formation with rapid stabilization and reoxidation.

In the presence of an equivalent concentration of pyridine, 2,6-DTBC at concentration levels from 0.1 to $1 \mathrm{mM}$ behaves in much the same manner as in the absence of pyridine, a two-electron process being indicated. The main difference with the addition of an equivalent amount of pyridine is that the half-wave potential is shifted to a more positive value, and $\beta \underline{n}_{\beta}$ for the reaction is 
decreased. This effect is particularly pronounced on oxidized electrodes as depicted in Fig. 27. The shift to a higher potential in the presence of equivalent pyridine is not explainable on the basis of any inductive effects the base might have on the phenolic molecule. These effects would instead be expected to lower the half-wave potential in the manner of added water or methanol. No other explanation for this behavior can be advanced at the present time unless it reflects a change in the condition of the electrode surface in the presence of pyridine. It seems likely that the pyridine at these low concentration levels functions mainly as a proton acceptor as noted by Lund (3I) in the oxidation of phenylcarbinols.

With an increase in the pyridine concentration to $0.1 \mathrm{M}, 2,6-\mathrm{DTBC}$ and the other phenols studied: give the peculiar peaked waves shown in Fig. 28. Vanillin and picric acid are not shown in the figure, but are also known to give peaks in the same potential range as the compounds of Fig. 28. The occurrence of peaked waves in the presence of pyridine has been reported previously by Geske (28) in the oxidation of halogen species, and by O'Connor (3) for 3,4-dimethoxypropenyl. benzene, but in these cases the extremely rapid fall-off of current to nearly zero as indicated in Fig. 28 was not observed. Several points of particular interest were noted for the phenols.

1. The peaks occurred in a narrow range of potential for all the phenols studied ( 1.55 to 1.70 volts) despite the wide range of half-wave potentials for these same phenols in the absence of pyridine. (1.08 Volts for 2,6-dimethoxyphenol to $>2.0$ volts for picric acid.) Furthermore, although the peak height was considerably lower on an oxidized than a reduced electrode, the condition of the electrode surface had no significant effect on the peak potential. This behavior indicates that a normal electrode reaction occurs up to a certain potential, where a new reaction results in a film formation on the electrode surface; and that the same reaction is responsible for the peaks with each compound. 


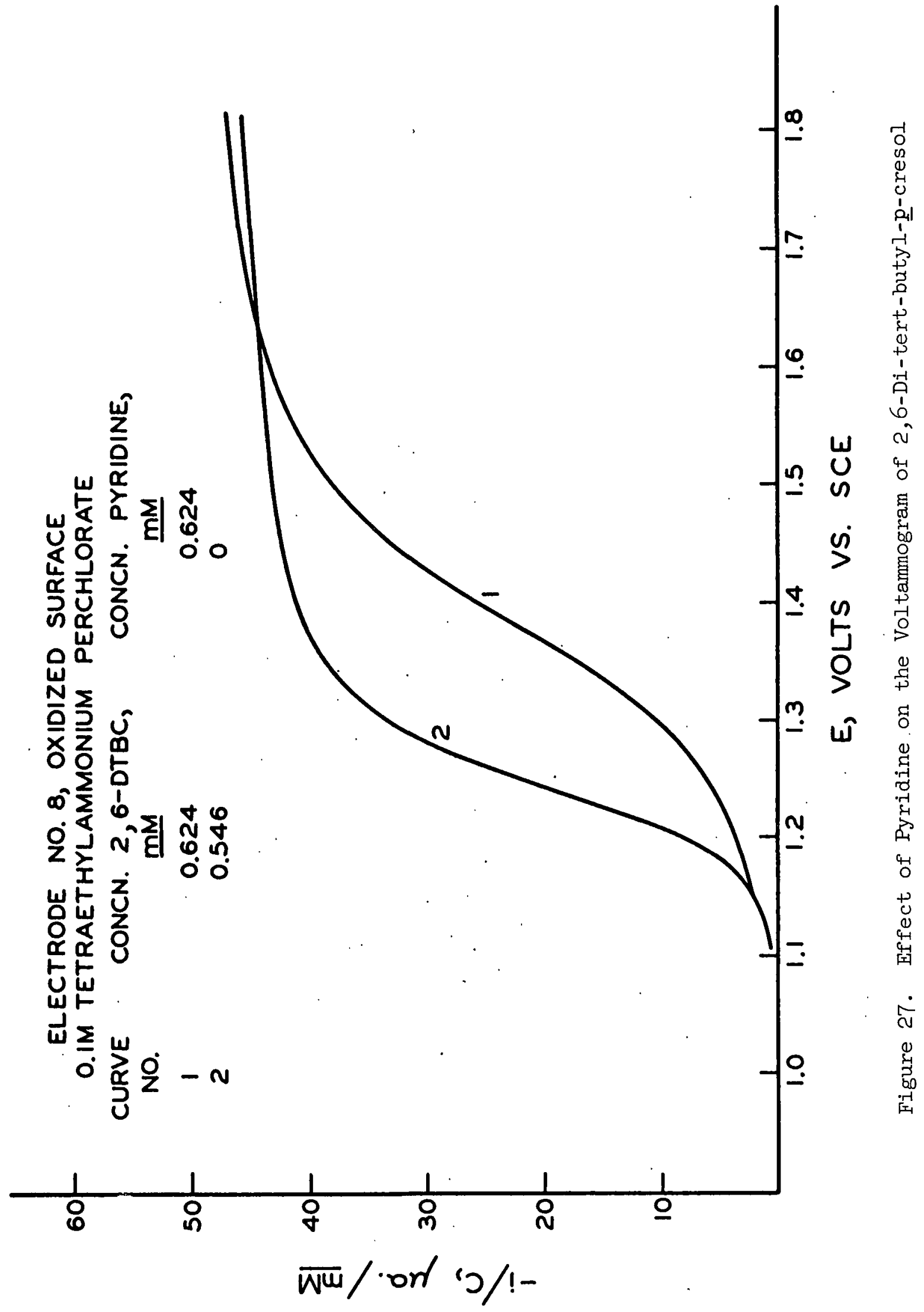




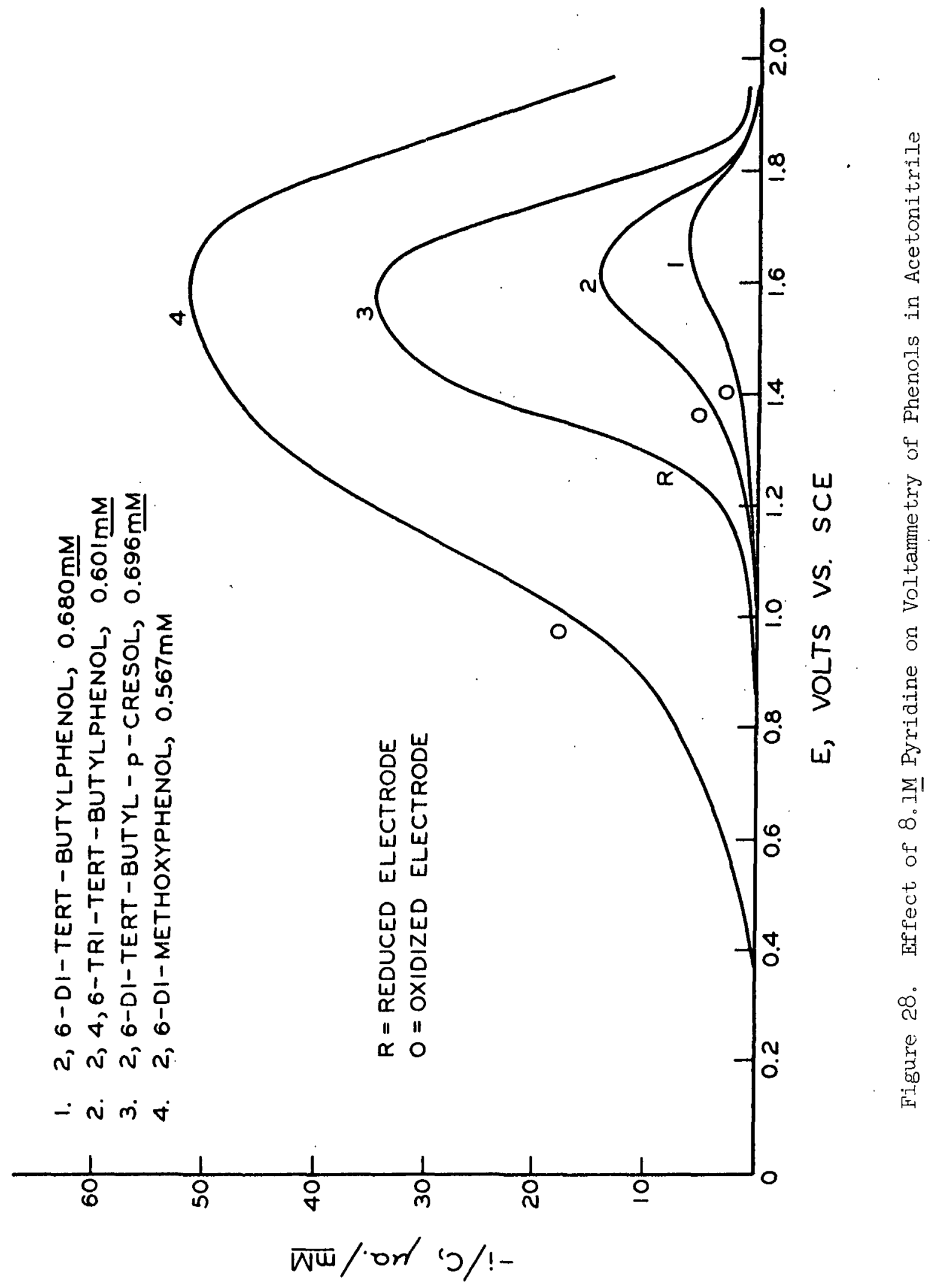


2. Practically no current flows in retracing the voltammogram in the opposite direction (+ to -), again indicating a surface film on the electrode.

3. The film is evidently not polymeric in nature because the voltammogram can be retraced perfectly after cathodic treatment of the electrode. Also, the peaks occur even at extremely low levels of concentration (0.022mM) where one would expect that, if a polymerization were occurring, the rate of covering of the electrode surface would be reduced.

A possible explanation for this behavior is that the platinum surface atoms of the electrode form some type of insoluble complex with the products of oxidation, and that this complex effectively blocks the surface for the further transfer of electrons. Such a complex might be associated with a definite change in the oxidation state of the surface platinum atoms--hence, the very rapid drop in current level.

Nothing definite can be said regarding the nature of such a surface complex from the results of this study. The first possibility that comes to mind is that the mesomeric phenoxonium ion from the two-electron oxidation of a phenolic molecule might be stabilized by addition of pyridine to form a pyridinium salt which then complexes with the platinum. There is a precedent for the obtaining of pyridinium cations from electrochemical oxidations in acetonitrile in the results of Lund with anthracene (32) and $0^{\prime}$ Connor with 3,5-dimethoxypropenylbenzene (3). However; in the case of 2,4,6-tri-tert-butylphenol, steric considerations make such a possibility unlikely. Furthermore, the extremely high peak current obtained with picric acid ( $-112 \mu \mathrm{a} . / \mathrm{mM}$ on electrode No. 8) cannot be explained on this basis. The peak height of picric acid would represent approximately a five-electron transfer by comparison with the results of Table VI. Yet, it seems likely that a reaction of the picrate anion is being observed, since in the absence of pyridine, 
picric acid is voltammetrically inactive, and with the addition of equivalent pyridine an apparently reversible one-electron wave is observed.

THE USE OF HAMMETT CORRELATIONS WITH HALF-WAVE POTENTIALS

\section{TOTALLY IRREVERSIBLE ELECTRODE REACTION}

Another form of Equation (5). for a totally irreversible reaction at $25^{\circ} \mathrm{C}$. is

$$
E_{i / 2}=-\frac{0.0591}{\beta n_{B}}\left[\log k_{b, h}^{\circ}+\log \left(\delta_{R} / D_{R}\right)\right]
$$

The Hammett relationship (Iㅡ) states that the rate constant for a phenol with any substituent, $\underline{\mathrm{k}}_{\underline{\mathrm{b}}}^{\mathrm{O}}, \underline{\mathrm{h}}$, is related to the rate constant for a hydrogen substituent, $\left(\underline{k}^{\circ} \underline{\mathrm{b}}, \underline{\mathrm{h}}\right)^{\circ}$, by Equation $(36)$, where $\sigma$, and $\rho$ are the Hammett substituent and reaction constants, respectively.

$$
\log \left[k_{b, h}^{0} /\left(k_{b, h}^{\circ}\right)^{0}\right]=\sigma \rho
$$

From Equation (35) the relationship between the half-wave potential for a phenol with any substituent, $\underline{E}_{1 / 2}$, and the half-wave potential with a hydrogen substituent, $E_{1 / 2}^{0}$, is

$$
E_{l / 2}-E_{l / 2}^{0}=-\frac{0.0591}{\beta n_{B}}\left[\log \frac{k_{b, h}^{0}}{\left(k_{b, h}^{0}\right)^{0}}+\log \frac{\delta_{R} / D_{R}}{\delta_{R}^{0} / D_{R}^{0}}\right]
$$

Assuming that $\log \delta_{\underline{R}} \underline{D}_{\underline{R}}^{\circ} / \underline{S}_{\underline{R}}^{\circ} \underline{D_{R}}$ is essentially zero, a combination of Equations (36) and (37) gives the required relationship, Equation (19).

$$
E_{1 / 2}-E_{I / 2}^{0}=-\frac{0.0591}{\beta n_{B}} \sigma \rho
$$




\section{REVERSIBLE REACTION}

For a reversible one-electron reaction in which hydrogen ions are not involved, Equation (3) becomes at $25^{\circ} \mathrm{C}$.

$$
E_{I / 2}=E_{0}-0.0591 \log \left[\frac{D_{0} \delta_{R} f_{R}}{D_{R} \delta_{0} f_{0}}\right] \text {, }
$$

but by definition

$$
E_{0}=-0.0591 \log (1 / K)
$$

where $\underline{K}$ is the equilibrium constant for the electrode reaction when written as an oxidation. Therefore

$$
E_{I / 2}=-0.0591\left(\log I / K-\log \frac{D_{0} \delta_{R} f_{R}}{D_{R} \delta_{0} f_{0}}\right)
$$

The Harmett equation for an equilibrium reaction is stated as

$$
\log \mathrm{K} / \mathrm{K}_{0}=\sigma \rho
$$

where $\underline{K}$ is the equilibrium constant for a phenol with any substituent, and $\underline{K}_{\underline{O}}$ is the equilibrium constant for a phenol with a hydrogen substituent. By combining Equations (39) and (40) in the same manner as for the irreversible case and making the same assumption, the required expression, Equation (28), is obtained.

$$
\underline{E}_{1 / 2}-\underline{E}_{1 / 2}^{0}=-0.0591 \sigma \rho
$$

REVERS IBLE REACTION OF PHENOLS, BUFFERED MEDIA

For a $\mathrm{pH}$ high enough so that $\underline{\underline{K}} \underset{\underline{D}}{\mathrm{P}} \gg\left[\mathrm{H}^{+}\right]$the case is identical to that previously discussed, and Equation (28) applies. 
At a $\mathrm{pH}$ low enough so that $\left[\mathrm{H}^{+}\right] \gg \underline{-}_{-}$the expression for $\underline{E}_{-1 / 2}$ is given by Equation (33),

$$
E_{1 / 2}=E_{0}+0.0591\left(\log K_{1} / K_{2}-p H+p K_{D}\right) .
$$

Here $\underline{E}_{\underline{O}}$ is again given by Equation 38), and the Hammett relationship of Equation (40) applies. The dissociation constant of the phenol can also be expressed in a Hammett-type expression,

$$
\log \mathrm{K}_{\mathrm{D}} / \mathrm{K}_{\mathrm{D}}^{\circ}=\sigma \rho^{\prime}
$$

where $\underline{K}_{\mathbb{D}}$ is the dissociation constant for any phenol, $\underline{K}^{0}$ is the dissociation constant for phenol, and $\rho^{\prime}$ is the Hammett reaction constant for the dissociation constants of phenols. By combining Equations (33), (40), and (41) the required expression is Equation (34).

$$
E_{1 / 2}-E_{1 / 2}^{O}=-0.0591 \sigma\left(\rho+\rho^{\prime}\right)
$$

